Florida International University FIU Digital Commons

2-12-1996

\title{
A New Microprocessor-Controlled Stimulator for Visual Evoked Potential Acquisition
}

Octavio Gabriel Garrastacho

Florida International University

DOI: $10.25148 /$ etd.FI15101539

Follow this and additional works at: https://digitalcommons.fiu.edu/etd

Part of the Electrical and Computer Engineering Commons

\section{Recommended Citation}

Garrastacho, Octavio Gabriel, "A New Microprocessor-Controlled Stimulator for Visual Evoked Potential Acquisition" (1996). FIU Electronic Theses and Dissertations. 3592.

https://digitalcommons.fiu.edu/etd/3592

This work is brought to you for free and open access by the University Graduate School at FIU Digital Commons. It has been accepted for inclusion in FIU Electronic Theses and Dissertations by an authorized administrator of FIU Digital Commons. For more information, please contact dcc@fiu.edu. 


\title{
FLORIDA INTERNATIONAL UNIVERSITY
}

\author{
Miami, Florida
}

A New Microprocessor-Controlled Stimulator for Visual Evoked

Potential Acquisition

A thesis submitted in partial satisfaction of the

requirements for the degree of

MASTER OF SCIENCE

IN

ELECTRICAL ENGINEERING

by

Octavio Gabriel Garrastacho

1996 
To: College of Engineering and Design (Dr. Gordon R. Hopkins, Dean)

This thesis, written by Octavio Gabriel Garrastacho, and entitled A new Microprocessor-Controlled for Visual Evoked Potential Acquisition, having been approved in respect to style and intellectual content, is referred to you for judgment.

We have read this thesis and recommend that it be approved.

Dr. Malcom Heimer

Dr. Özcan Özdamar

Dr. Armado Barreto, Major Professor

Date of Defense: February 12, 1996

The thesis of Octavio Gabriel Garrastacho is approved.

Dr. Gordon R. Hopkins

Dean of College of Engineering and Design

Dr. Richard C. Campbell

Dean of Graduate Studies

Florida International University, 1996 
I dedicate this thesis to my wife and my parents, especially my father. He taught me never to quit seeking my aspiration in life. Without his understanding, support and friendship, the completion of this work would not have been possible.

Que Dios este contigo, mi viejo. 


\section{ACKNOWLEDGMENTS}

I wish to thank the members of my committee for their helpful comments and patience. I also want to thank the members of Intelligent Hearing Systems Corporation: Dr. Rafael Delgado, for his help in everything; Carlos Lopez, for timely ideas and encouraging comments; Isiari Morales and Syed Raman for their assistance.

A special thanks must go to my major professor, Dr. Barreto, for his support and encouraging comments, and especially for having patience and confidence in me, I am also thanking to the other members of my committee, Dr. Özdamar and Dr. Heimer for their assistance. 


\author{
ABSTRACT OF THE THESIS
}

\title{
A New Microprocessor-Controlled Stimulator for Visual Evoked Potential Acquisition
}

\author{
by \\ Octavio Gabriel Garrastacho
}

Florida International University, 1996

\section{Professor Armando B. Barreto, Major Professor}

The goal of this study was to develop two computer-controlled visual evoked potential (VEP) stimulators. The first device employs a $12 \times 12$ matrix of $5 \mathrm{~mm}$ square Light Emitting Diodes (LEDs) and is housed in an $8 \times 8$ utility box structure. The second device employs two $8 \times 8$ matrices of $3 \mathrm{~mm}$ square LEDs, each housed in one eyepiece of a goggle-like structure. A quantitative comparison of the performance of these stimulators was carried out in terms of absolute and interpeak latencies, signal-to-noise ratio (SNR) and cross-correlation between sequential responses obtained from them. Six normal adult subjects were involved in the comparison. Data were acquired from monocular full-field stimulation. The comparison emphasizes potential advantages of the newer, gogglemounted stimulator. 


\section{TABLE OF CONTENTS}

CHAPTER

PAGE

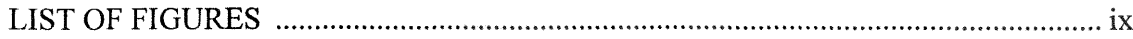

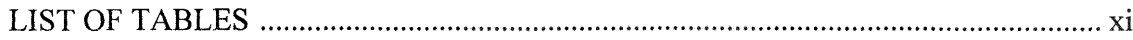

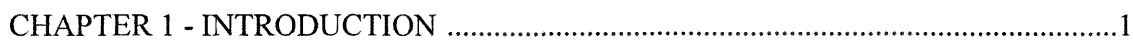

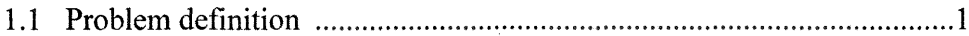

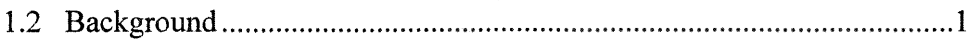

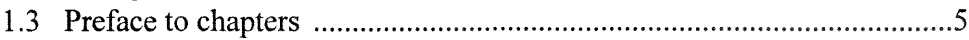

CHAPTER 2 - ELECTROPHYSIOLOGY AND VEP CHARACTERISTICS ................. 7

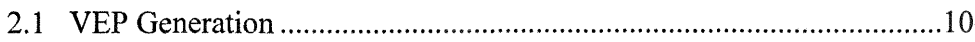

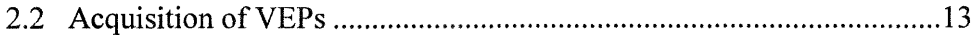

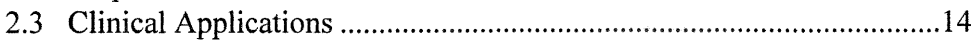

CHAPTER 3 - PATTERN CHECKERBOARD REVERSAL STIMULATORS .............16

3.1 Television screen pattern stimulator ......................................................16

3.2 Slide-projector stimulator ...............................................................16

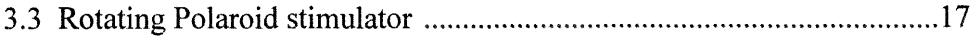

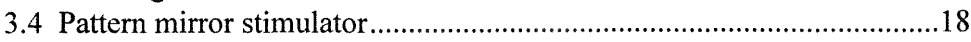

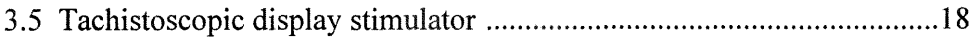

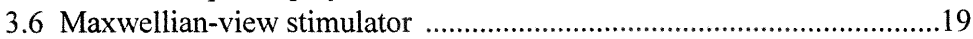

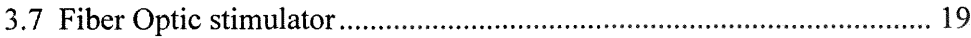

3.8 Light-emitting diodes matrix display stimulator .................................20

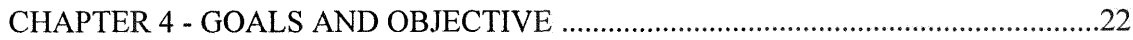

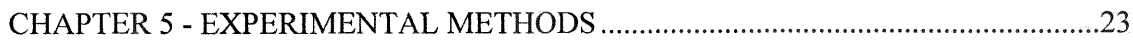

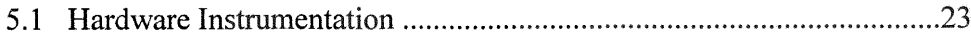

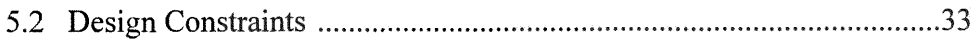

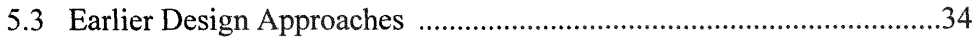

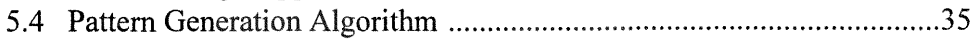

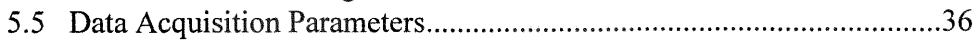

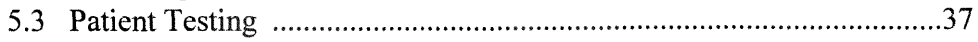




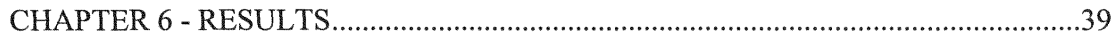

6.1 Latency-interpeak amplitude Analysis....................................................

6.2 Observations on the results and comparison with other data..................75

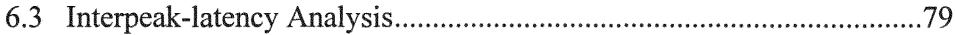

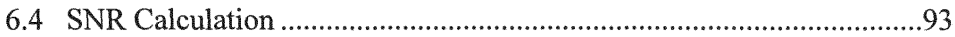

6.5 Cross Correlation Calculation..............................................................104

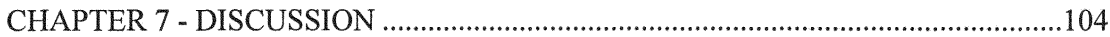

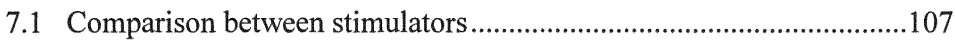

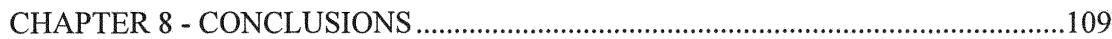

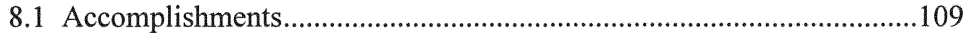

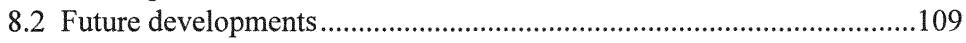

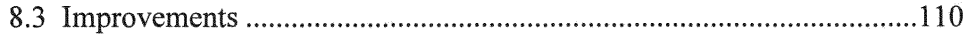

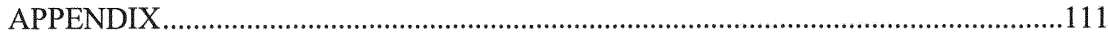

A.1 Calculation of size of the checks........................................................111

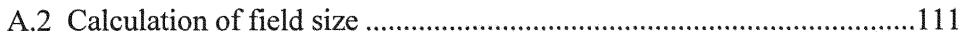

A.3 Calculation of the Stimulus Luminance ................................................112

A.4 Calculation of the Contrast .................................................................112

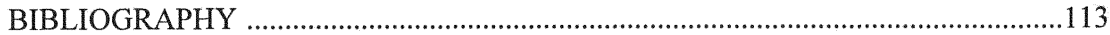




\section{LIST OF FIGURES}

PAGE

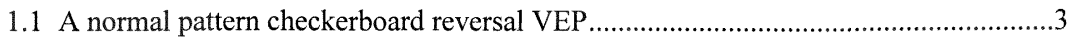

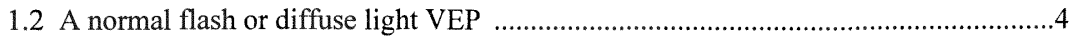

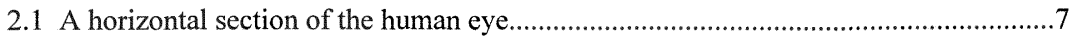

2.2 The primary visual pathway of a human eye ............................................................

2.3 Diagrammatic Representation of the positions and orientations of VEP dipoles.....12

5.1 Schematic diagram of the visual evoked potential system …….................................25

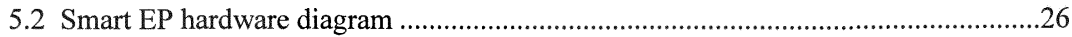

5.3a Block diagram of the data acquisition and timing for Smart-EP VEP .......................28

5.3b Flow diagram of the Set Inter Stimulus Interval function ........................................29

5.4 A Block diagram of the Visual Box stimulator ...........................................................

5.5 A Block diagram of the Visual Goggle stimulator .....................................................33

5.6a Flow diagram of the pattern generation program.....................................................35

$5.6 \mathrm{~b}$ Screen display of the VEP's pattern reversal generation program .............................35

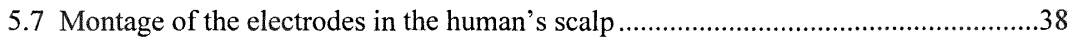

6.1 How to measure peak latency, interpeak latency and amplitude of a VEP.................42

6.2 Two superimposed recordings obtained from both stimulators..................................44

6.3a Mean latencies in four different ranges of sweeps - first recording, subject CL.......46

$6.3 \mathrm{~b}$ Interpeak amplitudes in four different ranges of sweeps - first recording, subject $\mathrm{CL}$

6.3c Mean latencies in four different ranges of sweeps - second recording, subject CL..48 $6.3 \mathrm{~d}$ Interpeak amplitudes in four different ranges of sweeps - second recording, subject CL

6.4a Mean latencies in four different ranges of sweeps - first recording, subject CQ ......51 $6.4 \mathrm{~b}$ Interpeak amplitudes in four different ranges of sweeps - first recording, subject CQ

6.4c Mean latencies in four different ranges of sweeps - second recording, subject CQ .53 
6.4d Interpeak amplitudes in four different ranges of sweeps - second recording, subject CQ.

6.5a Mean latencies in four different ranges of sweeps - first recording, subject IM.......56

$6.5 \mathrm{~b}$ Interpeak amplitudes in four different ranges of sweeps - first recording, subject IM

6.5c Mean latencies in four different ranges of sweeps - first recording, subject IM.......58

6.5d Interpeak amplitudes in four different ranges of sweeps - first recording, subject IM

6.6a Mean latencies in four different ranges of sweeps - first recording, subject LO ......61

6.6b Interpeak amplitudes in four different ranges of sweeps - first recording, subject LO.....

6.6c Mean latencies in four different ranges of sweeps - first recording, subject LO ......63

$6.6 \mathrm{~d}$ Interpeak amplitudes in four different ranges of sweeps - first recording, subject LO.

6.7a Mean latencies in four different ranges of sweeps - second recording, subject MS

6.7b Interpeak amplitudes in four different ranges of sweeps - second recording, subject MS

6.7c Mean latencies in four different ranges of sweeps - first recording, subject MS ......68

$6.7 \mathrm{~d}$ Interpeak amplitudes in four different ranges of sweeps - first recording, subject MS

6.8a Mean latencies in four different ranges of sweeps - first recording, subject RD ......71

$6.8 \mathrm{~b}$ Interpeak amplitudes in four different ranges of sweeps - first recording, subject RD.

6.8c Mean latencies in four different ranges of sweeps - second recording, subject RD .73 $6.8 \mathrm{~d}$ Interpeak amplitudes in four different ranges of sweeps - second recording, subject RD .74

6.9a Comparison of normal mean latencies versus thesis's mean latencies ......................78

6.9b Comparison of normal mean amplitudes versus thesis's mean amplitudes...............78

6.10 A graphical representation of the interpeak latencies obtained from both stimulators versus the normal latencies .92

6.11 SNR Analysis Algorithm Flow Diagram .98 
6.12 Pre and post stimulus SNR estimate of a recording acquired with VEP-Box .99

6.13 Pre and post stimulus SNR estimate of a recording acquired with VEP-Goggle ...100 6.14 SNR calculations and its average VEP response - Subject 2 ................................101

6.15 SNR calculations and its average VEP response - Subject 4 …............................102

6.16 SNR calculations and its average VEP response - Subject 6 ................................103

6.17 Cross Correlation results from right and left eyes obtained from both stimulators 


\section{LIST OF TABLES}

PAGE

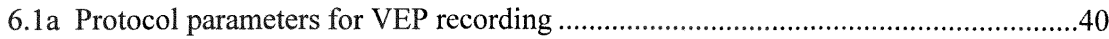

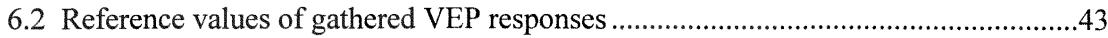

6.3a Mean latencies and interpeak amplitudes of VEPs from both eyes of subject CL's first recording using both stimulators

6.3b Mean latencies and interpeak amplitudes of VEPs from both eyes of subject CL's second recording using both stimulators.

6.4a Mean latencies and interpeak amplitudes of VEPs from both eyes of subject CQ's first recording using both stimulators

6.4b Mean latencies and interpeak amplitudes of VEPs from both eyes of subject CQ's second recording using both stimulators.

6.5a Mean latencies and interpeak amplitudes of VEPs from both eyes of subject IM's first recording using both stimulators

6.5b Mean latencies and interpeak amplitudes of VEPs from both eyes of subject IM' second recording using both stimulators.

6.6a Mean latencies and interpeak amplitudes of VEPs from both eyes of subject LO's first recording using both stimulators

6.6b Mean latencies and interpeak amplitudes of VEPs from both eyes of subject LO's second recording using both stimulators.

6.7a Mean latencies and interpeak amplitudes of VEPs from both eyes of subject MS's first recording using both stimulators

6.7b Mean latencies and interpeak amplitudes of VEPs from both eyes of subject MS's second recording using both stimulators.

6.8a Mean latencies and interpeak amplitudes of VEPs from both eyes of subject RD's first recording using both stimulators

6.8b Mean latencies and interpeak amplitudes of VEPs from both eyes of subject RD's second recording using both stimulators.

6.9 Comparison absolute latencies and interpeak amplitudes of thesis' results versus current LED-Box and TV monitor stimulators 
6.10a Interpeak latencies from the main components of subject CL's first VEP recording from both stimulators.

6.10b Interpeak latencies from the main components of subject CL's second VEP recording from both stimulators.

6.11 a Interpeak latencies from the main components of subject CQ's first VEP recording from both stimulators

$6.11 \mathrm{~b}$ Interpeak latencies from the main components of subject CQ's second VEP recording from both stimulators.

6.12a Interpeak latencies from the main components of subject IM's first VEP recording from both stimulators. 84

$6.12 \mathrm{~b}$ Interpeak latencies from the main components of subject IM's second VEP recording from both stimulators.

6.13a Interpeak latencies from the main components of subject LO's first VEP recording from both stimulators

6.13b Interpeak latencies from the main components of subject LO's second VEP recording from both stimulators.

6.14a Interpeak latencies from the main components of subject MS's first VEP recording from both stimulators

6.14b Interpeak latencies from the main components of subject MS's second VEP recording from both stimulators

6.15a Interpeak latencies from the main components of subject RD's first VEP recording from both stimulators

$6.15 \mathrm{~b}$ Interpeak latencies from the main components of subject RD's second VEP recording from both stimulators

6.16 Cross correlation results from VEP recordings 


\section{CHAPTER 1 - INTRODUCTION}

\subsection{Problem definition}

The main objective of this research is to design, and evaluate a new microprocessor-controlled VEP checkerboard pattern reversal Light Emitting Diode (LED) stimulator mounted on a goggle, and compare it with a current VEP checkerboard pattern LED-stimulator mounted on a utility box. The proposed stimulator would give clinicians the choice between two types of pattern reversal checkerboard LED-stimulators with different characteristics. After the design and implementation of the two stimulators their relative advantages were investigated. The major benefit of using the pattern-reversal checkerboard LED-goggle stimulator would be to obtain higher amplitudes on the major peaks of a visual evoked potential response. A quantitative performance evaluation is sought. The parameters used for the evaluation are absolute VEP latencies and interpeak VEP latencies and amplitudes, signal-to-noise ratio (SNR) and cross correlation between sequential recordings.

\subsection{Background}

Visual Evoked Potentials (VEPs), are scalp-recorded electrical responses of the brain elicited by visual stimuli. VEPs are obtained by recording the Electroencephalographic activity (EEG), using digital signal averaging techniques (synchronized time averaging). There are two types of VEPs, transient visual evoked and steady-state visual evoked potentials; transient VEPs are recorded using relatively 
low stimulation frequency rates ( 1 or 2 per second). On the other hand, steady state VEPs are recorded at high stimulation frequency rate (above 5 per second), these responses overlap one another and merge into quasi-sinusoidal oscillations that remain constant for the duration of the stimulation. Visual evoked potentials are also classified by its stimulus content into VEP responses to checkerboard patterns, diffuse light, and sine wave grating.

A VEP is a waveform composed of multiple peaks, which are generated by various structures along the visual pathway. The most significant peak occurs at a latency of 90-110 milli-seconds and an amplitude of about 10 micro-volts. This peak is often referred to as P1 or P100. This peak is preceded by a smaller negative peak at about 60-80 milli-seconds; it is referred to as N1 or N75, and followed by a second negative peak at about 140-145 milli-seconds, N2 or N145 (Fig. 1.1). P100, N75 and N145 are generated at cortical sites (Jeffreys, 1977). The earliest wave is P50, occurring at a latency of approximately 50 milli-seconds. This peak is 10 milli-seconds later than the earliest flash responses that can be recorded directly from the optic nerve. In 1972 , Jeffreys and Axford, used a dipole-like model to explain that N1 or N75 originates from the negative surface activities in the striate visual cortex; located mainly on the medial surfaces in and around the calcarine fissures. P1 or P100 is produced by the negative surface activities in the extrastriate visual cortex located mainly on the outer surfaces of the occipital lobes $\mathrm{N} 2$ or $\mathrm{N} 145$, is also originated in the extrastriate cortex but not in the same regions as P100 (Jeffreys, 1977). 


\section{Visual Evoked Potential}

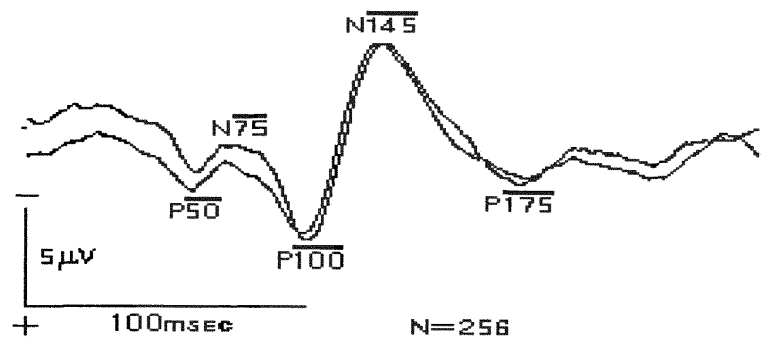

Figure 1.1. A normal pattern reversal checkerboard VEP, recorded in an $\mathrm{O}_{\mathrm{z}}-\mathrm{C}_{\mathrm{z}}$ derivation. LED stimulator with $40^{\prime}$ check size, $4^{\circ}$ by $4^{\circ}$ field size; filter setting $1.0-100$ $\mathrm{Hz} ; 256$ samples (adapted from Daly et al., 1990).

Flash or diffuse light VEPs are characterized by three components with different latencies. The primary component occurs at $29+/-2$ milli-seconds; the secondary component occurs at about 90 milli-seconds; the third component is a rhythmic after discharge that occurs from 135 to 350 milli-seconds and has no known clinical significance (Cyganek, 1961). Normal flash VEPs usually consist of seven peaks (Fig. 1.2) but the flash VEPs vary between subjects, and even in the same subject. These discrepancies are partly due to different stimulating and recording methods used in the testing laboratories. For clinical diagnostic purposes, only two components of the flash VEPs are used clinically. These are the latency of the major positive peak that occurs 
between 50 and 100 milli-seconds after the flash; and the latency of the following negative peak, that occurs between 100 and 250 milli-seconds (Spehlmann, 1985).

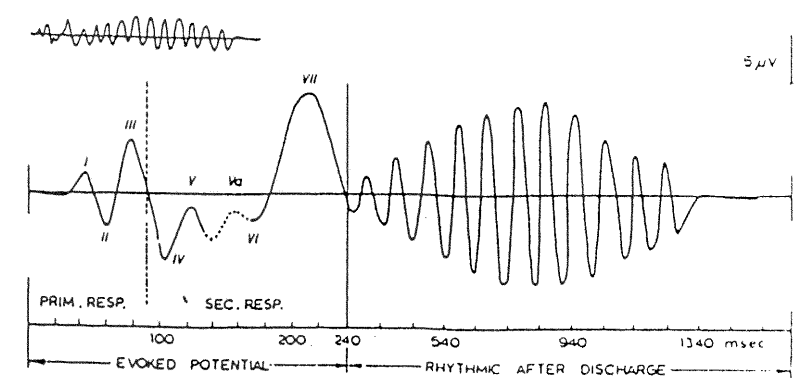

Figure 1.2. A normal flash or diffuse light VEP averaged from 75 subjects. The VEP response is divided into a primary response, a secondary response and is followed by a rhythmical after discharge. Note the change in time scale between VEP and the after discharge. The VEP and the after discharge shown in the upper left corner are on the same time scale. Recording between Midoccipital and Midpariental electrodes. Negativity at the occipital electrode is plotted upward. (from Ciganek, 1961).

Sine wave grating VEPs are characterized by the change of luminance in the display device according to a sine wave. These stimuli consist of alternating light and dark stripes with midlines of maximum and minimum luminance and gradual luminance between them. With such stimuli the effects of luminance change and the mean luminance level can be studied separately. 
There are other types of stimuli such as: bar grating, small macular light spots, moving and stereoscopic random dot patterns. Bar grating consists of light and dark stripes of equal width with sharp borders between them; like pattern checkerboard VEPs, bar grating VEPs also depend on the size of the stimulus element, the contrast between light and dark elements, and the stimulus rate. However, bar grating VEPs have been rarely used in clinical studies; they are used in ophthalmology for refraction, biocularity and astigmatism studies (Spehlmann, 1985).

In 1993 Celesia, et al. recommended standards for electroretinograms and visual evoked potentials to improve clinical care and to permit comparison among different laboratories. Most of the guidelines represent the minimal requirements for recording basic visual evoked responses in any laboratory. The recordings made followed such standards. (See Chapter 5 - Experimental Methods).

\subsection{Preface to chapters}

This thesis is organized in the following manner: In Chapter 2 (Electrophysiology and VEP Characteristics), a brief description of the anatomy and physiology of the human eye is presented. A condensed explanation of the generation of pattern reversal checkerboard visual evoked potentials, their classifications and their clinical applications are also presented. In Chapter 3 (Pattern Checkerboard Reversal VEP Stimulators), a review of current pattern reversal stimulators is included. The goal and objective of this research are presented in Chapter 4 (Goals and Objectives). The hardware 
instrumentation, data acquisition parameters, patient testing techniques, response labeling and analysis are discussed in Chapter 5 (Experimental Methods). In Chapter 6 (Results), the obtained VEPs, their absolute latencies, interpeak latencies, and signal-to-noise response plots are presented. The comparison between the two proposed stimulators is covered in Chapter 7 (Discussion). Chapter 8 (Conclusion) summarizes the accomplishments of the study and possible future developments and improvements. 


\section{CHAPTER 2 - ELECTROPHYSIOLOGY AND VEP CHARACTERISTICS}

The structure of the human eye is shown in Fig. 2.1. The outer coat of the eye is known as the sclera. The cornea is the transparent region of the sclera. Inside the sclera is found a vascular layer, known as the choroid, which is usually pigmented. The lens are found in the interior of the eye; the aqueous humour is found in front of the lens; and the vitreous humor behind it. In front of the lens is the diaphragm, the iris, and the pupil whose aperture diameter can be varied by contraction of the iris muscle. Thus the activity of the iris can regulate the amount of light entering the eye (Aidley, 1989).

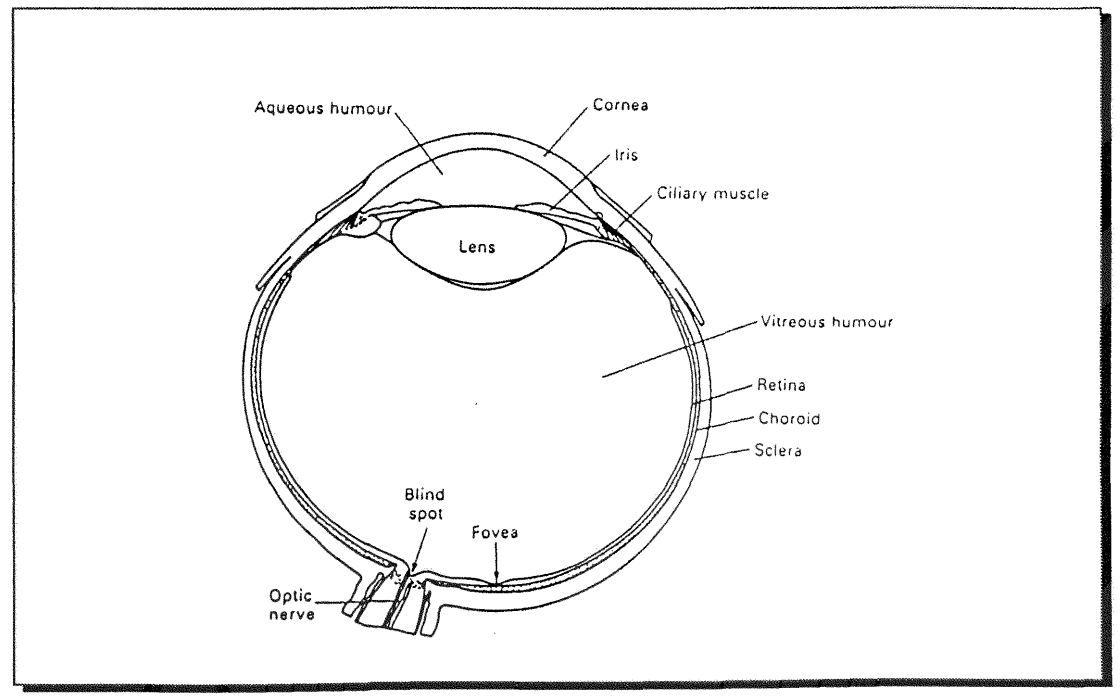

Figure 2.1. A horizontal section of the human eye (Aidley, 1989). 
The human retina is composed of photoreceptor cells known as rods and cones. Rods, found in the peripheral retina, are more sensitive than cones concerning dim light. In dim light, where rod output dominates, the physiological state of the retina is referred to as scotopic. Cones, concentrated in the macular region of the eye, detect color and adapt to brighter light. In bright light, where cone output dominates, the physiological state is referred to as photopic. Cones sensitive to red light are found in the macular region, whereas cones sensitive to blue light are encountered outside this region. The outputs of the cones and rods interact with bipolar and ganglion cells whose axons constitute the retina's nerve fiber layer and the optic nerve (Epstein, 1990). See Fig. 2.2. 


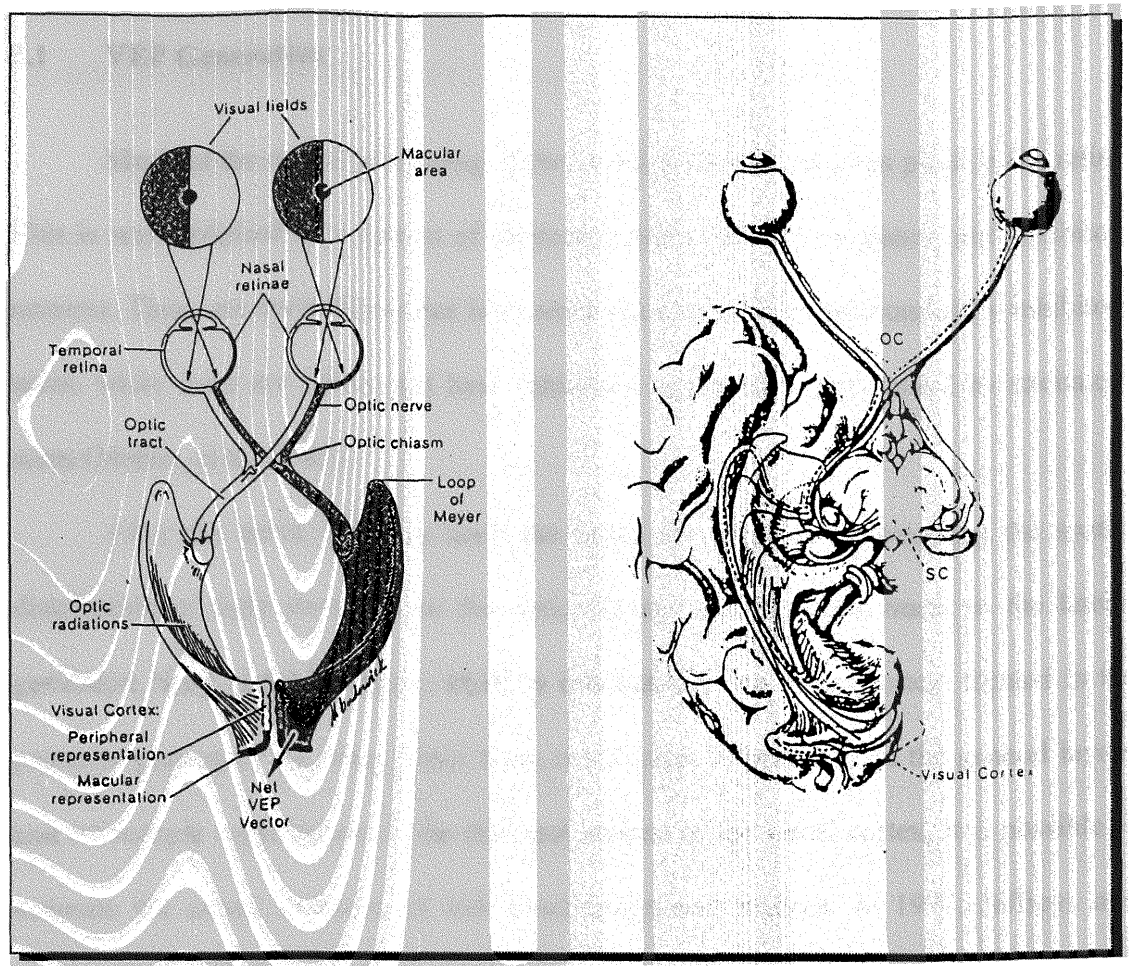

Figure 2.2 The primary visual pathways, left hand side picture illustrates the crossing of temporal fibers in the optic chiasm, the macular and peripheral retinal projections to the striate cortex, and the direction of the net VEP vector from hemifield stimulation obliquely back across the opposite hemisphere (from Epstein, 1990). Right hand-side picture illustrates the optic chiasm (OC), the superior colliculus (SC), and the lateral ventricle (LV) (from Moller et al, 1985). 


\subsection{VEP Generation}

Much of the neural processing of the visual field systems takes place in the retina. This is accomplished by a pattern of interconnections between excitatory and inhibitory neurons. There are nerve fibers that have small excitatory fields surrounded by inhibitory areas, while there are others that have inhibitory center areas surrounded by excitatory areas (Moller, et al., 1985).

After the nerve impulses leave the retina the impulses representing the spatial distribution of light travels from the optic chiasm into the optic tracts to the lateral geniculate body in the thalamus, which is connected to the visual cortex, located in the posterior portion of the brain. Since there is sufficient information on the general layout and retinotopic organization of the different regions of the visual cortex, it is possible to estimate the actual locations of individual component sources. In 1972, Jeffreys and Axford, used a dipole-like model to explain that N1 or N75 originates from the negative surface activities in the striate visual cortex; located mainly on the medial surfaces in and around the calcarine fissures. $\mathrm{P} 1$ or $\mathrm{P} 100$ is produced by the negative surface activities in the extrastriate visual cortex located mainly on the outer surfaces of the occipital lobes. $\mathrm{N} 2$ or N145, is also originated in the extrastriate cortex but not in the same regions as P100 (Jeffreys, 1977). But it has not yet been possible to estimate the actual sites of origins of P100 and N145 with the extrastriate cortical regions, because of insufficient 
information on the distributions of these components on the layout and organization of the different extrastriate regions of the human visual cortex. See Fig. 2.3.

There are two types of cortical processes that contribute to these three components of the VEP: (a) contrast-specific mechanisms, which contribute solely to N75, and (b) contour-specific mechanisms, which provide the predominant contributions to P100 and N145, but also contribute to N75. Contrast-specific processes are sensitive to the pattern stimuli but not to overall luminance changes. These mechanisms are relatively unadaptive and seem to be mainly biocularly sensitive; they appear to be confined to the striate cortex.

Contour-specific processes react better to pattern stimuli with well-defined contours and are less sensitive to the actual contrast level. These mechanisms respond only to the onset of the stimulus and are highly adaptive. They are both orientationspecific and dimension-specific being sensitive to discontinuous contours and predominate in the extrastriate regions but are also present in the striate cortex. Using these component-analysis techniques show that components of the VEPs to brief pattern presentations appear to be the most appropriate method for studying cortical processes (Spekreijse, et al. 1977). 


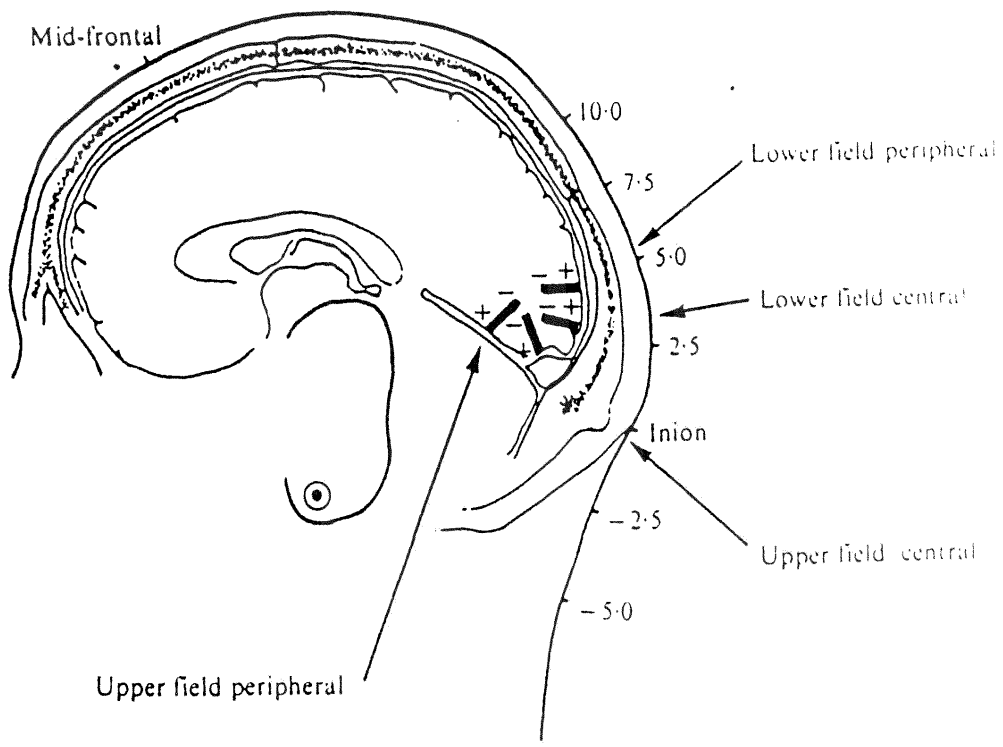

Figure 2.3 Diagrammatic representation of the relative positions and orientations of the hypothetical dipoles representing the central and peripheral parts of the upper and lower visual field. The central and peripheral upper fields are oriented at right-angle to each other. These suggested orientations would explain the surface positivity recorded at lower occipital electrodes in response to the central upper-filed stimulus when using mid-frontal reference (from Halliday et al., 1977). 


\subsection{Acquisition of VEPs}

A single VEP response to a pattern stimulus has a low amplitude and usually is partially or completely hidden by ongoing spontaneous Electroencephalographic (EEG) activity. Each response is elicited repeatedly and averaged with an automatic data acquisition system, such as a Digital Signal Processor (DSP). This method extracts the response component that is time-locked to the stimulus and eliminates the EEG component that is not synchronized to the stimulus. This averaging technique achieves an increase in signal-to-noise ratio by sequentially adding repetitive signals.

Although other stimulation patterns, such as diffuse, sinusoidal grating, etc. are also used to elicit VEPs, the focus of this research will be the checkerboard patterns that are most commonly used. A checkerboard pattern consists of light and dark squares with sharp borders. Sine wave grating, on the other hand, consists of light and dark stripes with a gradual transition of brightness between them. Pattern VEPs are due mainly to the visual content of the pattern, specially the density of the light and dark contrast borders. Diffuse or flash VEPs are due to changes of luminance only.

The stimulus rate can be categorized into two types: transient and steady-state VEPs. Transient VEPs involve a sequence of different peaks that occur at a constant latency after each stimulus. Transient VEPs are recorded using relatively low stimulation frequency rates ( 1 or 2 per seconds). On the other hand, steady state VEPs involve a rhythm of uniform peaks occurring at the same frequency or at the same harmonic frequencies; they are recorded at high stimulation frequency rate, these responses overlap 
one another and merge into quasi-sinusoidal oscillations that remain constant for the duration of the stimulation.

The presentation mode can be classified as pattern reversal, pattern appearance and disappearance, and diffused lights. In pattern reversal, the stimulus consists of a sudden change of all light pattern components into dark ones, or vice versa. Pattern appearance and disappearance are produced by presenting a pattern alternating with a diffusely illuminated or dark field, either the onset or the offset is used as a stimulus. (Spehlman, 1975).

Transient VEPs are preferred over steady-state VEPs in view that their responses are more defined. Furthermore, pattern VEPs have less variable between subjects compared to diffuse VEPs and are more sensitive to lesions that impair conduction through the visual pathway. In addition, pattern VEPs have been used for investigating chiasmal and retrochiasmal lesions, as well as, ophthalmological testing of visual acuity and refractory errors. For these reasons pattern VEPs are generally preferred to flash or diffuse VEPs. 


\subsection{Clinical Applications}

Recording of VEP is extensively used in patients with disorders of the sensory visual pathway and on patients at risk of visual pathway damage. The use of VEPs is advantageous for young children and infants who cannot communicate visual symptoms or cooperate during standard vision assessment. Furthermore, the VEP recordings can be used for detecting lesions caused by dysfunction of the sensory pathways from retrobulbar neuritis or optic atrophy; likewise, for confirming functional loss when disorders are present in the visual system. Furthermore, VEP recordings can be also very useful in the prognosis of multiple sclerosis (MS) from the late interpeak latency differences obtained from the MS subject's recordings in comparison with normal subjects. In addition, VEP recordings are also useful in finding tumors compressing the optic nerve, ischemic neuropathy of the optic nerve, and some ophthalmological disorders. 


\section{CHAPTER 3 - CHECKERBOARD PATTERN REVERSAL VEP STIMULATORS}

Checkerboard pattern reversal is now recognized as the stimulus of choice for eliciting VEPs. A variety of devices for generating pattern reversal exist; they range from television monitors to arrays of LEDs (Pratt, et al.; 1984).

\subsection{Television Screen Pattern Stimulator}

There are several TV pattern stimulators that elicit VEP responses. These TV pattern stimulators have several advantages such as: they are sufficiently large and bright, they can generate checkerboard patterns, as well as, horizontal and vertical bars of different sizes and present them at different rates, they may be used to visually stimulate half fields or quadrants without shift of a fixation point. The stimulus pattern may be mixed with a TV program, such as cartoons, to keep young children focusing at the screen without significant degradation of the VEP (Sokol, 1977).

On the other hand, TV pattern generators have some disadvantages such as: contrast borders between dark and light are not sharp, the control of contrast and luminance is limited and not linear. They are also limited in reversal time and accessibility to the patient's bed-side.

\subsection{Slide-projector Stimulator}

An ordinary Kodak carrousel, $5 \times 5 \mathrm{~cm}$, slide projector has been used as a pattern stimulator. The image is presented to the screen through a high-speed mirror 
galvanometer, placed near the projector, and controlled by an electro-mechanical transducer. By turning the mirror through a small angle, the pattern moves from side to side across the screen (Desmedt, 1977). This rapid displacement of a checkerboard or vertical grating through one half-cycle produces a reversal of the pattern. Some of the advantages of this projector method is the ease and convenience with which slides can be made and changed to vary the pattern, the relative low cost, the flexibility of viewing distance and field size. The disadvantages of this method are the difficulty of controlling contrast, and the limited luminance of the projector lamp or deterioration of the projector lamp over the years.

\subsection{Rotating Polaroid stimulator}

A $30 \times 30$ square array can be constructed out of strips of Polaroid. The squares are assembled between two plates of glass bound with tape. The pattern reversal occurs when the square array is viewed through a second Polaroid disc spun before the eye. The advantages of this method are that the system is portable and robust, no fixation point is needed, interpretation of the response depends on amplitude and phase information only, and a precise pattern reversal can be obtained. On the other hand this method produces only sinusoidal changes in contrast, and the pattern stimulation is done mechanically. 


\subsection{Patterned mirror stimulator}

The pattern stimulus is obtained by the reflecting and transparent elements of a pattern mirror. The luminance of the two sets of elements is provided by separate light sources, two fluorescent tubes, with brightness levels that can be modulated independently. The advantages of this method are that a large range of luminance levels can be produced, the temporal modulation waveform can be controlled, and that luminance modulation and spectral compositions are independently controlled for each of the sets of spatial elements. The disadvantages of this technique are that the pattern is set at an angle of $45^{\circ}$ which limits the visual angle. In addition, the spatial parameters cannot be changed during stimulation without adding other optical equipment, and there are color differences between the transmitted and reflected light.

\subsection{Tachistoscopic display stimulator}

This stimulator consists of four perplex fields- each one back-illuminated by three blue fluorescent tubes and optically superimposed by half-silvered mirrors. Each field is independently switched by externally controlled bistable units. The stimulus is the alternation of the test field and the reference field.

The advantages of this technique are that pattern onset-offset, the pattern reversal, and the pattern displacement are possible at constant luminance; different forms of pattern can be presented in any region of the field and at the same run; accurate control of duration and high contrast level can be achieved. On the other hand, the disadvantages of 
this method are that the spatial configuration of the pattern can not be externally controlled. In addition; pattern alignment presents some difficulty and background illumination changes on switching. Furthermore, each pattern has to be drawn or prepared photographically and the usage of blue fluorescent tubes makes it unsuitable for color experiments.

\subsection{Maxwellian-view stimulator}

The stimulus is viewed through an eyepiece that focuses the light from a pattern so that it forms an image at the level of the pupil and becomes independent of pupillary size (Spelhmann, 1984). The advantages of this method are that the stimulus parameters such as luminance, field size, duration, etc., are more efficiently controlled on the retina of the subject. Among the disadvantages of the Maxwellian-view stimulators are the fact that they are relatively complicated to construct and require that the subject's eye be accurately aligned with their optics.

\subsection{Fiber Optic Display stimulator}

A pattern stimulator using fiber optic consists typically of an $8 \times 8$ matrix of LEDs. An assembly of optical fibers can be arranged in a desired pattern (Pratt, et al., 1984). This stimulator may use different color patterns, and it is small and inexpensive. On the other hand, the size and arrangement of the pattern element are fixed, and the size of the elements can only be altered by changing the viewing distance. 


\subsection{Light Emitting Diode's Matrix Array stimulator}

In 1974 Evans introduced LEDs as stimuli; this method was later used by several researchers (Nilsson 1978; Epstein 1979). LEDs can be mounted in $6 \times 6,8 \times 8,12 \times 12$, or $21 \times 21$ square matrices. The LED matrix can be enclosed in a small utility box mounted on a mechanical arm, or a microphone stand. Some of the previously pattern reversal stimulators have limitations regarding: pattern, field size, reversal time, color, durability, versatility, and luminance. The LED arrays overcome some of these limitations such as: color, durability, and versatility. (Epstein, 1979). The disadvantages of this method are the LED size, low luminance, and contrast borders that are often not sharp; other drawbacks are the large number of LEDs necessary to use in the stimulator, and the high power required to drive the stimulator. On the other hand, they elicit evoked responses comparable in amplitude and waveform to those obtainable by a TV pattern stimulator (Epstein, 1979).

Comparison of VEPs elicited by LEDs and TV monitors has been performed by Epstein (1979), and Andersson and Siden (1994) in patients with multiple sclerosis. Andersson and Siden found that more abnormalities were detected with red LED pattern stimulation. The latencies to all components were found to be longer with LED stimulation, probably due to lower luminance of the LED pattern. Andersson and Siden also concluded that LED stimulation elicits P100 potentials with longer latencies but equal amplitude compared to TV stimulation. Andersson and Siden concluded that LED stimuli were more sensitive in detecting visual system dysfunction and that the most 
discriminating factors between stimuli were luminance and color. Both LED and TV monitor stimulator are currently being used clinically. 


\section{CHAPTER 4 - GOALS AND OBJECTIVES}

The goal of this research is to design, evaluate and compare two Visual Evoked Potential checkerboard pattern stimulators (a 12 x 12 LED array mounted on a utility box, and two $8 \times 8$ LED arrays mounted on the eyepiece of a goggle). The first step required to achieve this objective was to design and build both stimulators and the second, to test both stimulators on subjects. Under most clinical settings, two recordings are used to confirm the presence or absence of any particular peak. The performance is to be tested using recordings from subjects with normal VEPs. Furthermore, the VEP responses are compared by generating peak latencies, interpeak latencies, signal-to-noise ratio (SNR) estimations and cross correlation tables from sequential recordings.

Ultimately, the quantitative measures of performance obtained from both stimulators will be used to point out their relative advantages and disadvantages. The results of this work will provide an objective indication of the applicability of the stimulators for potential clinical use. 


\section{CHAPTER 5 - EXPERIMENTAL METHODS}

\subsection{Hardware instrumentation}

The hardware of the Visual Evoked Potential acquisition system, shown in Figure 5.1, was composed of a two-channel bioamplifier, a programmable Opti-Amp Receiver Board, a Digital Signal Processor (DSP) TMS320C31 Board, an IBM Compatible Personal Computer (PC), and the two VEP Stimulators designed for this work. The stimulators were designed to be controlled by the DSP processor. The bioamplifier used was a battery-powered IHS TXDF amplifier. This amplifier had a differential input requiring 3 electrodes: positive, negative and ground. The amplifier had a pre-amplifier amplification of 2000, a 10 megaohm input impedance, and was optically connected to the Receiver board with a 25 foot fiber optic cable. The Opti-Amp Receiver board provided a programmable High-Pass Filter $(1-300 \mathrm{~Hz})$ with $-6 \mathrm{~dB} /$ octave roll-off slope, a programmable Low-Pass Filter $(100-5000 \mathrm{~Hz})$ with $-6 \mathrm{~dB}$ /octave roll-off slope, a programmable Gain Stage (250 - 1500 times) for the total gain of 50,000 - 300,000 including the initial gain from the bioamplifier, and an Anti-aliasing Filter Section $(10,000 \mathrm{~Hz})$ with $-12 \mathrm{~dB} /$ octave roll-off slope. The measured noise level was 1 microvolt

peak to peak when all inputs were shorted (1 - 3000 Hz bandwidth). A 14-line ribbon cable connects the Opti-Amp Receiver board to the DSP board.

The DSP board was responsible for stimulus presentation and data acquisition. The DSP board is interfaced to the stimulators through a programmable Peripheral 
Input/output (PIO) device. The checkerboard patterns were stored in each VEP stimulator, and were digitally selected by software running on an IBM computer and transferred to the DSP's PIO for presentation. The board contains a DB25 connector that is used to connect the VEP stimulators. The DSP board also provided up to 4 input channels. Each channel was supplied with an Analog to Digital (A/D) converter with a maximum peak to peak input voltage of 10 volts. The sampling rate was programmed for an $800 \mu \mathrm{sec}$ sampling period. A fixed sampling window of 512 pre- and 512 poststimulus points was used. Furthermore, the DSP board was supplied with $4 \mathrm{~K}$ of dual port memory that allowed transfer of data to the PC. This particular feature allowed the PC to multitask with different functions such as: updating the sweeps, displaying the VEP being averaged, checking on the artifacts counts, allowing the user to label each peak, etc., while the DSP board was averaging and issuing the stimuli. Fig. 5.2 shows the connections between the DSP and Receiver boards. 


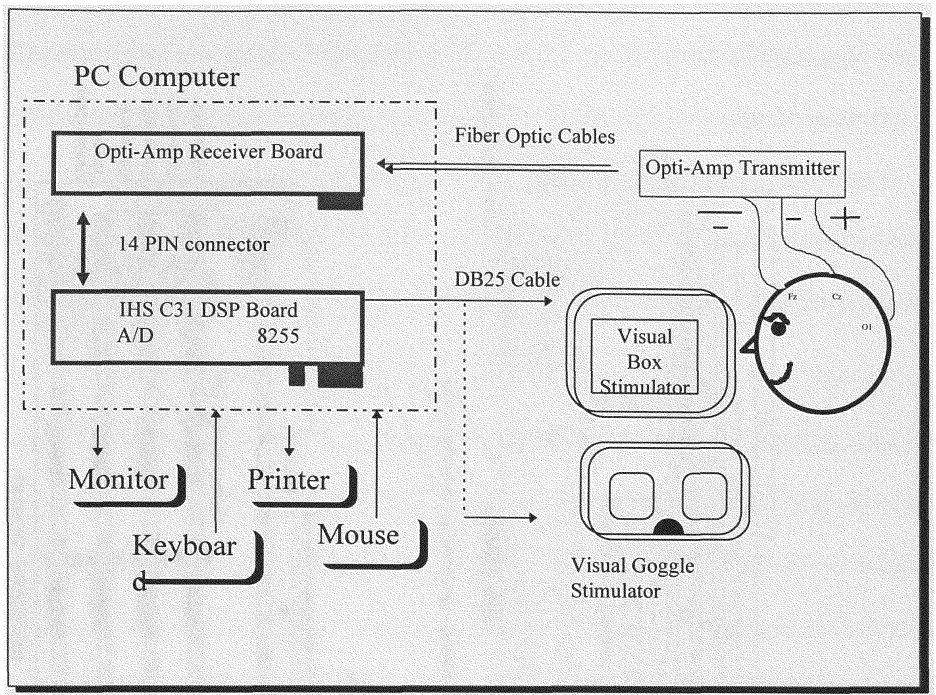

Figure 5.1 Schematic diagram of the visual evoked potential system used to acquire recordings from normal subjects. 
RECEIVER BOARD

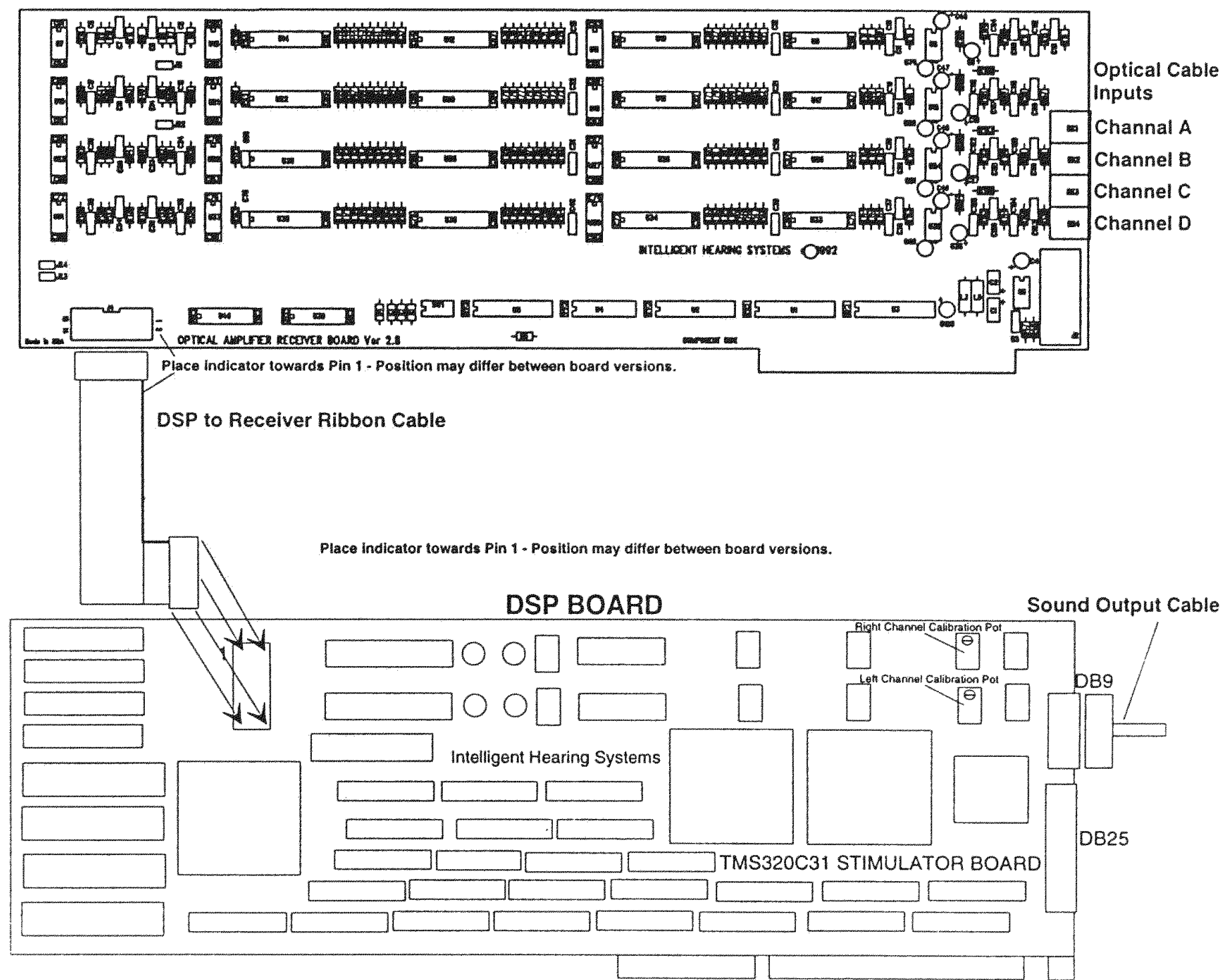


The software running on the DSP board was developed in C (TMS-320C30 C Compiler Reference Guide, Texas Instruments, 1990). The Smart-EP VEP program was subdivided into a series of subroutines that were called through the hardware interrupts (See Fig. 5.3). The software running on the PC was developed in Pascal (Turbo Pascal ver. 7.0, Borland International, 1992). The PC software acted as an intermediary between the user and the DSP board. This dual architecture provided a versatile hardware system.

Smart-EP VEP is a program consisting of multiple function calls such as: set artifact level, set VEP pattern, set high/low pass filters, set gain, set sampling rate, set inter stimulus interval (ISI), return average, etc. Fig. 5.3 shows the block diagram of the data acquisition, and the timing diagram and data algorithm of the averaging technique. Each function call performs a specific task. However, the "set ISI" function performs the interrupt routine which gathers and stores the single sweep data into the DPS's memory. The timing diagram shows the time used to gather the pre- and post- stimulus data as well as the time for the ISI function. Figure $5.3 \mathrm{~b}$ shows the flow chart of the ISI function. 


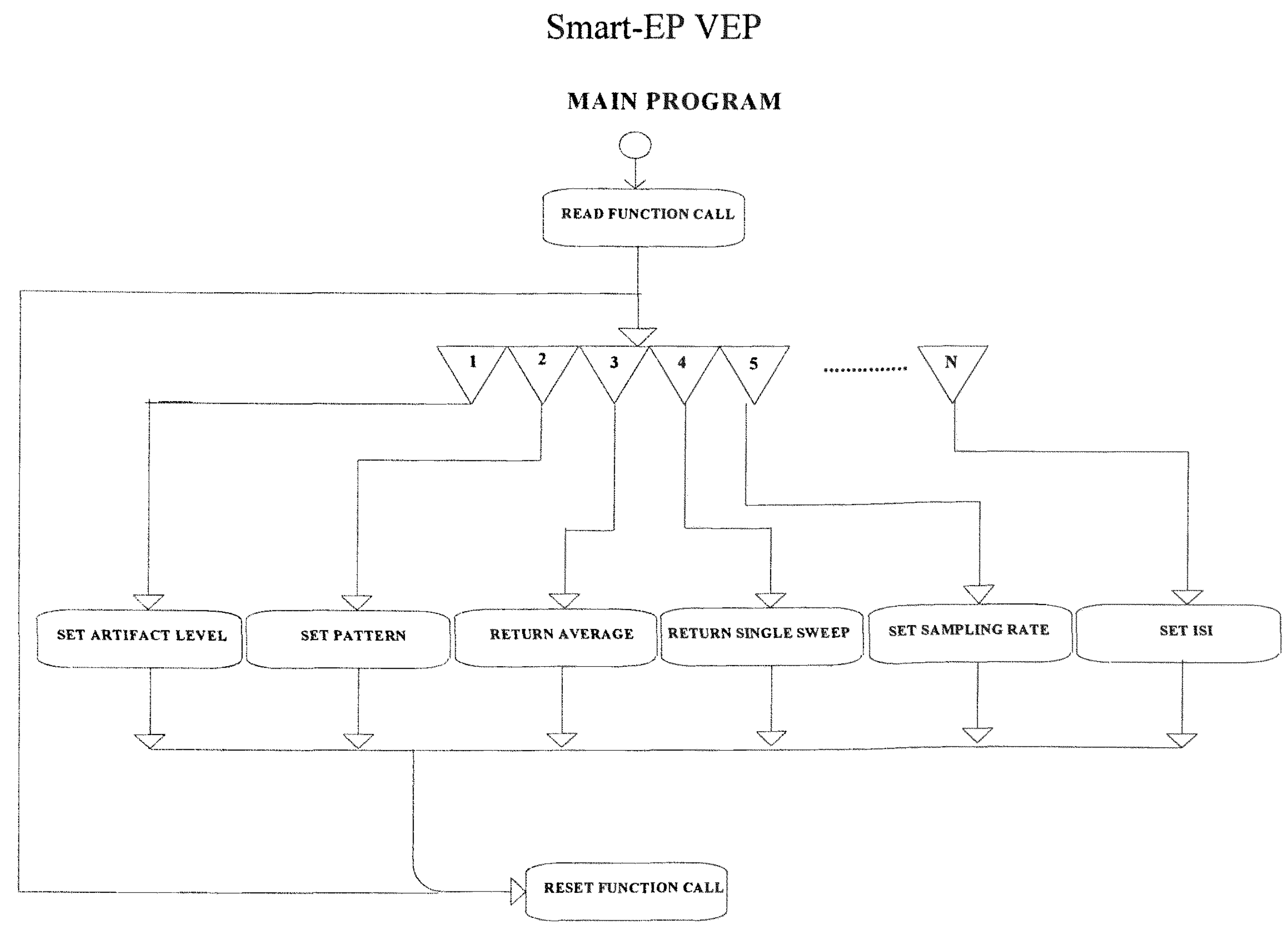

DATA DICTIONARY

ISI INTER STIMULUS INTERVAL

SS SINGLE SWEEP

TIMING DIAGRAM

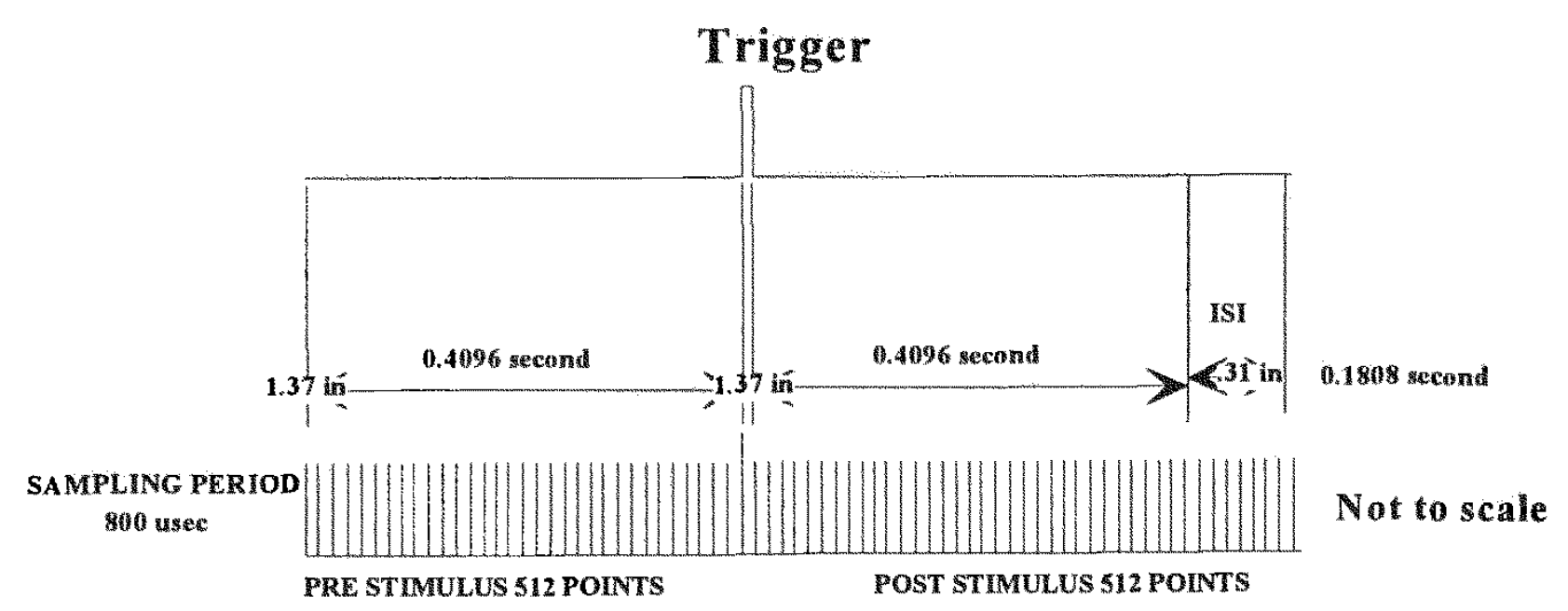

Figure 5.3a Block diagram of the data acquisition and timing for the Smart EP VEP. 


\section{INTERRUPT ROUTINE}

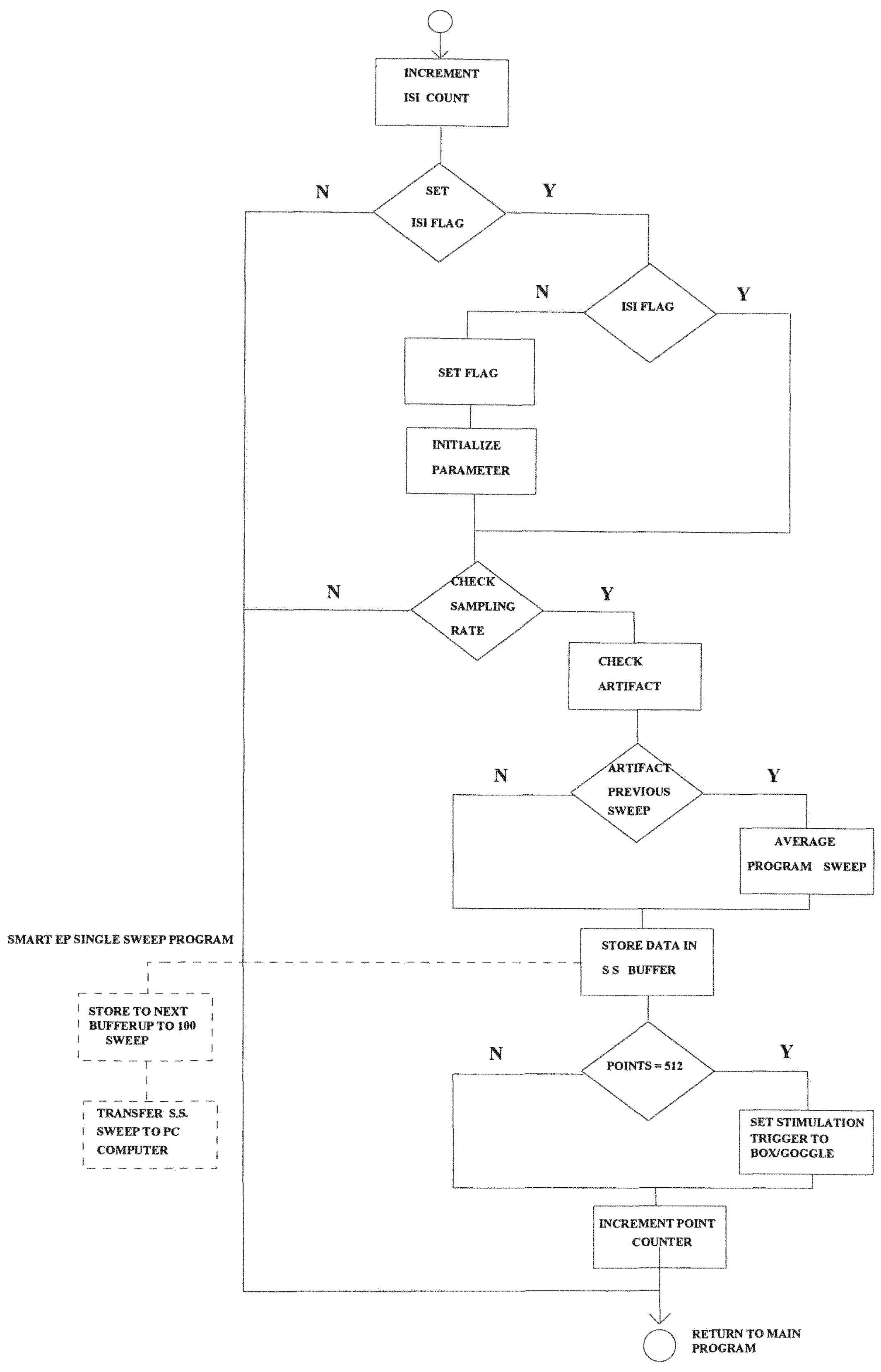

Figure 5.3b Flow chart of the Set Inter Stimulus Interval Function. 
The checkerboard pattern-reversal Visual Box stimulator allowed the user to program up to 256 patterns and save them in twelve reprogrammable CMOS EPROMs $(65,536 \times 16 \mathrm{Bit})$. The Visual Box stimulator contains 144 high-efficiency, $5 \mathrm{~mm}$ square, red LEDs; and a low power, $3 \mathrm{~mm}$ round, green LED in the center of the array. This center point LED is used as a fixation point for the user to look at when the test is being performed. The current through each LED is controlled by a $220 \Omega$ resistor. Fig. 5.4 shows the block diagram of this stimulator. This circuit is enclosed in a $220 \mathrm{~mm} \times 35 \mathrm{~mm}$ x $230 \mathrm{~mm}$ gray box. A DB25 male-to-female cable connects the VEP stimulator to the DSP board as is shown in Fig. 5.4. 
From DSP

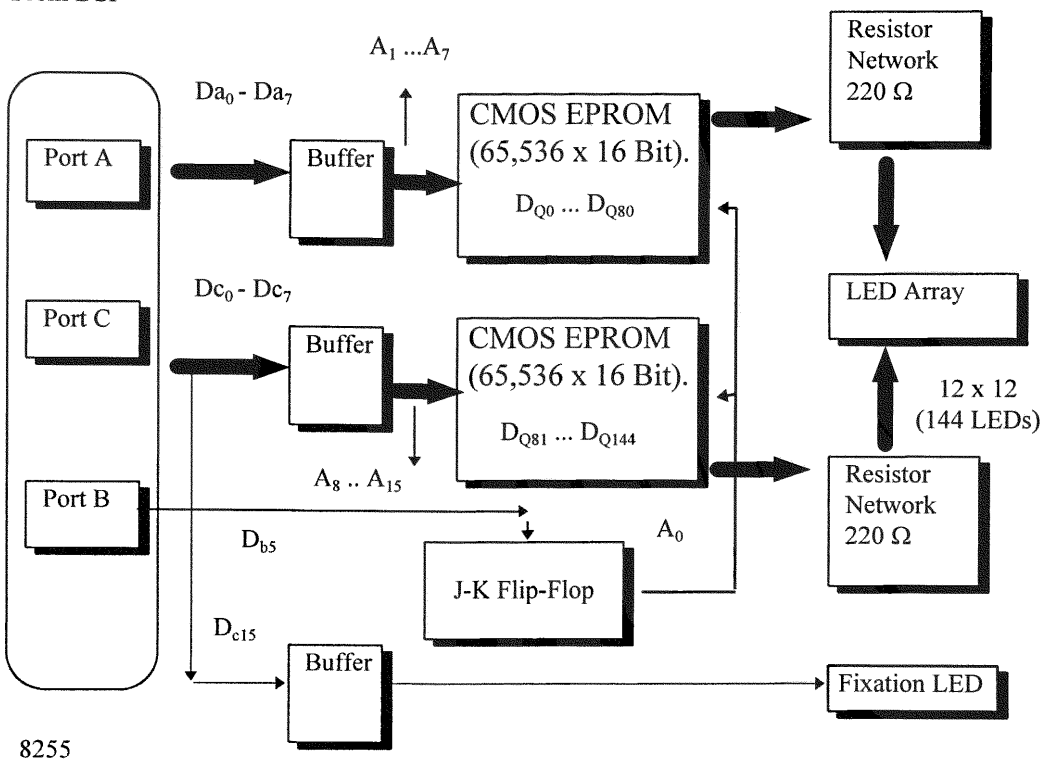

DB25

Figure 5.4 A block diagram of the Visual Box Stimulator.

The Intel $82 \mathrm{C} 55 \mathrm{~A}$ is a high performance, general purpose programmable $\mathrm{I} / \mathrm{O}$ device that is designed for use with most microprocessors- including the TMSC $320 \mathrm{C} 31$ DSP processor. The $82 \mathrm{C} 55 \mathrm{~A}$ provides $24 \mathrm{I} / \mathrm{O}$ pins which be individually programmed in two groups of 12 and used in 3 modes of operations. The three basic modes of operation that can be selected are: 
Mode 0 - Basic Input/Output

Mode 1 - Strobed Input/Ouput

Mode 2- Bi-directional Bus

The Mode 0 (Basic Input/Output) provides simple input or output operations for each of the three ports. No handshaking is required, data are simply written to or read from a specified port. Ports A, B, C were programmed to Mode 0 , output configuration, by the TMS C 31 processor.

In Mode 0, Port A and B transfer data to the TMS27C210 EPROMs. Port C generates the trigger signal to a J-K edge-triggering flip-flop; this versatile flip-flop toggles the address $A_{0}$ in each EPROM, therefore, the checkerboard pattern changes from right to left. In addition, port $\mathrm{C}$ controls the fixation point to be set on or off. The outputs on Port A, B, Cs deliver only $2.5 \mathrm{~mA}$, which is not sufficient to drive nine of the TMS27C210 EPROMs. Two octal buffers, 74F541, are needed as interface with the EPROMs. The EPROMs' output capability can drive all the 144 square LEDs.

The checkerboard pattern-reversal LED goggle stimulator also allows the user to program up to 512 patterns, 256 patterns for each eye, and these patterns are saved in eight one-time programmable CMOS PROMs (65,536 x 16 Bit). Each goggle eyepiece's array consists of 64 high-efficiency, $2 \mathrm{~mm}$ square, red LEDs with a $1.5 \mathrm{~mm}$ round green LED in the center of the array acting as the fixation point. The current also is controlled 
by a $220 \Omega$ resistor for each LED. The circuit is enclosed in a prototype goggle. The block diagram of this stimulator is shown in Figure 5.5.

The $82 \mathrm{C} 55 \mathrm{~A}$ interface for the LED-goggle stimulator is very similar to the one used for the LED-box. Port A and B transfer the data to the TMS27PC210 PROMs. Port $\mathrm{C}$ also generates the trigger signal, but controls two fixation points - right and left center points instead of one center point.

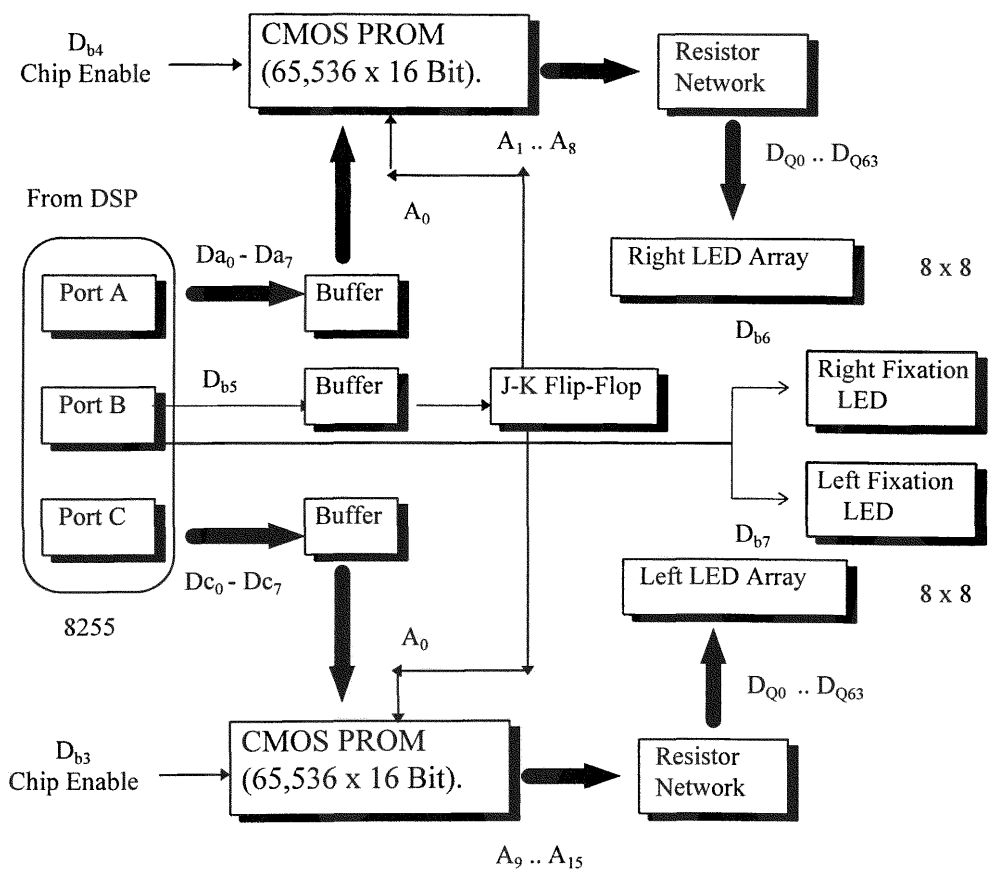

Figure 5.5 A block diagram of the Visual Goggle Stimulator 


\subsection{Design Constraints}

The main constraint of both designs is that either device can only be used with the Smart-EP DSP C31 board. A second constraint is that the brightness of each LED is fixed. However, it could be potentially changed by controlling the current through the resistor networks.

\subsection{Earlier design approaches}

Two earlier designs were constructed and tested. The first design consisted of one flip-flop connected to the base of sixty-four NPN transistors, but the design required an external power supply for driving the LEDs. This approach only generates one pattern configuration. The second approach consisted of four MM5486 LED display drivers; a single pin controls the LED display brightness by setting a reference current through a variable resistor connected to the power supply. The data bits were latched by the positive level of the trigger, thus providing non-multiplexed, direct drive to the LED array. Serial data transfer was needed to control the MM5486 LED display driver. Although the TMSC320C31 has a serial port; it was not accessible from the Smart-EP C31 DSP board. The first design was simple, but power consumption was the major constraint. In the second approach, power was not a factor, but the connectivity with the Smart-EP DSP was the major constraint. 


\subsection{Pattern Generation Algorithm}

A Pascal program was written for developing each VEP stimulator's patterns; from different size checkerboard patterns to vertical bar patterns. Each stimulator was programmed with full-field, half-field, and quarter-field patterns. Figure 5.6 shows the screen display of the VEP pattern generation program and the data flow diagram of the pattern generation. When a pattern set is built with this program, it can be factoryprogrammed into and PROM or EPROM and sent to the user.

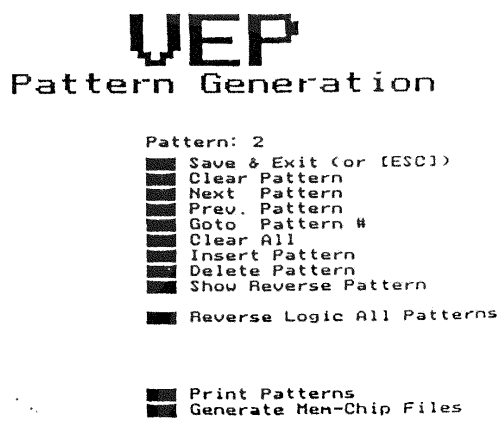

Figure 5.6a Screen display of the VEP's pattern generation program. 


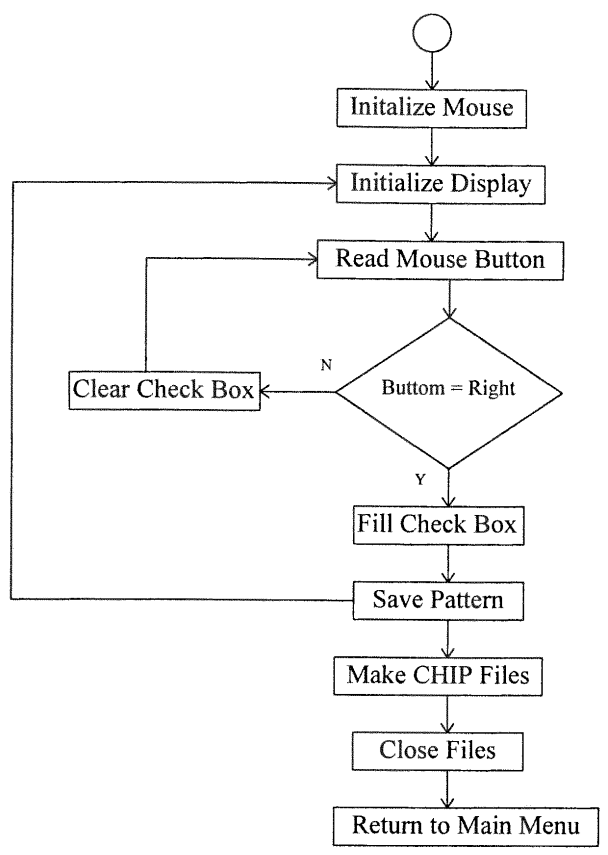

Figure 5.6b Flow diagram of the pattern generation program.

This program generates the binary file needed to store in each TMS27PC210 or TMS27C210 memory. An EPROM programmer, Hi-Low ALL-03A PC-based universal programmer and tester, was used to program the TMS27C210 and TMS27PC210.

\subsection{Data Acquisition Parameters}

All the recordings used in this thesis were obtained using the recommended standard clinical settings (Celesia et al. , 1993). Filter settings were maintained at $1 \mathrm{~Hz}$ for high pass and $300 \mathrm{~Hz}$ for low pass with $-6 \mathrm{~dB}$ per octave roll-off slopes. The amplifier 
was optically isolated from the patient. The analog signal was digitized with 1250 samples/sec per channel and a 16-bit resolution per sample.

\subsection{Patient testing}

Subjects of different ages and sight were used to obtain sample VEP responses. Four gold-cup EEG electrodes were placed, two recording electrodes were placed in the occiput area $\left(\mathrm{O}_{1}\right.$ and $\left.\mathrm{O}_{2}\right)$, a ground electrode on the forehead $\left(\mathrm{F}_{\mathrm{pz}}\right)$, and a reference electrode on the vertex $\left(\mathrm{C}_{\mathrm{z}}\right)$. The suggested montage assures the recording of a reproducible VEP, even in the occasional cases when the potential gradient of the visual response is very prominent at the vertex. At least two averages were obtained and superimposed to verify reproducibility of the results. Figure 5.7 shows the main EEG 1020 system electrodes, viewed from left (top left), right (bottom left), front (top right), and back (bottom right). The positions involved in the montage used for this work have been marked. 


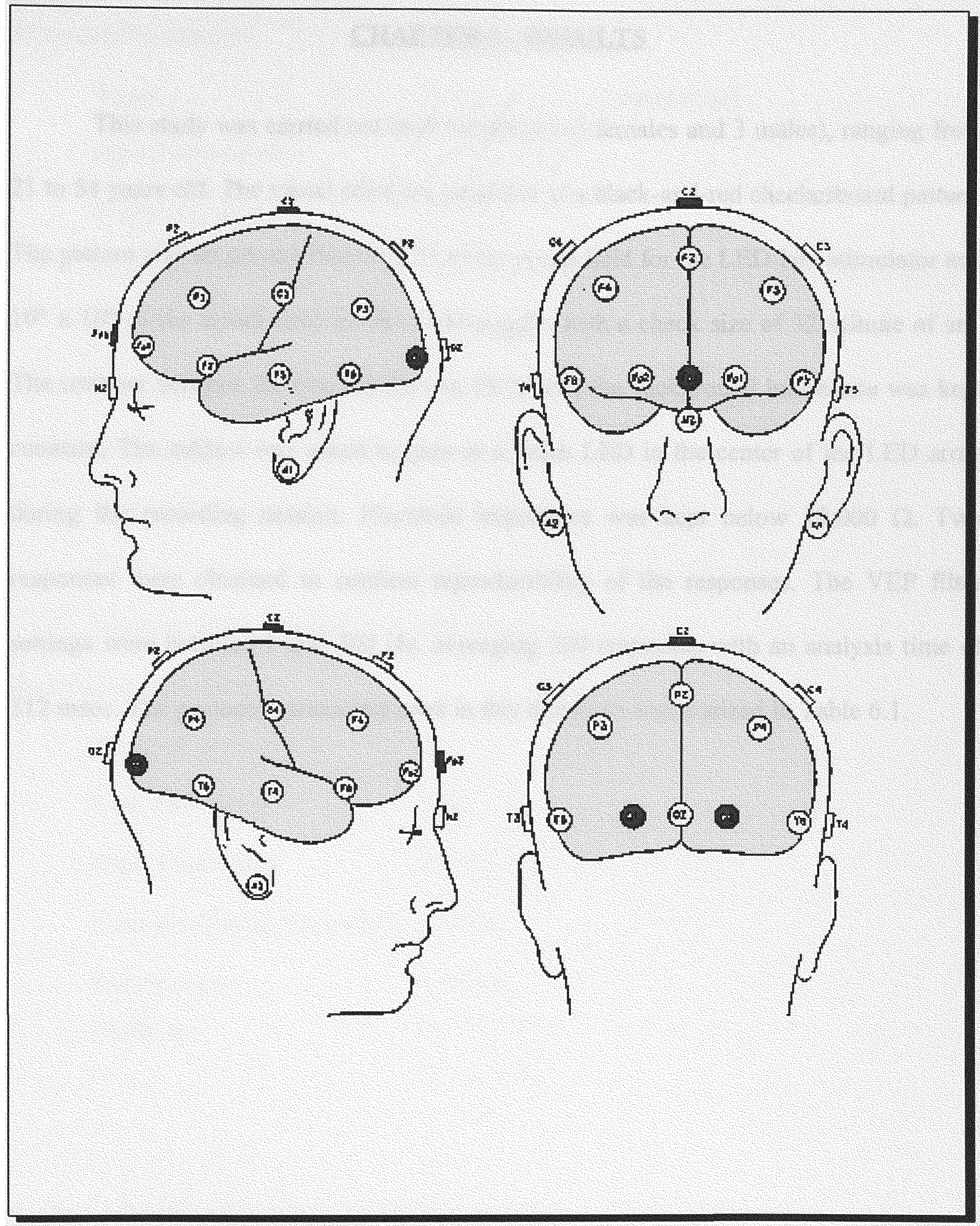

Figure 5.7 Montage of the electrodes in the human's scalp (adapted from Nuwer, 1986). 


\section{CHAPTER 6 - RESULTS}

This study was carried out on 6 volunteers ( 3 females and 3 males), ranging from 21 to 54 years old. The visual stimulus consisted of a black-and-red checkerboard pattern. The pattern was set greater than $9^{\circ} \times 9^{\circ}$ of the visual field for the LED-box stimulator and $10^{\circ} \times 10^{\circ}$ of the visual field for the LED-goggle, with a check size of 32 minute of arc. The contrast between the two checks was $50 \%$, and the background luminance was kept constant. The subject was asked to gaze at a green LED in the center of the LED array during the recording session. Electrode impedance was kept below $10,000 \Omega$. Two responses were obtained to confirm reproducibility of the responses. The VEP filter settings were between 1 and $300 \mathrm{~Hz}$, averaging 200 responses with an analysis time of $512 \mathrm{msec}$. The protocol parameters used in this thesis are summarized in Table 6.1. 
Stimulus Parameters

Type: Checkerboard pattern reversal

Stimulation Rate: $\quad 1$ per second

Sampling time: $\quad 800 \mathrm{usec}$

Viewing distance: $\quad 532$ millimeters for box, 210 millimeters for goggle

Check size: $\quad 32$ minutes of arc

Visual Field $\quad 9^{\circ} \times 9^{\circ}($ Box $), 10^{\circ} \times 10^{\circ}$ (Goggle)

Stimulation: Monocular

Stimulation Type: $\quad$ Full-field

Color: $\quad$ Red

Number of repetitions: $\quad 200$

Recording Parameters

Number of channels: $\quad 2$

Low Pass Filter: $\quad 1 \mathrm{~Hz}$

High Pass Filter: $\quad 300 \mathrm{~Hz}$

Gain (including Pre-Amp): 100,000

Impedances: $\quad<10,000$

Montage: $\quad \mathrm{O}_{1}-\mathrm{C}_{\mathrm{z}}-\mathrm{F}_{\mathrm{pz}}$

$\mathrm{O}_{2}-\mathrm{C}_{\mathrm{z}}-\mathrm{F}_{\mathrm{pz}}$

Table 6.1 Protocol parameters for VEP recordings. 


\subsection{Latency-interpeak amplitude Analysis}

Latencies of P50, N75, P100, and N145 components were measured. The definitions of each VEP components and its amplitude were as follows: P50 was the first positivity; N75 was the first negativity and its amplitude was measured between the peak of N75 and the preceding positivity, P50; P100 was the major positivity, its amplitude was measured between the peak of P100 and its preceding negativity, N75; N145 was the second negativity, its amplitude was measured between the peak of N145 and that of P100. P175 is the third positivity; its amplitude was measured between the peak of 175 and its preceding negativity N145. These definitions follow the standards observed in clinical VEP measurements as shown in Figure 6.1. 
Visual Evoked Potential

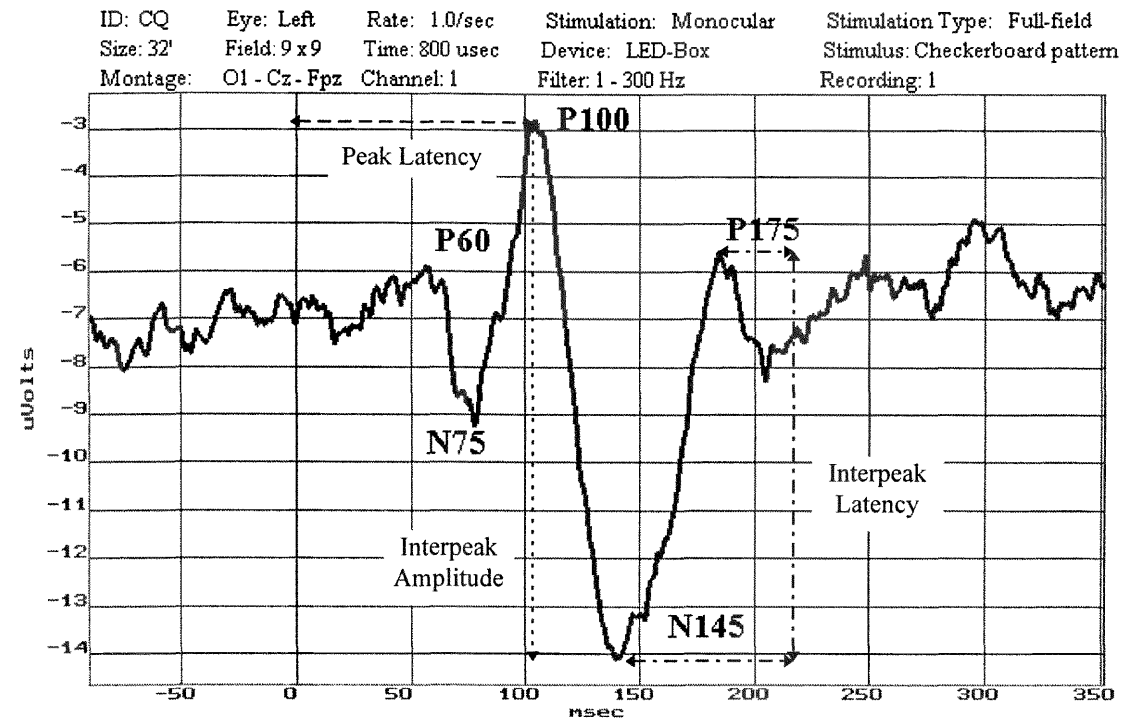

Figure 6.1 How to measure peak latency, interpeak amplitude and latency of a transient VEP.

Subject code and reference values are shown in Table 6.2. The analysis of data was organized as follows: signals collected from each eye of each subject using each stimulator were considered separately (e.g., signals from the left eye of subject "CL" when stimulated with the LED-goggle). There were 200 sweeps in each one of these data sets and they were further divided in four subsets of 50 sweeps each $(1-50,51-100,101$ $150,151-200)$. There were two recording sessions involving both eyes of each subject and stimulation with both the LED-box and the LED-goggle. The mean latencies and interpeak amplitudes measured from each subject appear in Table 6.3, 6.4, 6.5, 6.6, 6.7, 
and 6.8. The 5-character subject code in these tables indicate the subject (CL, CQ, IM, LO, MS, or $\mathrm{RD}$ ), the eye stimulated (L or R), the stimulator used (B or $\mathrm{G}$ ) and the recording session for the subject (1 or 2).

For each subject and recording, the mean latencies are diagrammed in seven figures following the corresponding table (e.g., Fig. 6.3a and 6.4a following table 6.3). Similarly, the interpeak amplitudes are shown in two 3-dimensional graphs following each table. Acquired VEPs, from the LED-box and the LED-goggle, are shown in Fig. 6.2.

\begin{tabular}{|c|c|c|}
\hline $\begin{array}{c}\text { Subject } \\
\text { Code }\end{array}$ & Age & Sex \\
\hline CL & 33 & $\mathrm{M}$ \\
CQ & 54 & $\mathrm{~F}$ \\
IM & 21 & $\mathrm{M}$ \\
LO & 30 & $\mathrm{~F}$ \\
MS & 33 & $\mathrm{~F}$ \\
RD & 31 & $\mathrm{M}$ \\
\hline
\end{tabular}

Table 6.2 Reference values of gathered VEP responses. 

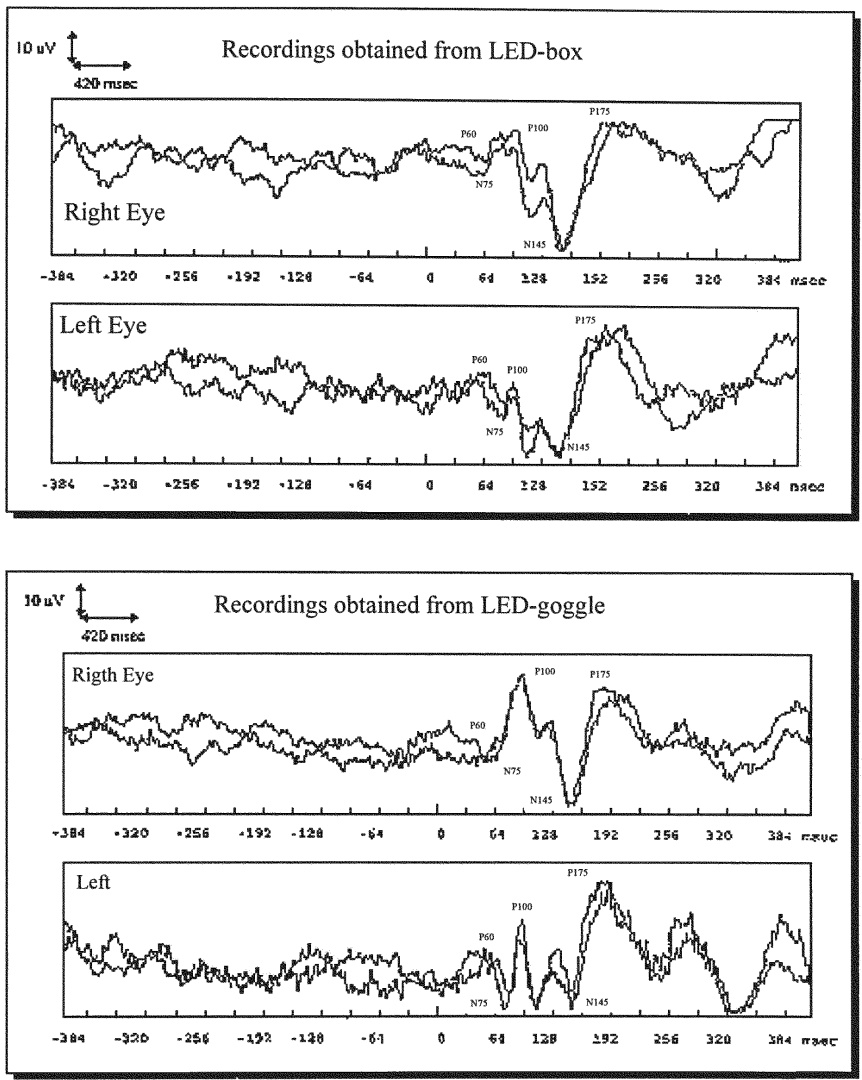

Figure 6.2 Two superimposed recordings from subject RD. Each graph shows the preand post-stimulus of each recording. 


\begin{tabular}{|c|c|c|c|c|c|c|c|c|c|c|}
\hline \multirow{2}{*}{$\begin{array}{l}\text { Syblet } \\
\text { Code }\end{array}$} & \multirow[t]{2}{*}{ Swcopd } & \multirow{2}{*}{$\begin{array}{c}\text { P51 } \\
\text { LAT ms }\end{array}$} & \multirow{2}{*}{$\begin{array}{c}\text { N76 } \\
\text { LAT ms }\end{array}$} & \multicolumn{2}{|c|}{$100-\sin _{1}$ N145 } & \multicolumn{3}{|c|}{ P175 $=$ P50-N75 + N75-P100 } & \multicolumn{2}{|c|}{ P100-N145 N145-P175 } \\
\hline & & & & LAT ms & $\mathrm{LAT} \mathrm{ms}$ & LAT ms & $A M P_{u V}$ & AMP uV & AMP uV & AMP uV \\
\hline \multirow[t]{6}{*}{ CLLBI } & $1-50$ & 51.0 & 79.3 & 116.1 & 145.3 & 184.7 & 3.2 & 5.8 & 7.2 & 14.5 \\
\hline & $51-100$ & 51.0 & 78.3 & 118.7 & 145.3 & 182.1 & 3.9 & 6.6 & 5.1 & 9.4 \\
\hline & $101-150$ & 50.1 & 88.7 & 120.4 & 146.1 & 183.8 & 3.4 & 8.7 & 4.6 & 10.9 \\
\hline & $151-200$ & 48.4 & 88.7 & 124.7 & 153.8 & 183.0 & 5.1 & 10.1 & 3.7 & 8.0 \\
\hline & MEAN & 50.1 & 83.8 & 120.0 & 147.6 & 183.4 & 3.9 & 7.8 & 5.2 & 10.7 \\
\hline & SDT DEV & 1.2 & 5.7 & 3.6 & 4.1 & 1.1 & 0.9 & 2.0 & 1.5 & 2.8 \\
\hline \multirow[t]{6}{*}{ CLRBI } & 1.50 & 47.5 & 70.7 & 115.3 & 138.4 & 183.3 & 2.3 & 2.6 & 1.0 & 3.5 \\
\hline & $51-100$ & 51.0 & 75.8 & 118.7 & 145.3 & 186.4 & 0.4 & 2.7 & 2.4 & 4.4 \\
\hline & $101-150$ & 57.8 & 86.1 & 124.7 & 148.7 & 187,3 & 1.7 & 3.5 & 2.2 & 2.2 \\
\hline & $151-200$ & 63.0 & 913 & 117.8 & 142.7 & 180.4 & 2.6 & 3.0 & 1.6 & 4.1 \\
\hline & MEAN & 54.8 & 81.0 & 119.1 & 143.8 & 184.4 & 1.8 & 3.0 & 1.8 & 3.6 \\
\hline & SDT DEV & 6.9 & 9.4 & 4.0 & 4.3 & 3.1 & 1.0 & 0.4 & 0.6 & 1.0 \\
\hline \multirow[t]{6}{*}{ CLLG1 } & $1-50$ & 49.3 & 85.3 & 109.3 & 140.1 & 171.8 & 2.3 & 2.9 & 1.1 & 1.9 \\
\hline & $51-100$ & 63.8 & 92.1 & 109.3 & 132.4 & 152.1 & 1.4 & 2.8 & 0.8 & 1.2 \\
\hline & $101-150$ & 72.4 & 84.4 & 105.8 & 133.3 & 173.5 & 0.8 & 1.2 & 0.2 & 2.5 \\
\hline & $151-200$ & 53.7 & 80.1 & 99.8 & 129.8 & 173.5 & 0.9 & 0.5 & 1.0 & 3.4 \\
\hline & MEAN & 59.8 & 85.5 & 106.1 & 133.9 & 167.7 & 1.4 & 1.9 & 0.8 & 2.3 \\
\hline & STD DEV & 10.4 & 5.0 & 4.5 & 4.4 & 10.4 & 0.7 & 1.2 & 0.4 & 0.9 \\
\hline \multirow[t]{6}{*}{ CLRG1 } & $1-50$ & 53.5 & 81.8 & 111.0 & 150.4 & 189.8 & 3.6 & 5.7 & 2.2 & 3.5 \\
\hline & $51-100$ & 50.1 & 79.6 & 117.0 & 142.7 & 184.7 & 4.6 & 7.8 & 4.6 & 4.1 \\
\hline & $101-150$ & 54.4 & 80.1 & 122.1 & 149.5 & 170.1 & 2.3 & 7.1 & 1.8 & 3.3 \\
\hline & $151-200$ & 47.5 & 78.4 & 117.0 & 153.0 & 195.8 & 6.0 & 118 & 6.5 & 5.1 \\
\hline & MEAN & 51.4 & 80.0 & 116.8 & 148.9 & 185.1 & 4.1 & 8.1 & 3.8 & 4,0 \\
\hline & STD DEV & 3.2 & 1.4 & 4.5 & 4.4 & 11.0 & 1.6 & 2.6 & 2.2 & 0.8 \\
\hline
\end{tabular}

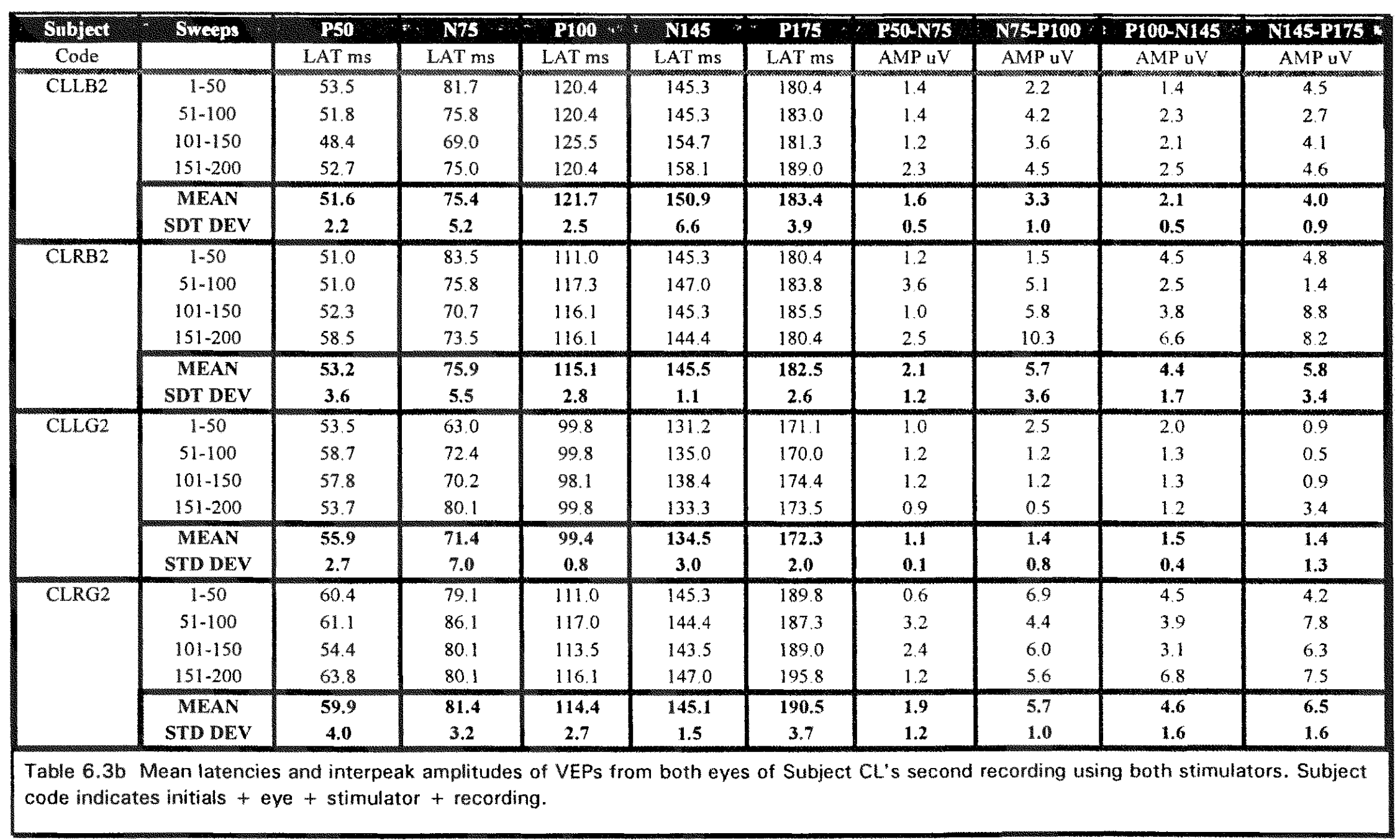


Mean Latencies from first recording - Subject CL

LED-BOX STIMULATOR

Left Eye Right Eye

\section{LED-GOGGLE STIMULATOR}

Left Eye

Right Eye

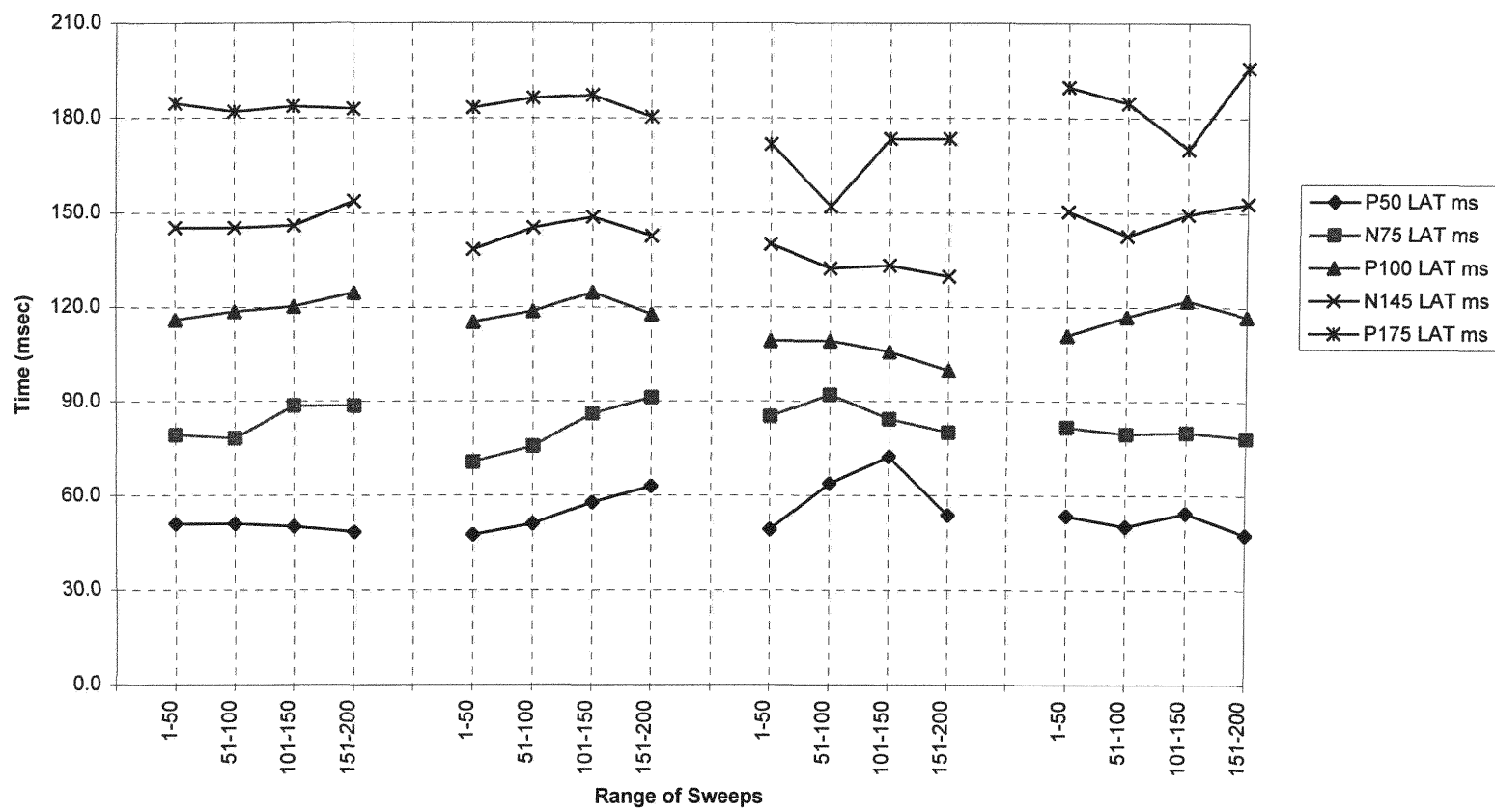

Figure 6.3a Mean latencies in four different ranges of sweeps using both stimulators 


\section{Mean Latencies from second recording - Subject CL}

\section{LED-BOX STIMULATOR}

Left Eye

Right Eye

\section{LED-GOGGLE STIMULATOR}

Left Eye

Right Eye
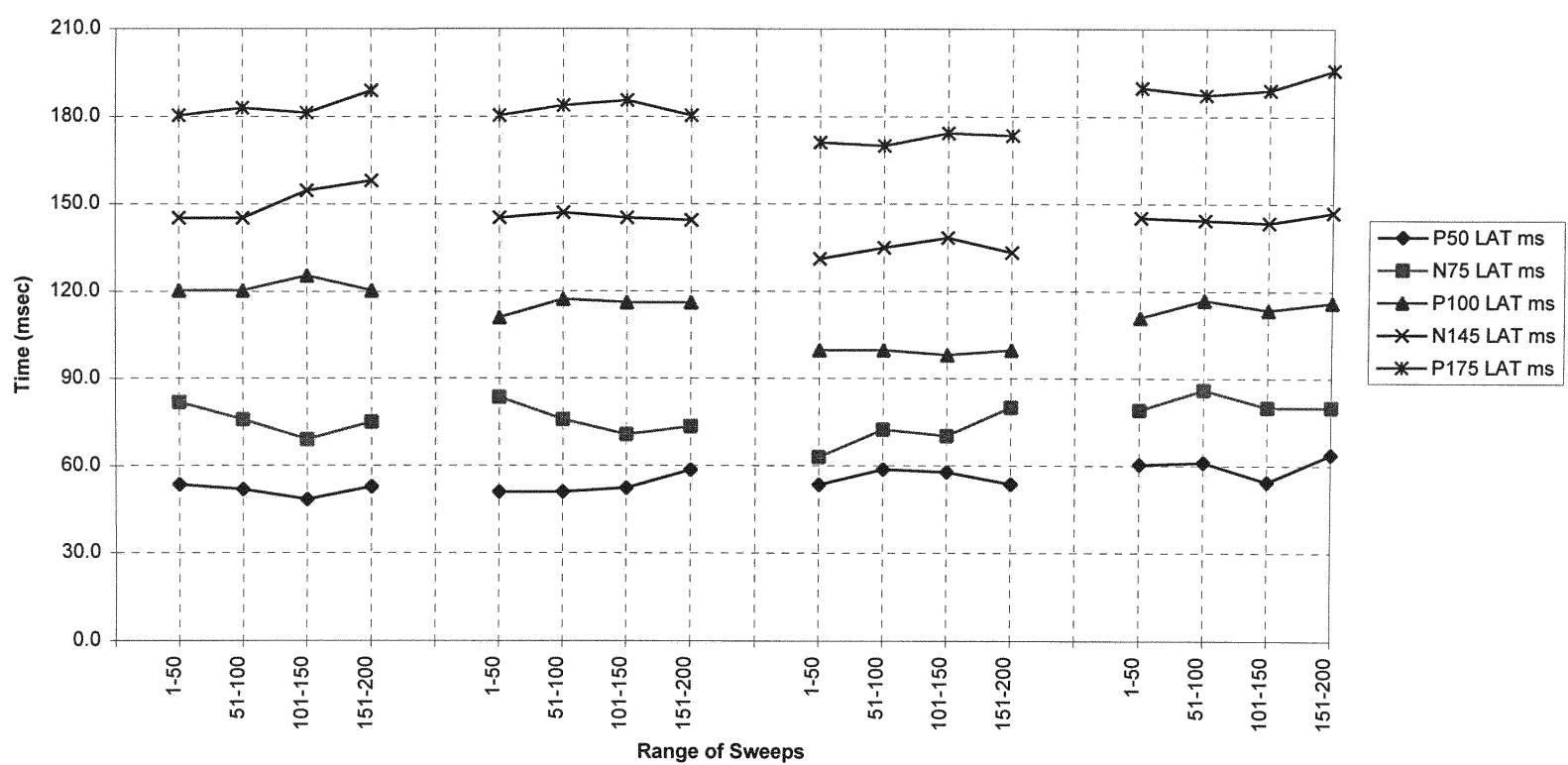

Figure 6.3b Mean latencies in four different ranges of sweeps using both stimulators 


\section{Interpeak Amplitudes from first recording - Subject CL}

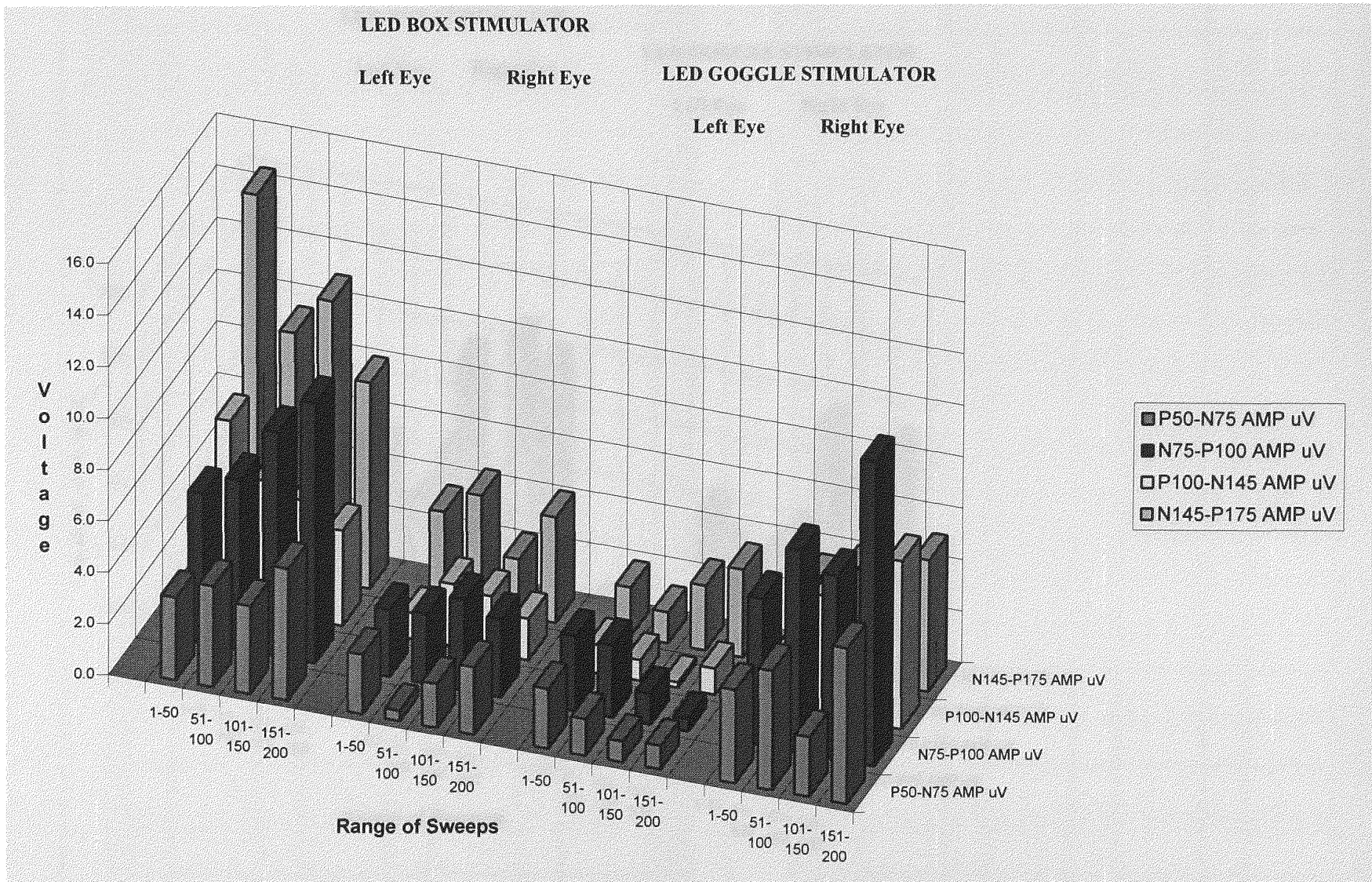

Figure 6.3c Interpeak amplitudes in four different ranges of sweeps using both stimulators 
Interpeak Amplitudes from second recording - Subject CL

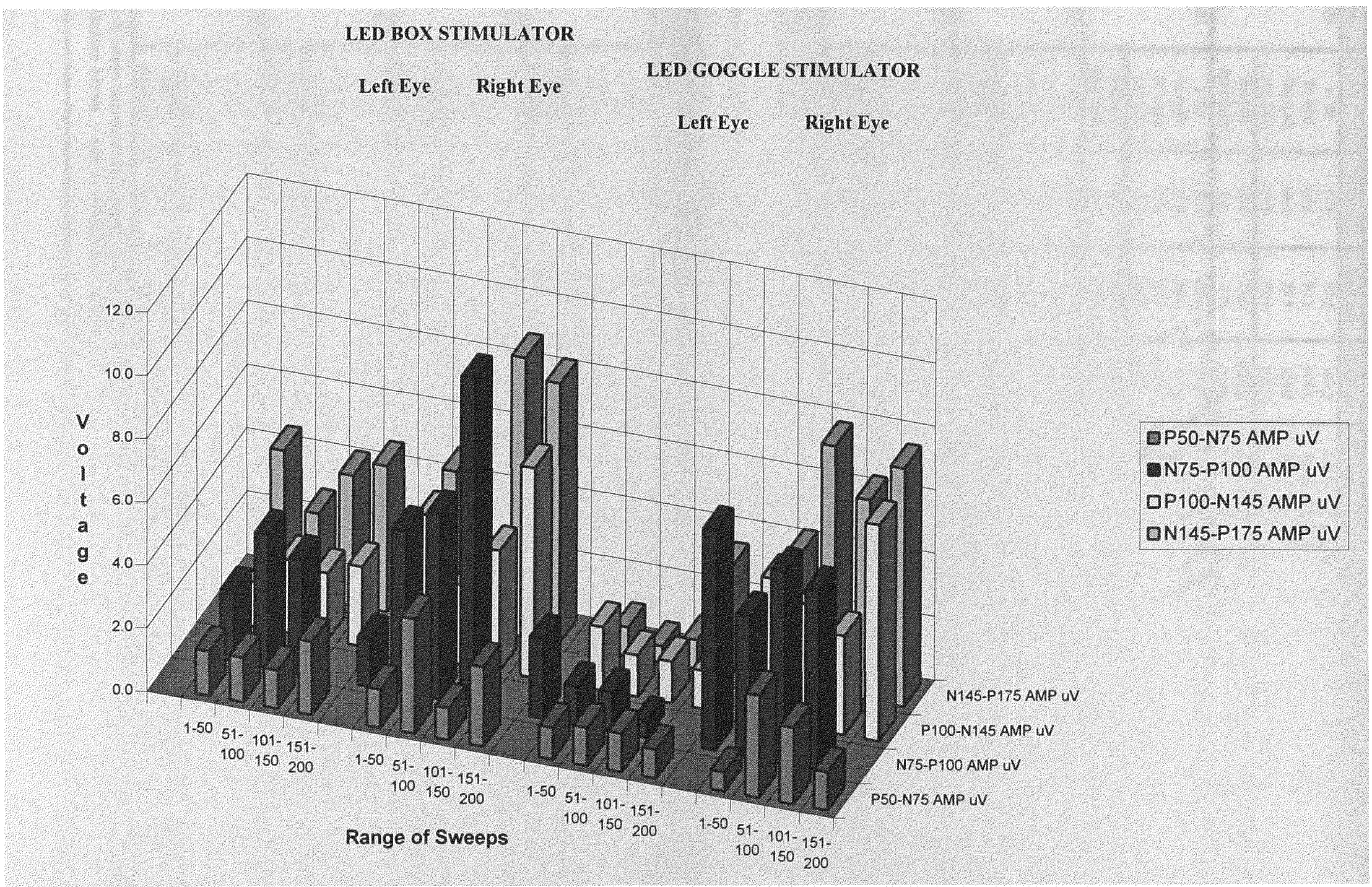

Figure 6.3d Interpeak amplitudes in four different ranges of sweeps using both stimulators 


\begin{tabular}{|c|c|c|c|c|c|c|c|c|c|c|}
\hline \multicolumn{11}{|c|}{ 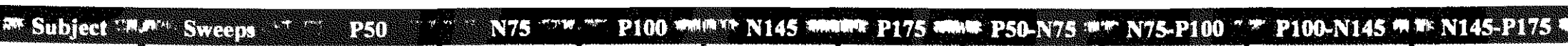 } \\
\hline Code & & LAT $\mathrm{ms}$ & LAT ms & LAT ms & LAT ms & LAT ms & $A M P u V$ & AMP UV & AMP uV & AMP $\mathrm{UV}$ \\
\hline \multirow[t]{6}{*}{ CQLBI } & 1.50 & 57.8 & 77.5 & 103.3 & 141.8 & 186.4 & 6.4 & 9.1 & 6.2 & 6.4 \\
\hline & $51-100$ & 63.8 & 70.7 & 104.1 & 135.8 & 184.7 & 3.3 & 5.5 & 12.1 & 11.0 \\
\hline & $101-150$ & 63.8 & 77.5 & 100.7 & 140.1 & 183.8 & 3.3 & 7.8 & 14.7 & 10.6 \\
\hline & $151-200$ & 57.8 & 72.4 & 105.1 & 135.0 & 187.3 & 3.8 & 8.1 & 116 & 7.6 \\
\hline & MEAN & 60.8 & 74.5 & 103.3 & 138.2 & 185.6 & 4.2 & 7.6 & 11.2 & 8.9 \\
\hline & SDT DEV & 3.5 & 3.5 & 1.9 & 3.3 & 1.6 & 1.5 & 1.5 & 3.6 & 2.3 \\
\hline \multirow[t]{6}{*}{ CQRBI } & 1.50 & 57.8 & 78.4 & 104.0 & 141.0 & 184.0 & 4.6 & 6.4 & 9.2 & 6.4 \\
\hline & $51-100$ & 63.8 & 70.7 & 105.0 & 136.7 & 184.7 & 3.3 & 5.6 & 12.1 & 11.0 \\
\hline & $101-150$ & 63.8 & 77.5 & 100.7 & 140.1 & 184.7 & 3.3 & 7.8 & 14.7 & 10.9 \\
\hline & $151-200$ & 59.5 & 72.4 & 105.8 & 135.8 & 1864 & 3.9 & 8.2 & 11.7 & 7.4 \\
\hline & MEAN & 61.2 & 74.8 & 103.9 & 138.4 & 185.0 & 3.8 & 7.0 & 11.9 & 8.9 \\
\hline & SDT DEV & 3.1 & 3.8 & 2.2 & 2.5 & 1.0 & 0.6 & 1.2 & 2.3 & 2.4 \\
\hline \multirow[t]{6}{*}{ CQLGI } & $1+50$ & 78.1 & 91.3 & 115.3 & 159.0 & 180.0 & 1.8 & 4.0 & 13.0 & 4.4 \\
\hline & $51-100$ & 63.8 & 71.5 & 111.8 & 149.5 & 173.5 & 0.3 & 5.8 & 7.5 & 1.4 \\
\hline & $101-150$ & 56.1 & 81.0 & 111.0 & 157.3 & 184.7 & 3.4 & 5.3 & 9.1 & 3.9 \\
\hline & $151-200$ & 733 & 85.0 & 122.1 & 149.5 & 172.7 & 1.6 & 6.7 & 10.6 & 3.8 \\
\hline & MEAN & 67.8 & 82.2 & 115.1 & 153.8 & 177.7 & 1.8 & 5.5 & 10.1 & 3.4 \\
\hline & STD DEV & 9.8 & 8.3 & 5.1 & 5.0 & 5.7 & 1.3 & 1.1 & 2.3 & 1.3 \\
\hline \multirow[t]{6}{*}{ CQRG! } & $1-50$ & 50.1 & 74.7 & 123.8 & 1564 & 200.1 & 4.8 & 13.9 & 15.8 & 4.0 \\
\hline & $51-100$ & 52.7 & 64.7 & 113.4 & 145.3 & 200.1 & 3.1 & 11.5 & 13.9 & 6.1 \\
\hline & $101-150$ & 51.8 & 69.0 & 117.8 & 162.4 & 189.8 & 1.7 & 11.0 & 14.5 & 2.2 \\
\hline & $151-200$ & 54.4 & 67.0 & 1084 & 167.5 & 199.1 & 2.6 & 13.8 & 18.5 & 7.1 \\
\hline & MEAN & 52.3 & 68.9 & 115.9 & 157.9 & 197.3 & 3.1 & 12.6 & 15.7 & 4.9 \\
\hline & STD DEV & 1.8 & 4.3 & 6.5 & 9.5 & 5.0 & 1.3 & 1.5 & 2.0 & 2.2 \\
\hline \multicolumn{11}{|c|}{$\begin{array}{l}\text { Table } 6.4 \mathrm{a} \text { Mean latencies and interpeak amplitudes of VEPs from both eves of Subject CQ's first recording using both stimulators. Subject } \\
\text { code indicates initials }+ \text { eye }+ \text { stimulator }+ \text { recording. }\end{array}$} \\
\hline \multicolumn{11}{|c|}{ 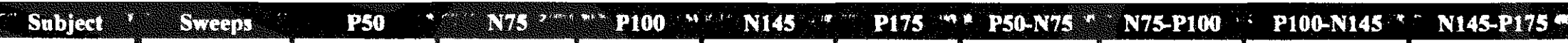 } \\
\hline Code & & LAT ms & LAT $\mathrm{ms}$ & LAT ms & LAT ms & LAT ms & AMP uV & AMP uV & AMP uV & AMP uV \\
\hline \multirow[t]{6}{*}{ CQLB2 } & $1-50$ & 63.8 & 80.1 & 100.7 & 140.1 & 177.8 & 3.3 & 2.2 & 9.8 & 7.0 \\
\hline & $51-100$ & 60.4 & 73.3 & 100.7 & 140.1 & 183.0 & 4.2 & 3.9 & 11.3 & 8.6 \\
\hline & $101-150$ & 63.0 & 78.4 & 102.4 & 140.1 & 180.4 & 2.6 & 5.4 & 11.8 & 6.2 \\
\hline & $151-200$ & 62.1 & 74.1 & 102.4 & 141.0 & 187.3 & 4.6 & 8.4 & 14.8 & 8.8 \\
\hline & MEAN & 62.3 & 76.5 & 101.6 & 140.3 & 182.1 & 3.7 & 5.0 & 11.9 & 7.7 \\
\hline & SDT DEV & 1.5 & 3.3 & 1.0 & 0.5 & 4.1 & 0.9 & 2.6 & 2.1 & 1.3 \\
\hline \multirow[t]{6}{*}{ CQRB2 } & $1-50$ & 63.8 & 72.4 & 105.0 & 141.0 & 177.8 & 2.6 & 10.3 & 16.0 & 7.2 \\
\hline & $51-100$ & 63.0 & 75.8 & 107.5 & 147.0 & 177.0 & 1.2 & 5.6 & 9.5 & 4.2 \\
\hline & $101-150$ & 59.5 & 71.5 & 107.5 & 141.8 & 180.4 & 2.9 & 9.8 & 11.8 & 1.9 \\
\hline & $151-200$ & 58.7 & 71.5 & 107.5 & 151,3 & 184.7 & 3.6 & 13.7 & 193 & 8.2 \\
\hline & MEAN & 61.3 & 72.8 & 106.9 & 145.3 & 180.0 & 2.6 & 9.9 & 14.2 & 5.4 \\
\hline & SDT DEV & 2.5 & 2.0 & 1.3 & 4.8 & 3.5 & 1.0 & 3.3 & 4.4 & 2.9 \\
\hline \multirow[t]{6}{*}{ CQLG2 } & $1-50$ & 69.8 & 95.5 & 120.4 & 141.0 & 181.3 & 2.8 & 4.9 & 3.9 & 1.0 \\
\hline & $51 \cdot 100$ & 66.4 & 88.7 & 111.8 & 148.5 & 180.4 & 3.0 & 6.7 & 8.0 & 1.0 \\
\hline & $101-150$ & 68.1 & 85.3 & 113.5 & 152.1 & 178.7 & 1.1 & 43 & 9.8 & 6.6 \\
\hline & $151-200$ & 69.8 & 92.1 & 111.0 & 152.1 & 184.7 & 2.9 & 4.0 & 5.7 & 4.0 \\
\hline & MEAN & 68.5 & 90.4 & 114.2 & 148.4 & 181.3 & 2.5 & 5.0 & 6.9 & 3.2 \\
\hline & STD DEV & 1.6 & 4.4 & 4.3 & 5.2 & 2.5 & 0.9 & 1.2 & 2.6 & 2.7 \\
\hline \multirow[t]{6}{*}{ CQRG2 } & $1-50$ & 60.3 & 70.7 & 105.0 & 148.7 & 175.3 & 03 & 6.6 & 10.6 & 0.2 \\
\hline & $51-100$ & 53.5 & 70.7 & 102.4 & 144.4 & 171.0 & 0.3 & 7.4 & 9.6 & 0.5 \\
\hline & $101-150$ & 54.4 & 69.1 & 117.0 & 152.1 & 175.3 & 2.0 & 15.6 & 15.8 & 0.6 \\
\hline & $151-200$ & 57.0 & 72.1 & 120.4 & 156.4 & 179.5 & 02 & 9.0 & 14.2 & 2.8 \\
\hline & MEAN & 56.3 & 70.7 & 111.2 & 150.4 & 175.3 & 0.7 & 9.7 & 12.6 & 1.0 \\
\hline & STD DEV & 3.1 & 1.2 & 8.8 & 5.1 & $\mathbf{3 . 5}$ & 0.9 & 4.1 & 2.9 & 1.2 \\
\hline
\end{tabular}




\section{Mean Latencies from first recording - Subject CQ}

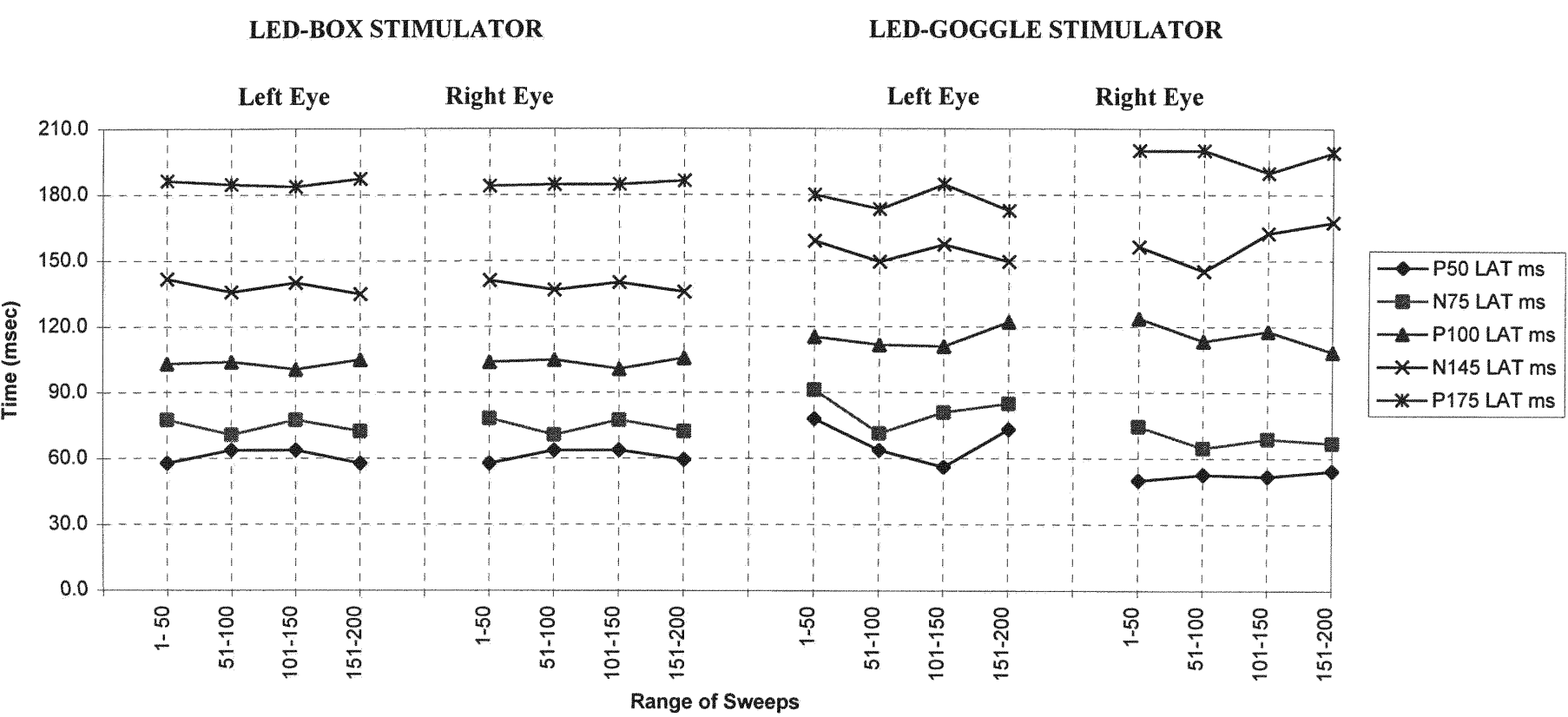

Figure 6.4a Mean latencies in four different ranges of sweeps using both stimulators 


\section{Mean Latencies from second recording - Subject CQ}

\section{LED-BOX STIMULATOR}

LED-BOX STIMULATOR

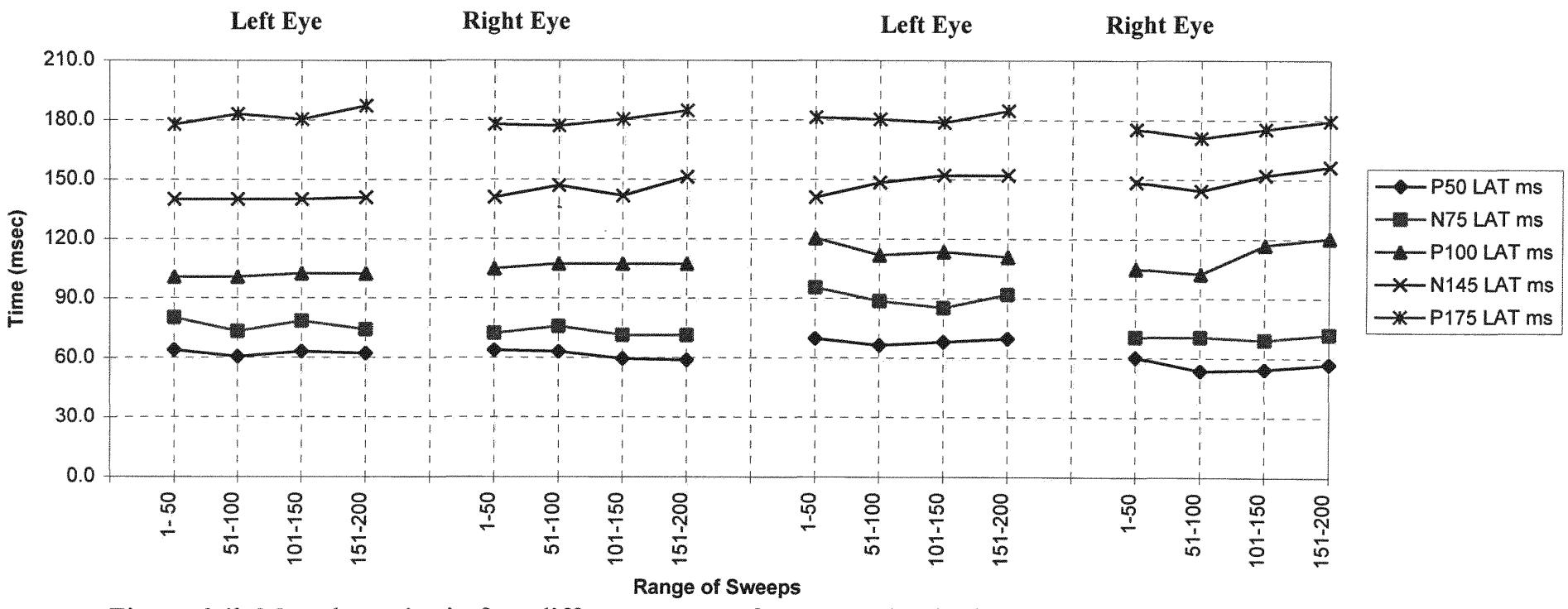

Figure $6.4 \mathrm{~b}$ Mean latencies in four different ranges of sweeps using both stimulators 


\section{Interpeak Amplitude from first recording - Subject CQ}

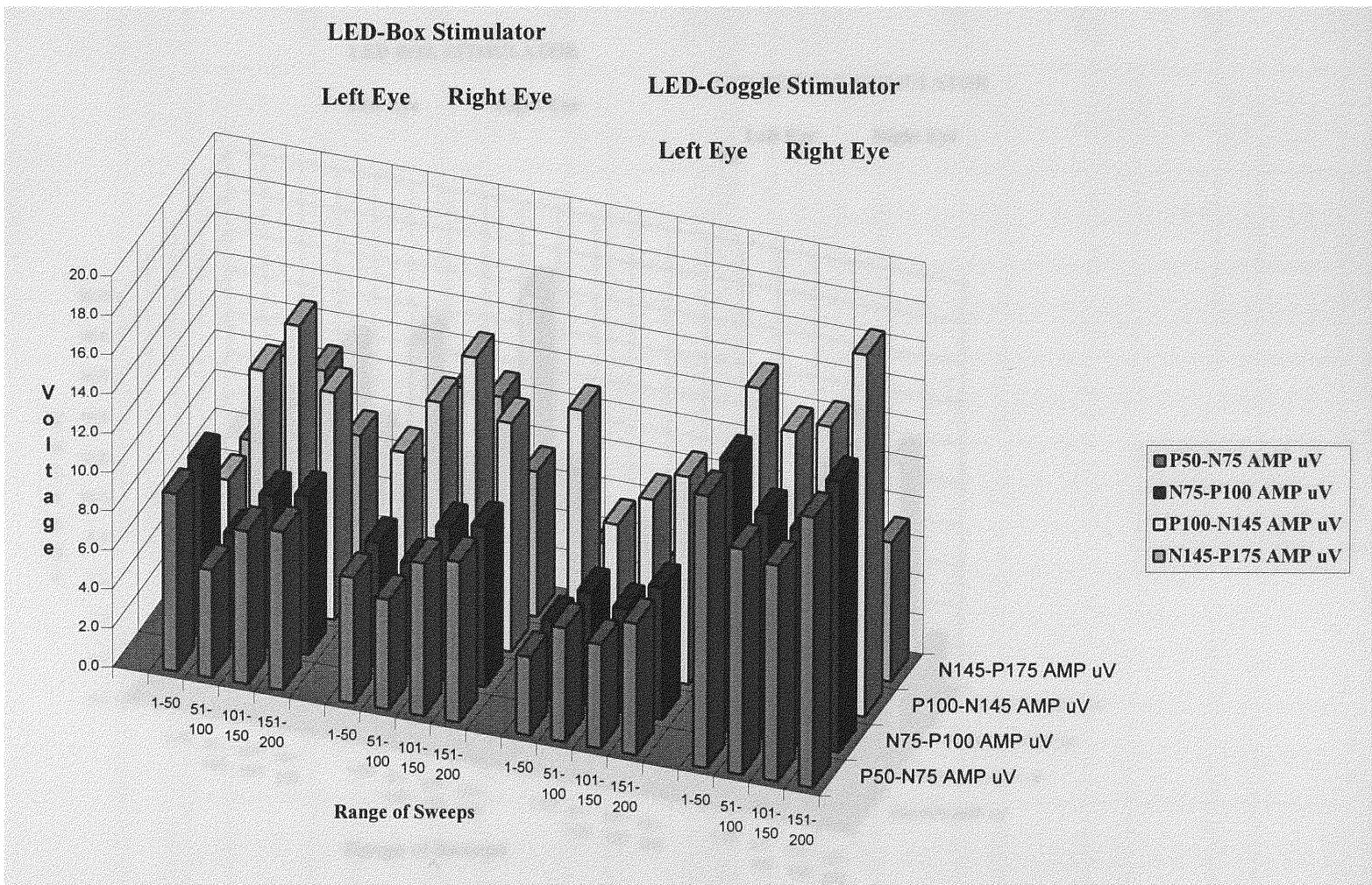

Figure 6.4c Interpeak amplitudes in four different ranges of sweeps using both stimulators 


\section{Interpeak Amplitudes from second recording - Subject CQ}

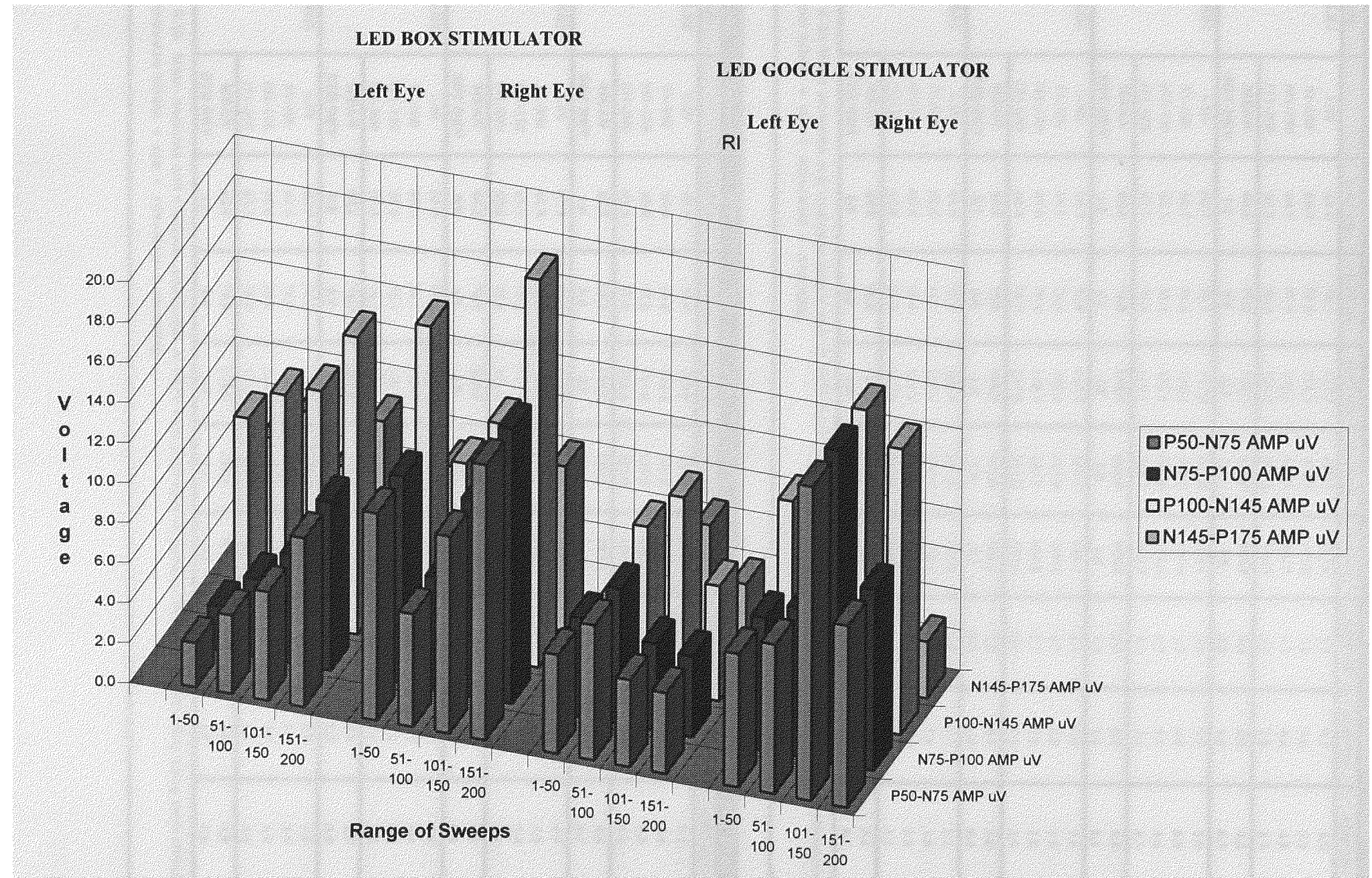

Figure $6.4 \mathrm{~d}$ Interpeak amplitudes in four different ranges of sweeps using both 


\begin{tabular}{|c|c|c|c|c|c|c|c|c|c|c|}
\hline Subject & Sweeps & 150 & N75 & 8100 & N145: & $P 175$ & P50-N76 & N75-P100 & $P 100-N 145$ & N1452175 \\
\hline Code & & LAT $\mathrm{ms}$ & LAT ms & LAT ms & LAT ms & $\mathrm{LAT} \mathrm{ms}$ & $A M P u V$ & AMP uV & $A M P$ UV & $A M P u V$ \\
\hline \multirow[t]{6}{*}{ IMLB I } & $1-50$ & 63.0 & 74.1 & 115,3 & 140.1 & 183.8 & 2.3 & 4.1 & 4,0 & 9.2 \\
\hline & $51-100$ & 64.7 & 73.3 & 116.1 & 136.7 & 179.8 & 1.8 & 3.0 & 3.3 & 7.2 \\
\hline & $101-150$ & 64.7 & 77.5 & 116.1 & 139.3 & 189.0 & 1.3 & 48 & 4.8 & 10.3 \\
\hline & $151-200$ & 65.5 & 78.4 & 119.3 & 141.8 & 190.7 & 3.2 & 5.8 & 4.5 & 8.1 \\
\hline & MEAN & 64.5 & 75.8 & 116.7 & 139.5 & 185.8 & 2.2 & 4.4 & 4.2 & 8.7 \\
\hline & SDT DEV & 1.1 & 2.5 & 1.8 & 2,1 & 5.0 & 0.8 & 1.2 & 0.7 & 1.3 \\
\hline \multirow[t]{6}{*}{ IMRB I } & $1-50$ & 64.7 & 76.7 & 117.8 & 141.8 & 194.1 & 2.6 & 5.3 & 5.0 & 9.2 \\
\hline & $51-100$ & 64.7 & 75.8 & 116.1 & 142.7 & 195.0 & 1.2 & 6.0 & 6.0 & 9.7 \\
\hline & $101-150$ & 65.5 & 78.4 & 122.1 & 141.0 & 193.3 & 1.7 & 4.1 & 3.6 & 12.1 \\
\hline & $151-200$ & 67.3 & 76.4 & 116.1 & 148.7 & 196.7 & 3.7 & 7.0 & 8.5 & 10.1 \\
\hline & MEAN & 65.6 & 76.8 & 118.0 & 143.6 & 194.8 & 2.3 & 5.6 & 5.8 & 10.3 \\
\hline & SDT DEV & 1.2 & 1.1 & 2.8 & 3.5 & 1.5 & 1.1 & 1.2 & 2.1 & 1.3 \\
\hline \multirow[t]{6}{*}{ IMLGI } & $1-50$ & 64.7 & 75.8 & 119.5 & 143.5 & 190.1 & 2.5 & 5.0 & 3.6 & 2.4 \\
\hline & $51-100$ & 65.5 & 75.0 & 1213 & 141.8 & 189.8 & 2.7 & 4.0 & 3.7 & 5.1 \\
\hline & $101-150$ & 65.5 & 75.0 & 118.7 & 140.1 & 189.8 & 2.7 & 5.9 & 3.0 & 4.9 \\
\hline & $151-200$ & 66.4 & 76.7 & 117.8 & 141.8 & 195.0 & 2.0 & 30 & 3.9 & 6.1 \\
\hline & MEAN & 65.5 & 75.6 & 119.3 & 141.8 & 191.2 & 2.5 & 4.5 & 3.6 & 4.6 \\
\hline & STD DEV & 0.7 & 0.8 & 1.5 & 1.4 & 2.6 & 0.3 & 1.3 & 0.4 & 1.6 \\
\hline \multirow[t]{6}{*}{ IMRGI } & $1-50$ & 63.8 & 75.0 & 120.4 & 141.0 & 183.8 & 2.0 & 3.1 & 2.2 & 5.7 \\
\hline & $51-100$ & 63.8 & 74.1 & 117.1 & 137.5 & 188.1 & 3.4 & 5.8 & 3.8 & 8.1 \\
\hline & $101-150$ & 63.8 & 75.0 & 121.3 & 139.5 & 185.5 & 3.7 & 5.1 & 3.2 & 8.5 \\
\hline & $151-200$ & 63.8 & 73.3 & 117.8 & 137.5 & 1884 & 3.7 & 5.8 & 3.5 & 6.9 \\
\hline & MEAN & 63.8 & 74.4 & 119.2 & 138.9 & 186.5 & 3.2 & 5,0 & 3.2 & 7.3 \\
\hline & STD DEV & 0.0 & 0.8 & 2.0 & 1.7 & 2.2 & 0.8 & 1.3 & 0.7 & 1.3 \\
\hline
\end{tabular}

Table 6.5a Mean latencies and interpeak amplitudes of VEPS from both eyes of Subject IM's first recording using both stimulators. Subject code indicates initials + eye + stimulator + recording.

\begin{tabular}{|c|c|c|c|c|c|c|c|c|c|c|}
\hline Subject & Sweeps & P50 & $\overline{N 75}$ & P100 & N145 & P175 & P50-N75 & N75-P100 & P100-N145 & N145-P175 \\
\hline Code & & LAT ms & LAT ms & LAT ms & LAT ms & LAT ms & AMP uV & AMP UV & AMP uV & AMP uV \\
\hline \multirow[t]{6}{*}{ IMLB2 } & $1-50$ & 60.4 & 75.0 & 119.5 & 144.4 & 177.8 & 2.2 & 5.3 & 6.1 & 10.7 \\
\hline & $51-100$ & 63.0 & 75.0 & 114.4 & 141.8 & 183.0 & 2.0 & 6.1 & 5.4 & 10.5 \\
\hline & $101-150$ & 63.8 & 75.8 & 111.0 & 137.5 & 182.1 & 3.4 & 6.2 & 4.8 & 10.1 \\
\hline & $151-200$ & 63.8 & 75.0 & 114.3 & 141.0 & 184.7 & 5.7 & 6.3 & 6.2 & 9.6 \\
\hline & MEAN & 62.8 & 75.2 & 114.8 & 141.2 & 181.9 & 3.3 & 6.0 & 5.6 & 10.2 \\
\hline & SDT DEV & 1.6 & 0.4 & 3.5 & 2.8 & 2.9 & 1.7 & 0.5 & 0.7 & 0.5 \\
\hline \multirow[t]{6}{*}{ IMRB2 } & $1-50$ & 63.8 & 74.1 & 117.0 & 141.0 & 170.1 & 3.2 & 6.3 & 4.4 & 8.1 \\
\hline & $51-100$ & 63.8 & 76.7 & 112.7 & 140.1 & 180.4 & 4.0 & 4.2 & 4.9 & 10.9 \\
\hline & $101-150$ & 65.5 & 73.3 & 117.8 & 141.0 & 178.7 & 2.0 & 3.7 & 3.9 & 9.8 \\
\hline & $151-200$ & 63.8 & 72.4 & 119.5 & 140.1 & 183.8 & 2.9 & 2.6 & 3.4 & 9.3 \\
\hline & MEAN & 64.2 & 74.1 & 116.8 & 140.6 & 178.3 & 3.0 & 4.2 & 4.2 & 9.5 \\
\hline & SDT DEV & 0.9 & 1.9 & 2.9 & 0.5 & 5.8 & 0.8 & 1.6 & 0.6 & 1.2 \\
\hline \multirow[t]{6}{*}{ IMLG2 } & $1-50$ & 63.8 & 75.0 & 118.7 & 141.8 & 189.8 & 2.2 & 5.0 & 2.8 & 6.3 \\
\hline & $51-100$ & 65.5 & 74.1 & 119.5 & 141.0 & 189.8 & 1.5 & 2.8 & 3.4 & 6.2 \\
\hline & $101-150$ & 65.5 & 74.1 & 120.4 & 141.0 & 189.0 & 1.8 & 4.2 & 1.4 & 7.4 \\
\hline & $151-200$ & 65.5 & 74.1 & 120.4 & 140.1 & 187.3 & 2.0 & 5.6 & 2.3 & 5.4 \\
\hline & MEAN & 65.1 & 74.3 & 119.8 & 141.0 & 189.0 & 1.9 & 4.4 & 2.5 & 6.3 \\
\hline & STD DEV & 0.8 & 0.5 & 0.8 & 0.7 & 1.2 & 0.3 & 1.2 & 0.8 & 0.8 \\
\hline \multirow[t]{6}{*}{ IMRG2 } & $1-50$ & 63.0 & 74.1 & 117.0 & 138.4 & 184.7 & 3.6 & 6.8 & 3.3 & 8.2 \\
\hline & $51-100$ & 63.0 & 75.0 & 119.5 & 138.4 & 186.4 & 2.9 & 5.0 & 2.7 & 6.8 \\
\hline & $101-150$ & 63.0 & 75.0 & 119.5 & 137.5 & 189.0 & 2.7 & 3.2 & 4.9 & 7.7 \\
\hline & $151-200$ & 65.5 & 75.0 & 119.5 & 140.1 & 189.8 & 3.1 & 5.1 & 4.4 & 6.6 \\
\hline & MEAN & 63.6 & 74.8 & 118.9 & 138.6 & 187.5 & 3.1 & 5.0 & 3.8 & 7.3 \\
\hline & STD DEV & 1.3 & 0.4 & 1.3 & 1.1 & 2.4 & 0.4 & 1.5 & 1.0 & 0.8 \\
\hline
\end{tabular}


Mean Latencies from first recording - Subject IM

LED-BOX STIMULATOR

Left Eye Right Eye

\section{LED-GOGGLE STIMULATOR}

Left Eye

Right Eye
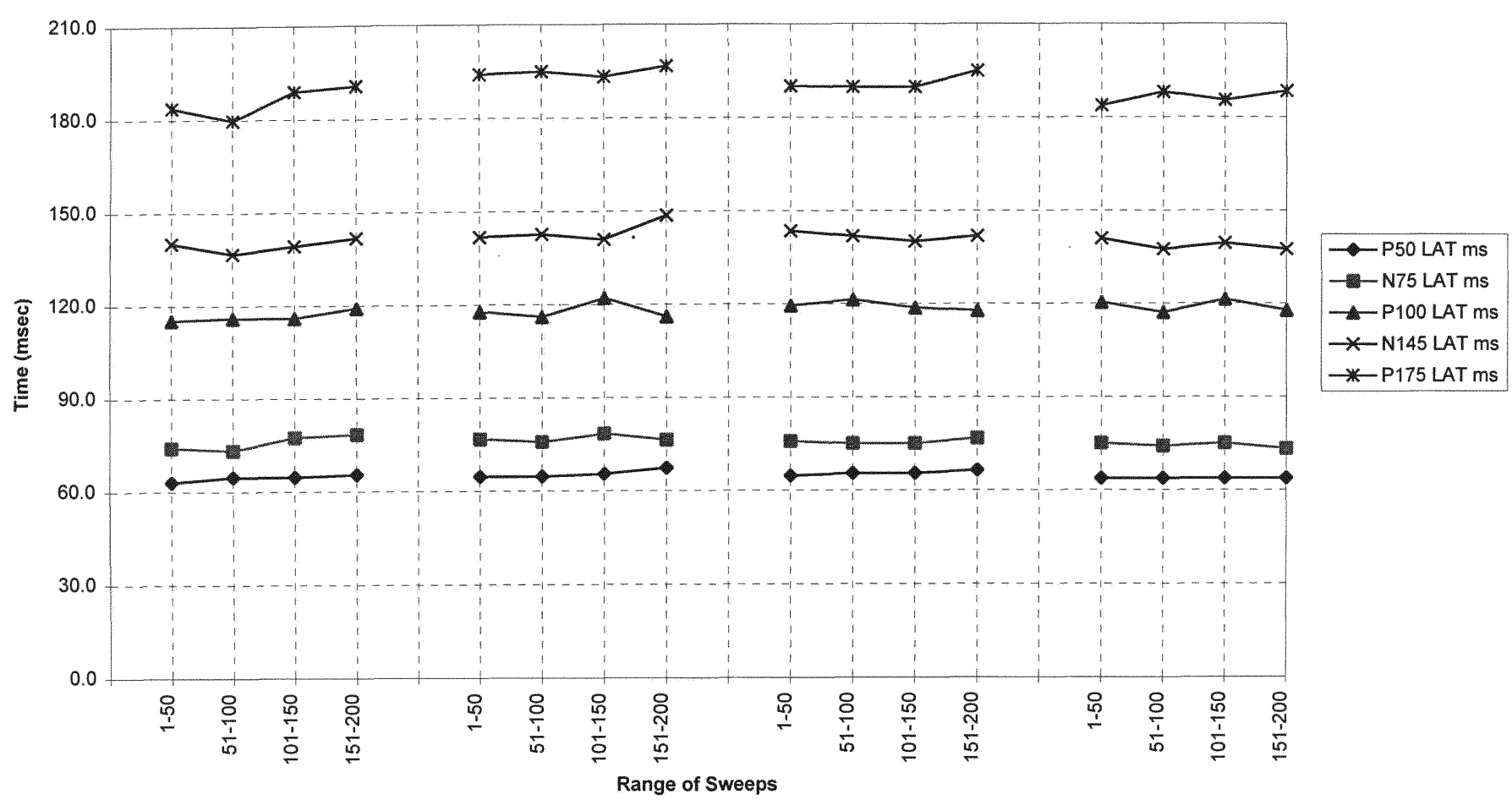

Figure 6.5a Mean latencies in four different ranges of sweeps using both stimulators 
Mean Latencies from second recording - Subject IM

\section{LED-BOX STIMULATOR}

Left Eye

Right Eye

\section{LED-GOGGLE STIMULATOR}

Left Eye

Right Eye
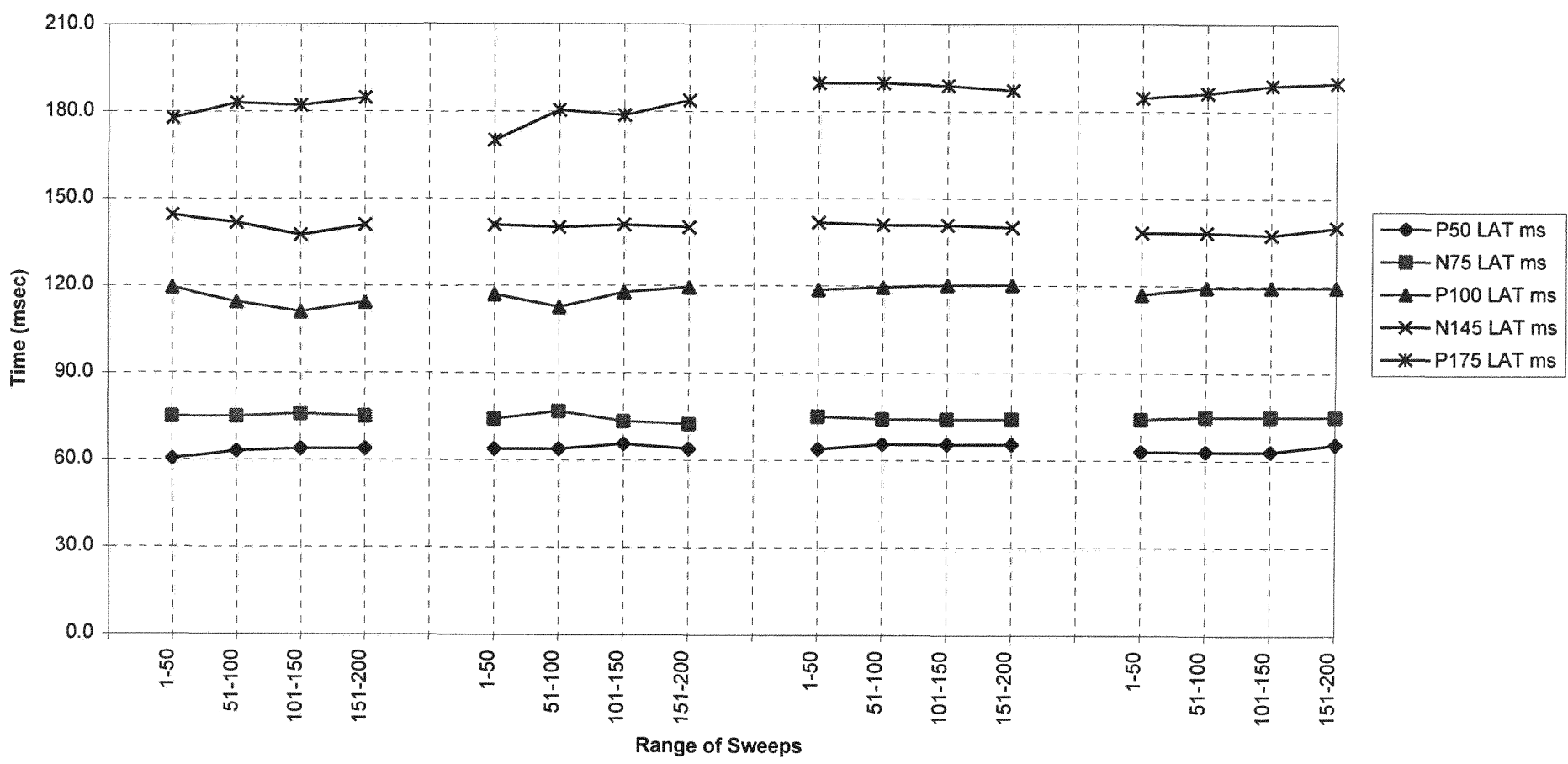

Figure $6.5 \mathrm{~b}$ Mean latencies in four different ranges of sweeps using both stimulators 
Interpeak Amplitudes from first recording - Subject IM

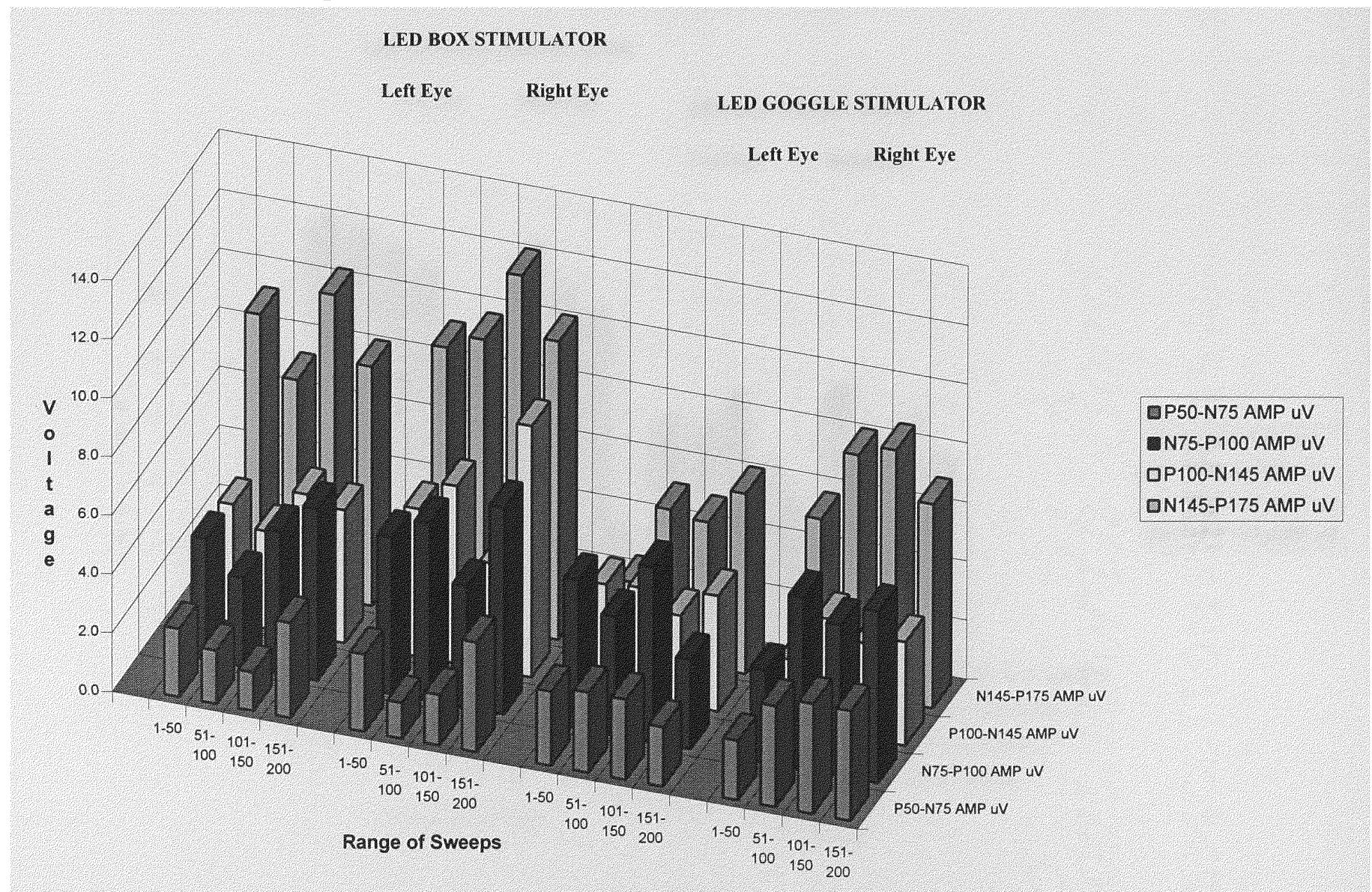

Figure $6.5 \mathrm{c}$ Interpeak amplitudes in four different ranges of sweeps using both stimulators 


\section{Interpeak Amplitudes from second recording - Subject IM}

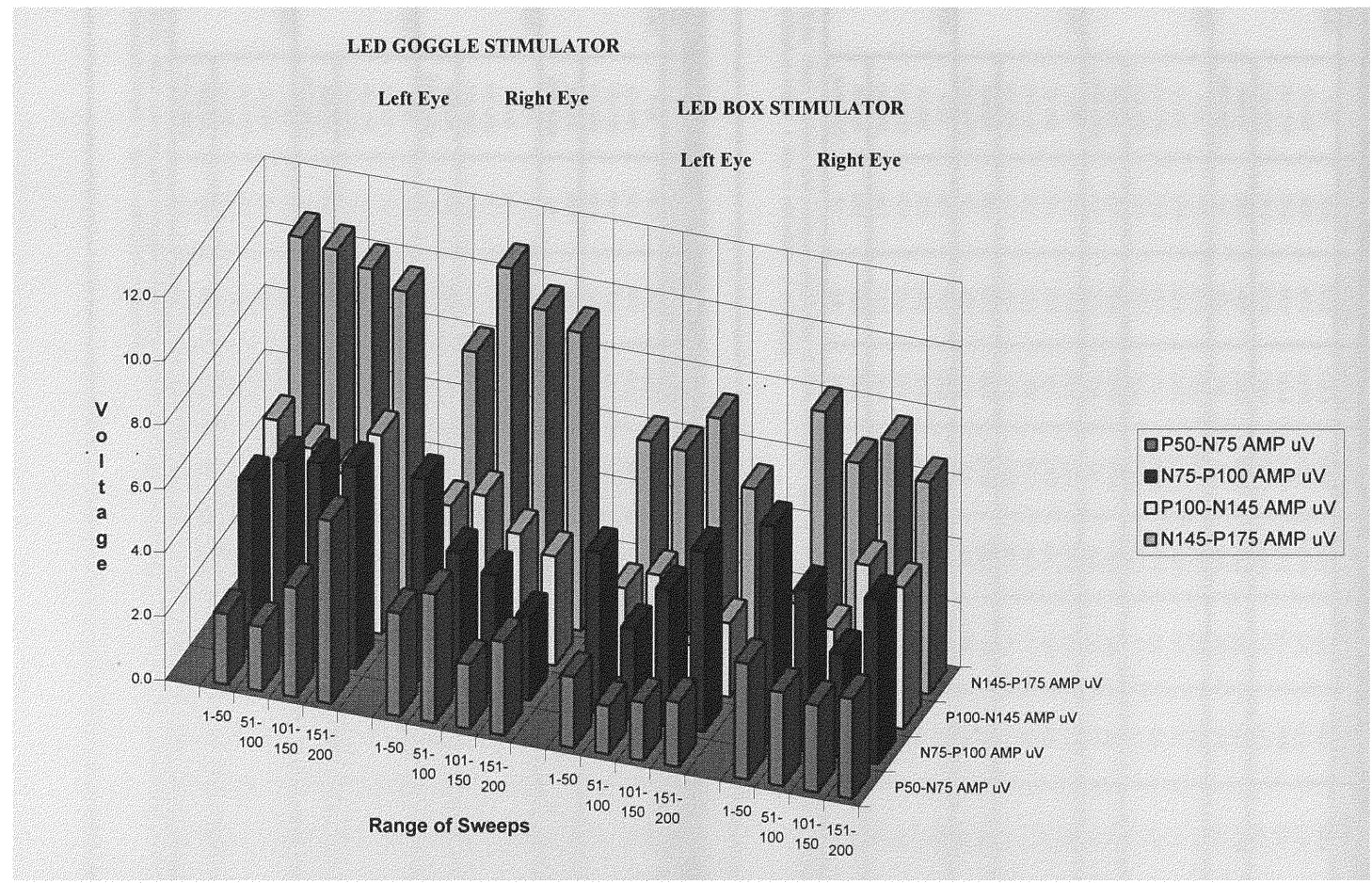

Figure 6.5d Interpeak amplitudes in four different ranges of sweeps using both stimulators 


\begin{tabular}{|c|c|c|c|c|c|c|c|c|c|c|}
\hline \multicolumn{2}{|c|}{ Subject } & 260 & \multicolumn{2}{|c|}{ 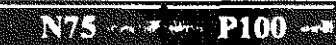 } & \multicolumn{6}{|c|}{ 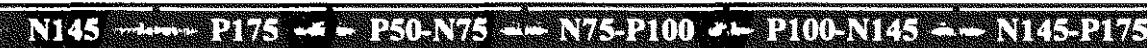 } \\
\hline Code & 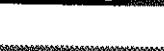 & LAT $\mathrm{ms}$ & LAT ms & LAT $\mathrm{ms}$ & LAT ms & LAT ms & AMP uV & AMP uV & AMP UV & $\mathrm{AMP} u \mathrm{~V}$ \\
\hline \multirow[t]{6}{*}{ LOLBI } & $1-50$ & 59.5 & 83.3 & 115.3 & 155.5 & 165.0 & 13.0 & 16.0 & 25.0 & 6.2 \\
\hline & $51-100$ & 59.5 & 83.1 & 111.8 & 159.0 & 183.0 & 5.3 & 11.4 & 21.5 & 5.7 \\
\hline & $101-150$ & 57.0 & 85.3 & 111.8 & 141.0 & 177.0 & 13.1 & 10,8 & 10.0 & 4.4 \\
\hline & $151-200$ & 59.5 & 87.8 & 120.4 & 153.0 & 192.0 & 79 & 10.7 & 15.1 & 29 \\
\hline & MEAN & 58.9 & 84.9 & 114.8 & 152.1 & 179.3 & 9.8 & 12.2 & 17.9 & 4.8 \\
\hline & SDT DEV & 1.3 & 2.2 & 4.1 & 7.8 & 11.3 & 3.9 & 2.5 & 6.7 & 1.5 \\
\hline \multirow[t]{6}{*}{ LORBI } & 1.50 & 51.0 & 85.3 & 128.1 & 152.1 & 159.0 & 12.8 & 8.8 & 7.6 & 8.5 \\
\hline & $51-100$ & 53.5 & 89.5 & 112.7 & 153.0 & 163.0 & 6.7 & 7.2 & 7.7 & 5.4 \\
\hline & $101-150$ & 55.3 & 92.1 & 113.5 & 153.8 & 177.3 & 9.2 & 9.8 & 109 & 11.4 \\
\hline & $151-200$ & 52.7 & 93.7 & 122.1 & 150,4 & 181.3 & 7.1 & 6.3 & 53 & 69 \\
\hline & MEAN & 53.1 & 90.2 & 119.1 & 152.3 & 170.2 & 9.0 & 8.0 & 7.9 & 8.1 \\
\hline & SDT DEV & 1.8 & 3.7 & 7.4 & 1.5 & 10.8 & 2.8 & 1.6 & 2,3 & 2.6 \\
\hline \multirow[t]{6}{*}{ LOLGI } & 1.50 & 50.1 & 71.4 & 89.5 & 126.3 & 189.8 & 5.4 & 7.5 & 9.6 & 23.1 \\
\hline & $51-100$ & 50.1 & 72.4 & 94.7 & 129.8 & 194.1 & 4.0 & 8.0 & 12.5 & 20.1 \\
\hline & $101-150$ & 50.1 & 72.4 & 90.2 & 127.3 & 174.4 & 5.2 & 10.9 & 7.2 & 13.0 \\
\hline & $151-200$ & 61.3 & 70.7 & 93.8 & 135.0 & 180.1 & 4.7 & 11.3 & 140 & 16.0 \\
\hline & MEAN & 52.9 & 71.7 & 92.1 & 129.6 & 184.6 & 4.8 & 9.4 & 10.8 & 18.1 \\
\hline & STD DEV & 5.6 & 0.8 & 2.6 & 3.9 & 9.0 & 0.6 & 2.0 & 3.0 & 4.5 \\
\hline \multirow[t]{6}{*}{ LORGI } & $1-50$ & 56.1 & 72.5 & 93.0 & 1350 & 168.5 & 91 & 8.5 & 12.9 & 18.1 \\
\hline & $51-100$ & 52.7 & 63.8 & 91.5 & 124.7 & 159.8 & 11.6 & 18.5 & 15.2 & 14.1 \\
\hline & $101-150$ & 63.0 & 73,3 & 92.1 & 135.0 & 161.5 & 3.8 & 17.7 & 16.9 & 17.1 \\
\hline & $151-200$ & 64.7 & 77.5 & 93.0 & 136.7 & 159.8 & 5.3 & 9.0 & 12.5 & 15.4 \\
\hline & MEAN & 59.1 & 71.8 & 92.4 & 132.9 & 162.4 & 7.5 & 13.4 & 14.4 & 16.2 \\
\hline & STD DEV & 5.7 & 5.8 & 0.7 & 5.5 & 4.1 & 3.6 & 5.4 & 2.1 & 1.8 \\
\hline
\end{tabular}

Table 6.6a Mean latencies and interpeak amplitudes of VEPs from both eyes of Subject LO's first recording using both stimulators. Subject code indicates initials + eye + stimulator + recording.

\begin{tabular}{|c|c|c|c|c|c|c|c|c|c|c|}
\hline \multirow{2}{*}{$\begin{array}{c}\text { S. Subject } \\
\text { Code }\end{array}$} & \multirow[t]{2}{*}{ Swcops } & 180 & \multicolumn{2}{|c|}{ N6- $-2=8100-4$} & \multicolumn{3}{|c|}{ N145- $=$ P175 - E PSO-N75 } & \multirow{2}{*}{$\begin{array}{l}\text { N75P100 } \\
\text { AMP UV }\end{array}$} & P100-N145: & \multirow{2}{*}{$\begin{array}{l}\text { N165 P175 } \\
\text { AMP uV }\end{array}$} \\
\hline & & LAT ms & LAT ms & $\mathrm{LAT} \mathrm{ms}$ & LAT ms & LAT ms & $A M P U V$ & & AMP UV & \\
\hline \multirow[t]{6}{*}{ LOLB2 } & $1-50$ & 56.1 & 76.7 & 124.7 & 137.5 & 165.0 & 11.4 & 6.9 & 9.4 & 0.9 \\
\hline & $51-100$ & 62.1 & 80.1 & 1213 & 144.4 & 158.1 & 5.6 & 7,0 & 5.8 & 0.8 \\
\hline & $101-150$ & 56.1 & 72.4 & 121.3 & 143.5 & 177.8 & 7.1 & 22 & 3.1 & 3.3 \\
\hline & $151-200$ & 58.4 & 75.0 & 126.4 & 145.3 & 175.3 & 7.7 & 1.3 & 58 & 79 \\
\hline & MEAN & 58.2 & 76.1 & 123.4 & 142.7 & 169.1 & 8.0 & 4.4 & 6.0 & 3.2 \\
\hline & SDT DEV & 2.8 & 3.2 & 2.5 & 3.5 & 9.2 & 2.5 & 3.0 & 2.6 & $\mathbf{3 . 3}$ \\
\hline \multirow[t]{6}{*}{ LORB2 } & $1-50$ & 51.0 & 84.4 & 126.4 & 141.0 & 175.3 & 13.3 & 8.8 & 78 & 1.7 \\
\hline & $51-100$ & 54.4 & 79.8 & 115.3 & 146.1 & 177.0 & 6.1 & 6.4 & 7.4 & 4.4 \\
\hline & $101-150$ & 55,3 & 98.5 & 114.4 & 145.3 & 178.7 & 8.9 & 9.7 & 5.6 & 6.1 \\
\hline & $151-200$ & 54.4 & 71.5 & 117.0 & 142.7 & 175.3 & 5.8 & 4.6 & 3.8 & 0.3 \\
\hline & MEAN & 53.8 & 83.6 & 118.3 & 143.8 & 176.6 & 8.5 & 7.4 & 6.2 & 3.1 \\
\hline & SDT DEV & 1.9 & 11.3 & 5.5 & 2.4 & 1.6 & 3.5 & 2.3 & 1.8 & 2.6 \\
\hline \multirow[t]{6}{*}{ LOLG2 } & $1-50$ & 51.8 & 71.5 & 90.4 & 114.4 & 195.8 & 3.3 & 8.1 & 7.1 & 20.4 \\
\hline & $51-100$ & 55.3 & 74.1 & 92.1 & 126.4 & 174.4 & 3.3 & 5.1 & 6.2 & 16.4 \\
\hline & $101-150$ & 55.3 & 75.8 & 90.4 & 124.7 & 174.4 & 6.0 & 5.3 & 5.7 & 18.0 \\
\hline & $151-200$ & 57.8 & 70.7 & 93.8 & 131.5 & 194.1 & 0.4 & 16.4 & 19.1 & 22.7 \\
\hline & MEAN & 55.1 & 73.0 & 91.7 & 124.3 & 184.7 & 3.3 & 8.7 & 9.5 & 19.4 \\
\hline & STD DEV & 2.5 & 2.4 & 1.6 & 7.2 & 11.9 & 2.3 & 5.3 & 6.4 & 2.8 \\
\hline \multirow[t]{6}{*}{ LORG2 } & $1-50$ & 51.0 & 75.8 & 101.5 & 137.5 & 179.8 & 4.7 & 9.8 & 10.7 & 2.2 \\
\hline & $51-100$ & 50.1 & 74.1 & 106.7 & 144.4 & 175.3 & 6.6 & 9.0 & 8.2 & 2.1 \\
\hline & $101-150$ & 53.5 & 75.0 & 106.7 & 137.5 & 173.5 & 3.2 & 3.9 & 7.7 & 3.6 \\
\hline & $151-200$ & 52.7 & 77.5 & 114.4 & 147.0 & 178.5 & 2.5 & 7.4 & 8,0 & 7.0 \\
\hline & MEAN & 51.8 & 75.6 & 107.3 & 141.6 & 176.8 & 4.3 & 7.5 & 8.7 & 3.7 \\
\hline & STD DEV & 1.6 & 1.4 & 5.3 & 4.9 & 2.9 & 1.8 & 2.6 & 1.4 & 2.3 \\
\hline
\end{tabular}

Table 6.6b Mean latencies and interpeak amplitudes of VEPS from both eves of Subject LO's second recording using both stimulators. Subject code indicates initials + eye + stimulator + recording. 


\section{Mean Latencies from first recording - Subject LO}

\section{LED-BOX STIMULATOR}

Left Eye

Right Eye

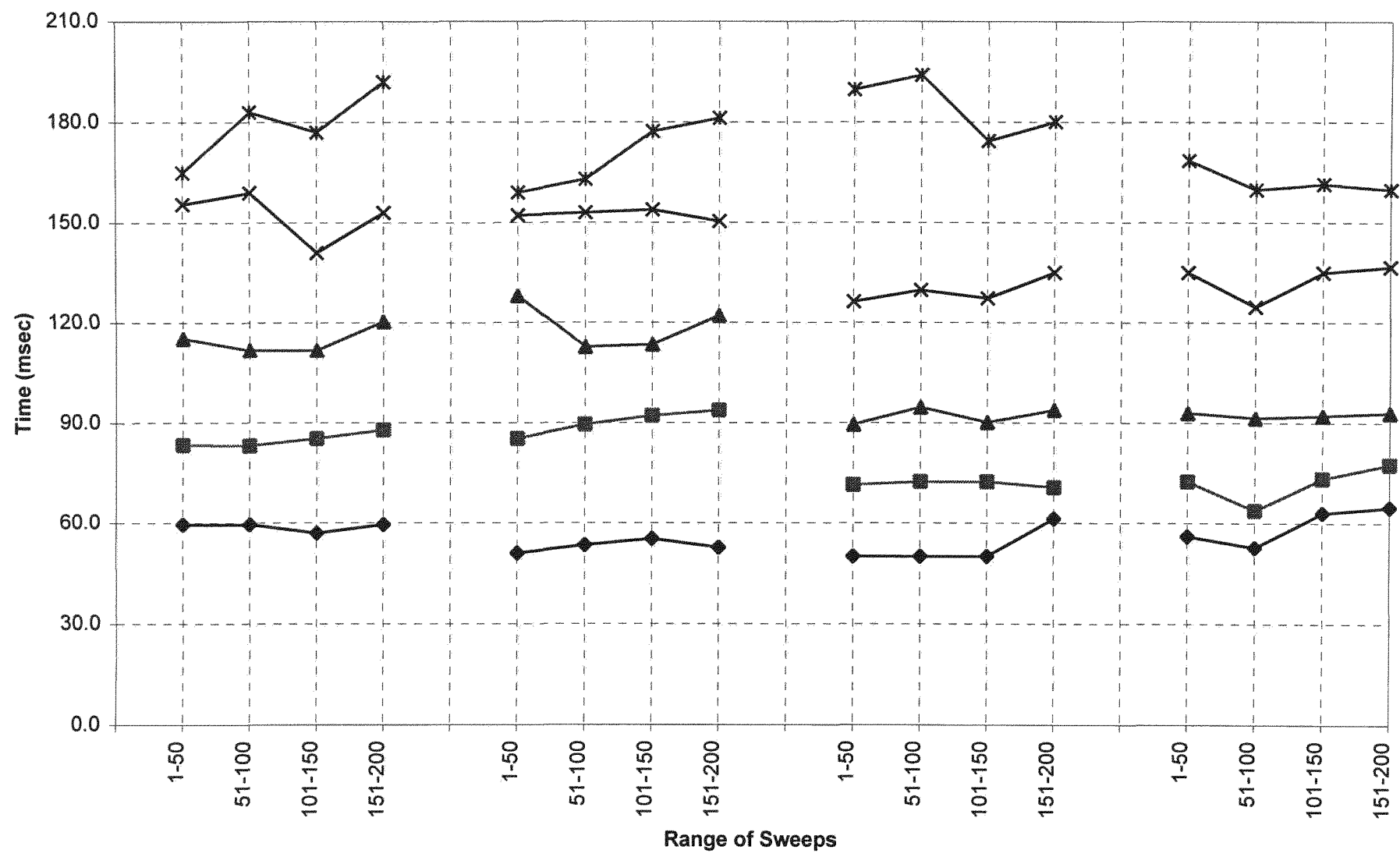

\section{LED-GOGGLE STIMULATOR}

Left Eye

Right Eye
2

Range of Sweeps

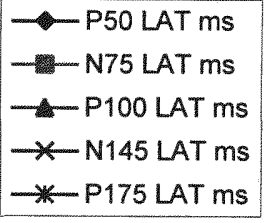

Figure 6.6a Mean latencies in four different ranges of sweeps using both stimulators 


\section{Mean Latencies from second recording - Subject LO}

\section{LED-BOX STIMULATOR}

Left Eye

Right Eye
LED-GOGGLE STIMULATOR

Left Eye Right Eye

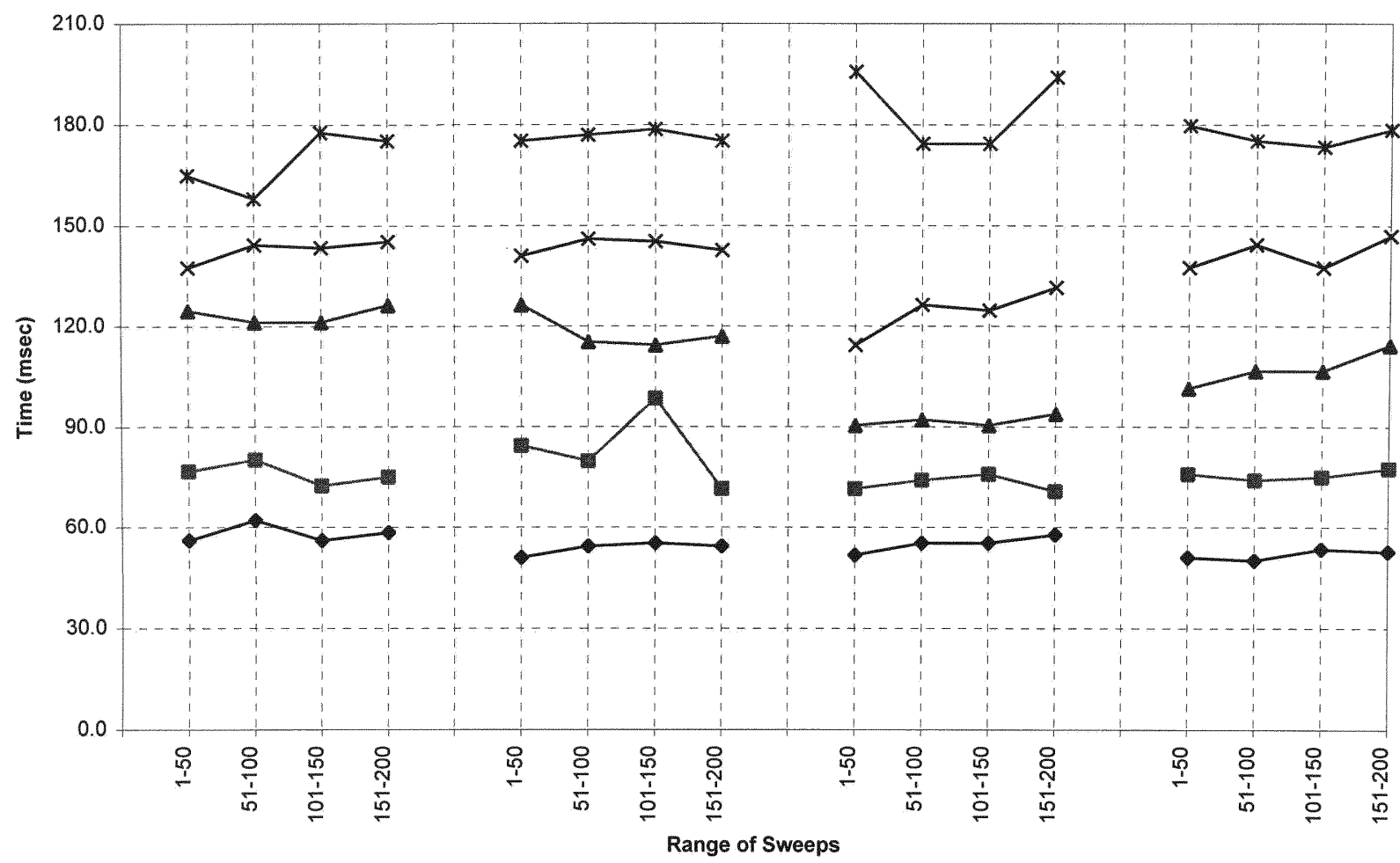

Figure 6.6b Mean latencies in four different ranges of sweeps using both stimulators 


\section{Interpeak Amplitudes from second recording - Subject LO}

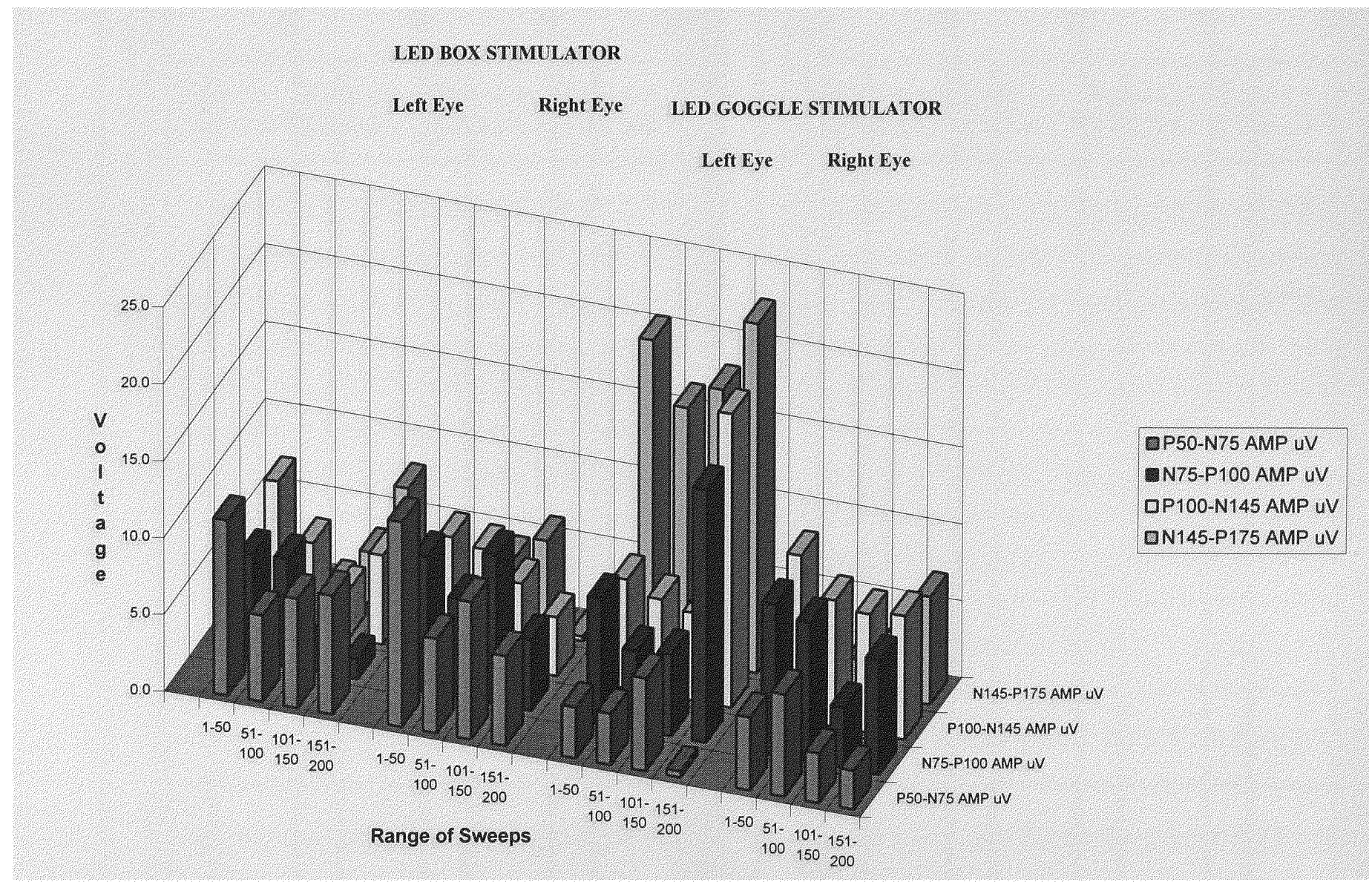

Figure 6.6d Interpeak amplitudes in four different ranges of sweeps using both 


\section{Interpeak Amplitudes from second recording - Subject LO}

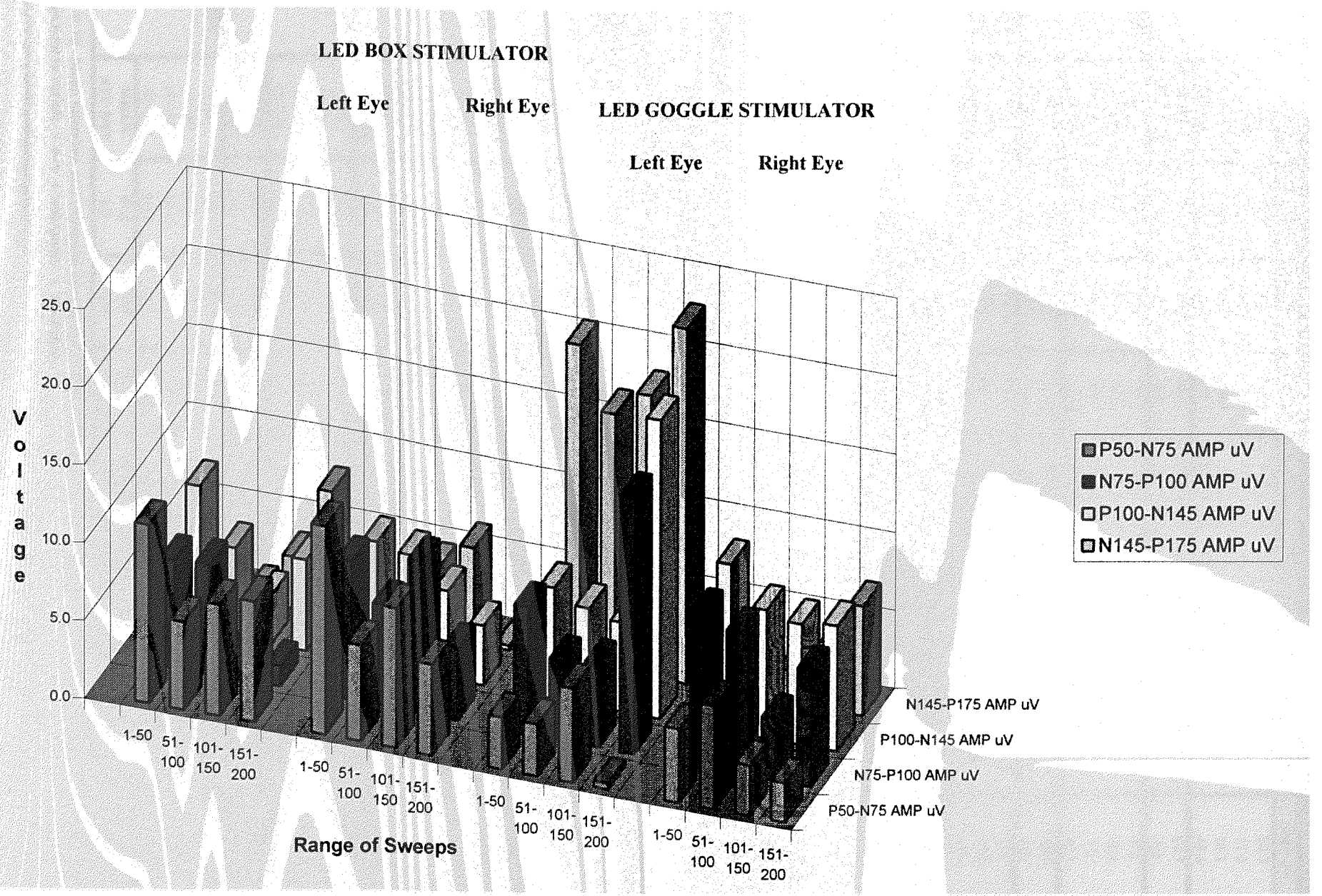

Figure 6.6d Interpeak amplitudes in four different ranges of sweeps using both 


\begin{tabular}{|c|c|c|c|c|c|c|c|c|c|c|}
\hline \multirow{2}{*}{$\begin{array}{l}\text { Srouge } \\
\text { Code }\end{array}$} & \multirow[t]{2}{*}{ Swceps } & \multirow{2}{*}{$\begin{array}{c}\mathrm{P} 50 \\
\mathrm{LAT} \mathrm{ms}\end{array}$} & \multirow{2}{*}{$\begin{array}{l}\text { N76 } \\
\text { LAT ms }\end{array}$} & \multirow{2}{*}{$\begin{array}{l}\text { TOTO } \\
\text { LAT ms }\end{array}$} & \multicolumn{6}{|c|}{ 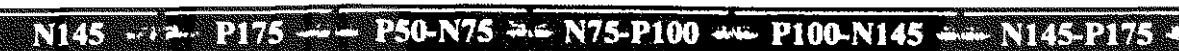 } \\
\hline & & & & & LAT ms & LAT $\mathrm{ms}$ & $A M P u V$ & AMP uV & AMP UV & AMP uV \\
\hline \multirow[t]{6}{*}{ MSLBI } & $1-50$ & 69.8 & 82.7 & 125.5 & 155.5 & 179.5 & 4.7 & 8.5 & 12.7 & 6.7 \\
\hline & $51-100$ & 65.5 & 85.3 & 126.4 & 149.5 & 179.5 & 4.8 & 6.6 & 11.5 & 7.2 \\
\hline & $101-150$ & 60.4 & 84.4 & 129.8 & 159.0 & 188.1 & 9.8 & 15.4 & 16.1 & 6.6 \\
\hline & $151-200$ & 63.0 & 87.0 & 1307 & 160.7 & 186.4 & 12.0 & 18.0 & 18.4 & 0.6 \\
\hline & MEAN & 64.7 & 84.9 & 128.1 & 156.2 & 183.4 & 7.8 & 12.1 & 14.7 & 5.3 \\
\hline & SDT DEV & 4.0 & 1.8 & 2.5 & 4.9 & 4.5 & 3.7 & 5.4 & 3.2 & 3.1 \\
\hline \multirow[t]{6}{*}{ MSRBI } & $1-50$ & 69,0 & 88.7 & 127.3 & 153.0 & 177.0 & 3.9 & 7.1 & 9.5 & 2.2 \\
\hline & $51-100$ & 65.5 & 85.3 & 124.7 & 154.7 & 186.4 & 5.5 & 14.3 & 160 & 3.5 \\
\hline & $101-150$ & 65.5 & 86.1 & 128.1 & 151.3 & 172.7 & 4.1 & 4.5 & 4.2 & 1.7 \\
\hline & $151-200$ & 69.8 & 93.8 & 129.8 & 153.0 & 171.0 & 5.9 & 8.0 & 12.1 & 2.9 \\
\hline & MEAN & 67.5 & 88.5 & 127.5 & 153.0 & 176.8 & 4.9 & 8.5 & 10.5 & 2.6 \\
\hline & SDT DEV & 2.3 & 3.8 & 2.1 & 1.4 & 6.9 & 1.0 & 4.2 & 4.9 & 0.8 \\
\hline \multirow[t]{6}{*}{ MSLGI } & 1.50 & 61.3 & 78.4 & 98.1 & 148.7 & 186.4 & 11.4 & 15.2 & 17.1 & 19.6 \\
\hline & $51-100$ & 63.8 & 78.4 & 96.4 & 147.0 & 192.4 & 8.0 & 114 & 22.2 & 27.7 \\
\hline & $101-150$ & 50.1 & 77.5 & 99.0 & 145.3 & 195.8 & 10.8 & 14.5 & 20.1 & 21.0 \\
\hline & $151-200$ & 51.8 & 80.1 & 99.0 & 146.1 & 190.7 & 79 & 15.0 & 15.6 & 12.4 \\
\hline & MEAN & 56.8 & 78.6 & 98.1 & 146.8 & 191.3 & 9.5 & 14.0 & 18.8 & 20.2 \\
\hline & STD DEV & 6.8 & 1.1 & 1.2 & 1.5 & 3.9 & 1.8 & 1.8 & 3.0 & 6.3 \\
\hline \multirow[t]{6}{*}{ MSRGI } & $1-50$ & 63.0 & 76.7 & 99.0 & 148.7 & 190.7 & 0.6 & 1.0 & 2.1 & 3.8 \\
\hline & $51-100$ & 63,8 & 83.5 & 100.7 & 152.1 & 190.7 & 1.9 & 0.7 & 1.9 & 4.0 \\
\hline & $101-150$ & 63.0 & 86.1 & 105.0 & 145.3 & 184.7 & 1.7 & 9.3 & 2.2 & 14.2 \\
\hline & $151-200$ & 604 & 83.5 & 99.8 & 1513 & 200.1 & 10.1 & 12.1 & 10.7 & 20.2 \\
\hline & MEAN & 62.6 & 82.5 & 101.1 & 149.4 & 191.6 & 3.6 & 5.8 & 4.2 & 10.6 \\
\hline & STD DEV & 1.5 & 4.0 & 2.7 & 3.1 & 6.4 & 4.4 & 5.8 & 4.3 & 8.1 \\
\hline
\end{tabular}

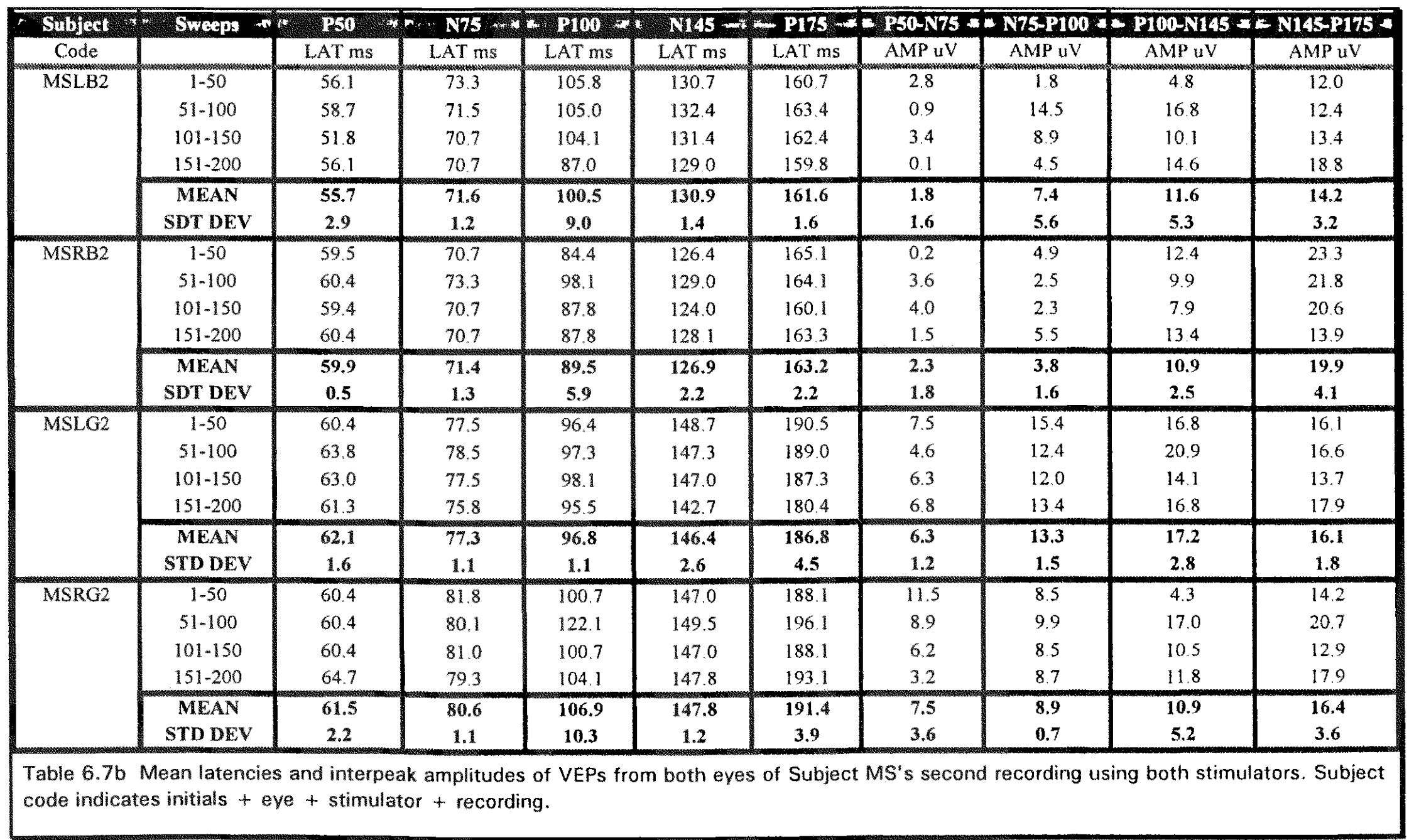




\section{Mean Latencies from first recording - Subject MS}

\section{LED-BOX STIMULATOR}

LED-GOGGLE STIMULATOR

Left Eye Right Eye

Left Eye

Right Eye

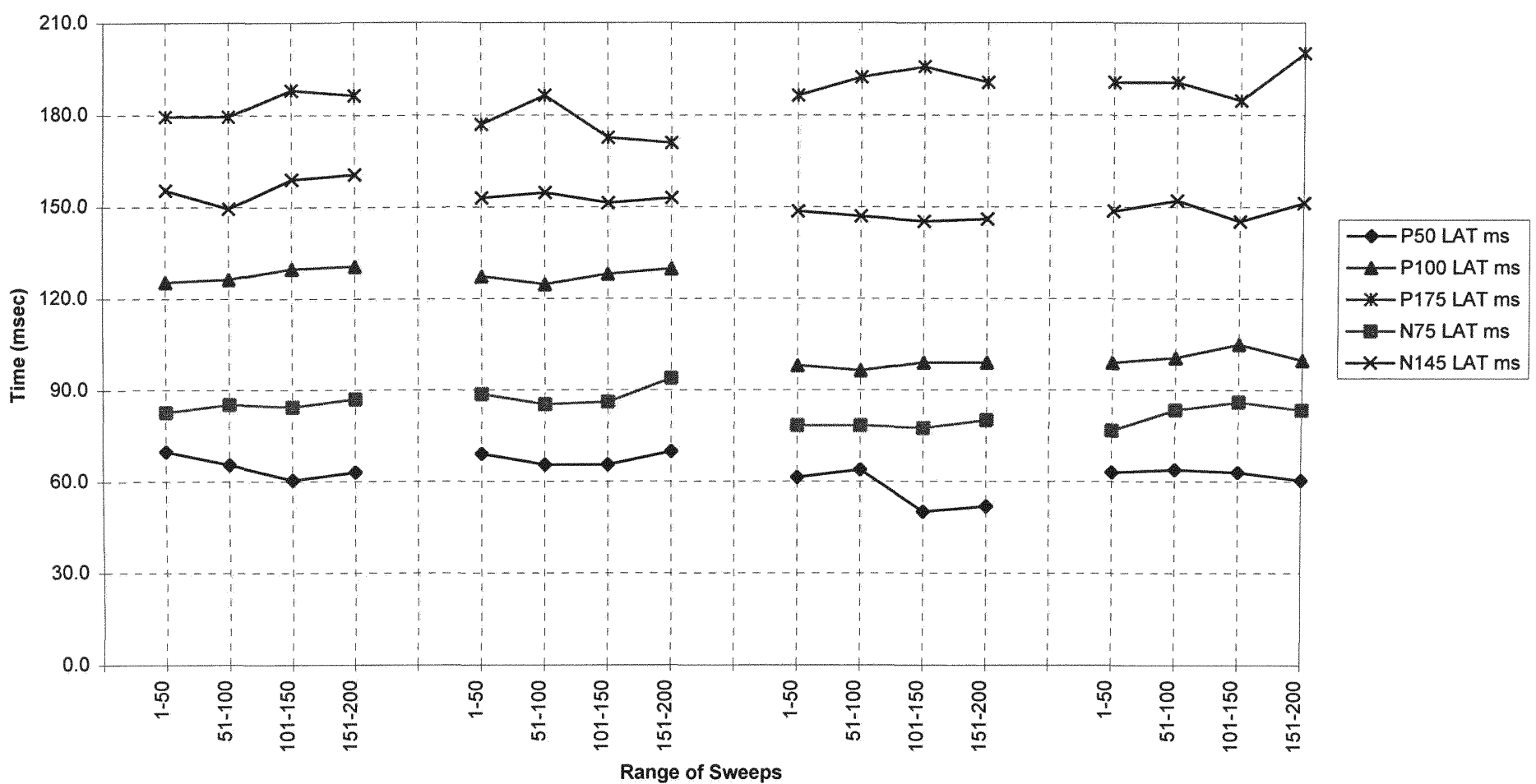

Figure 6.7a Mean latencies in four different ranges of sweeps using both stimulators 


\section{Mean Latencies from second recording - Subject MS}

\section{LED-BOX STIMULATOR}

\section{LED-GOGGLE STIMULATOR}
Left Eye
Right Eye
Left Eye
Right Eye

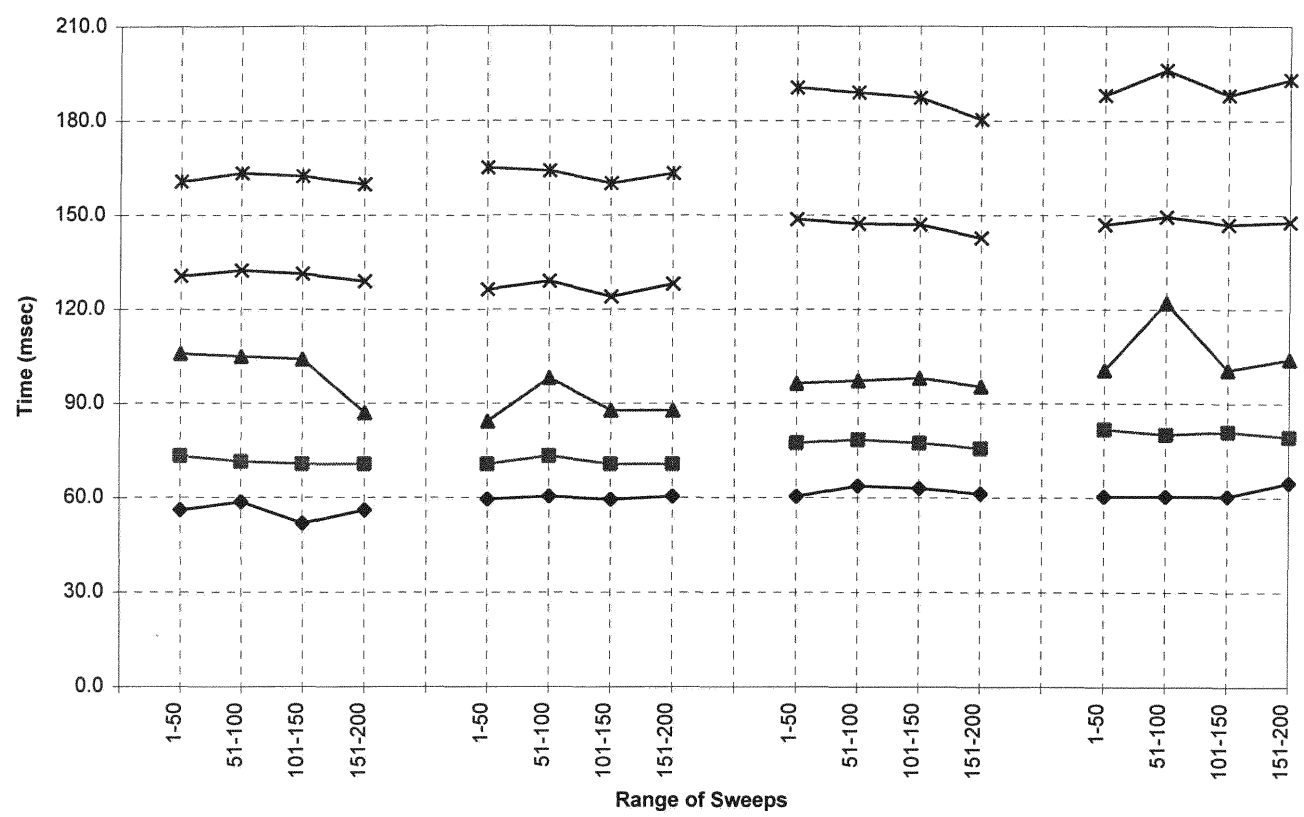

Figure $6.7 \mathrm{~b}$ Mean latencies in four different ranges of sweeps using both stimulators 
Interpeak Amplitudes from first recording - Subject MS

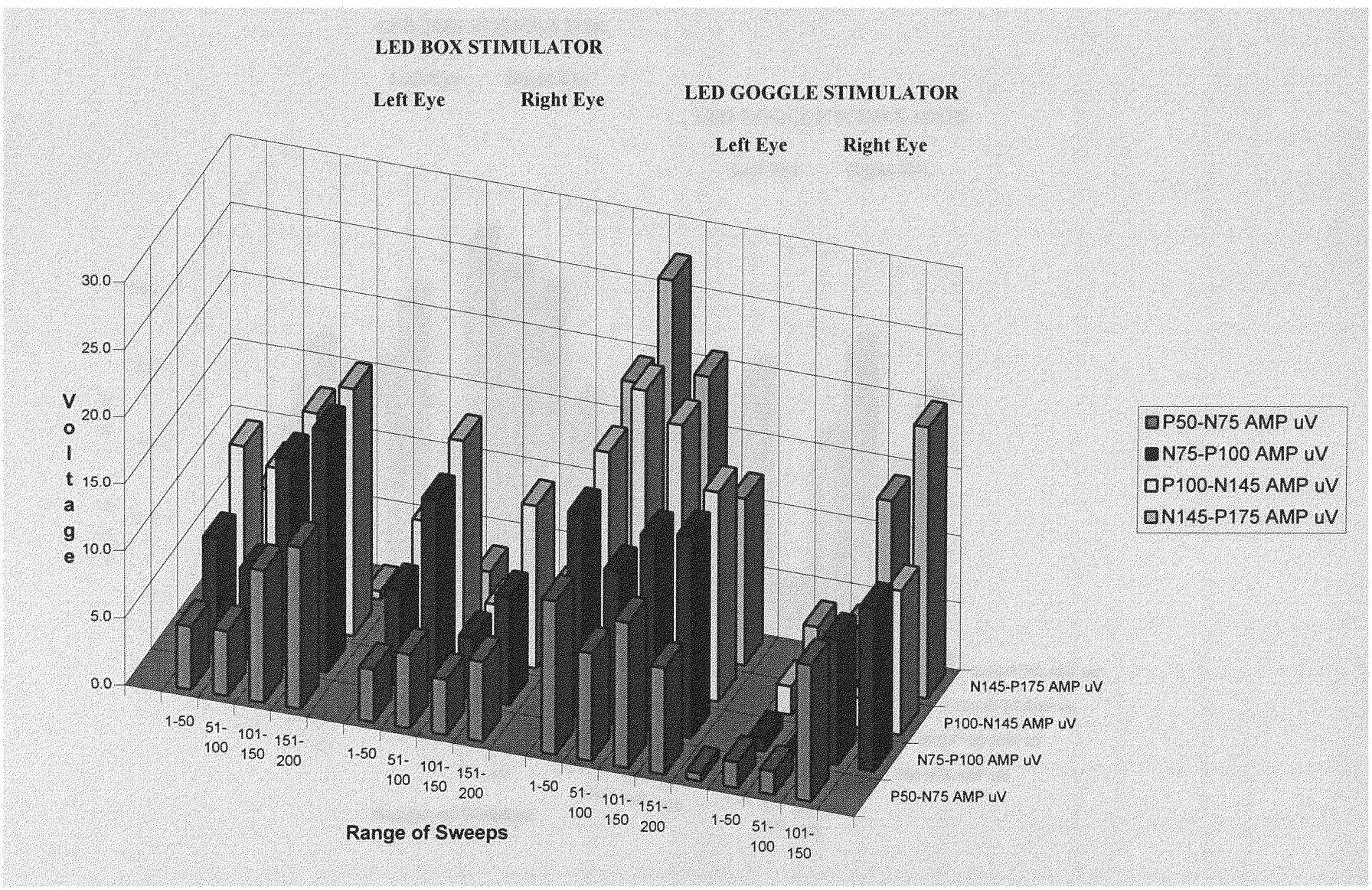

Figure 6.7c Interpeak amplitudes in four different ranges of sweeps using both stimulators 


\section{Interpeak Amplitudes from second recording - Subject MS}

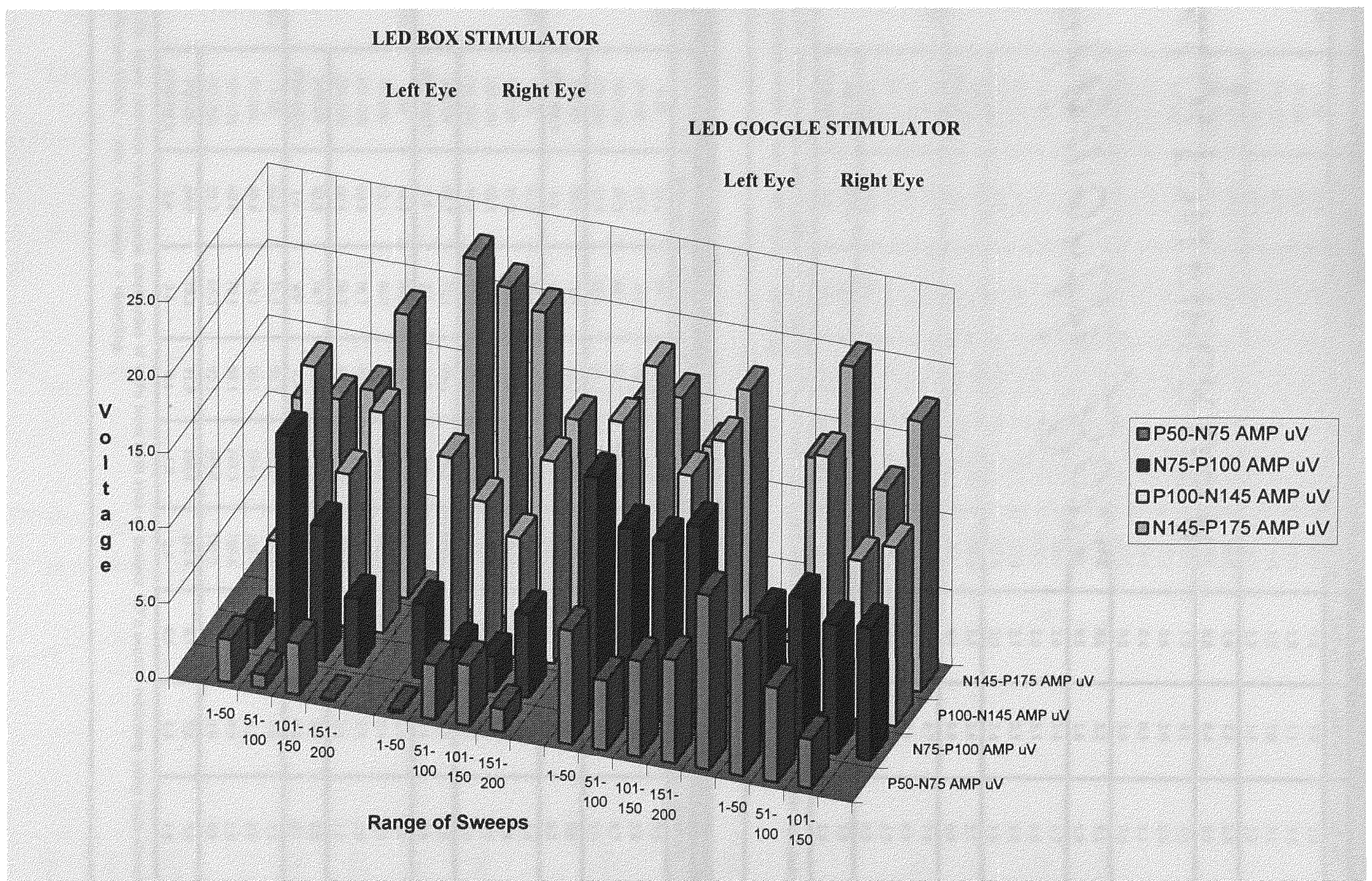

Figure $6.7 \mathrm{~d}$ Interpeak amplitudes in four different ranges of sweeps using both stimulators 


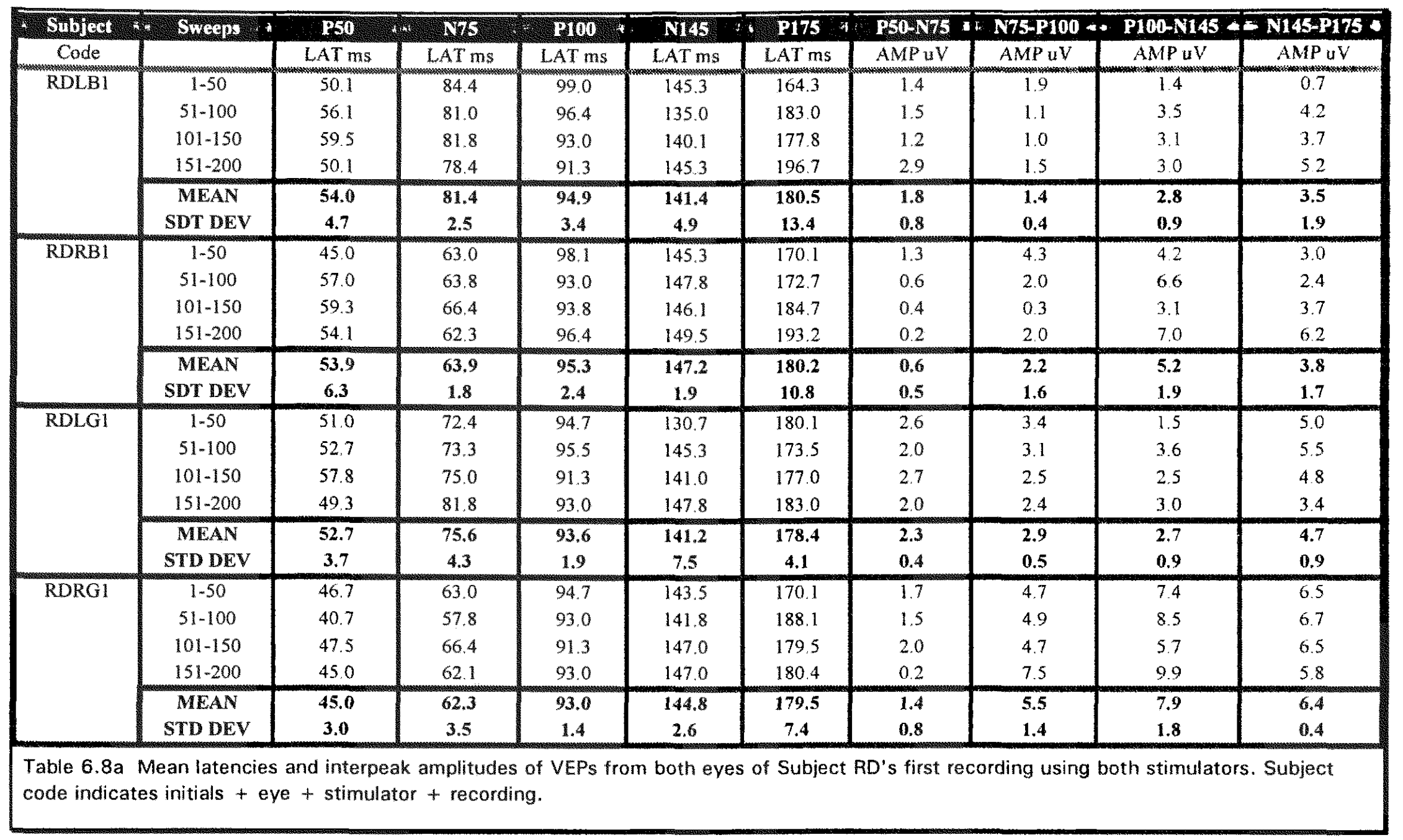

\begin{tabular}{|c|c|c|c|c|c|c|c|c|c|c|}
\hline Subject & Swceps & P30 & N75 & P100 & F115 & P175 & P50N76 1 & N75-P100 & P100N145. & 11752175 \\
\hline Code & & LAT ms & LAT ms & LAT ms & LAT $\mathrm{ms}$ & LAT ms & AMP UV & AMP uV & $\mathrm{AMP}$ uV & AMP uV \\
\hline \multirow[t]{6}{*}{ RDLB2 } & $1-50$ & 57.3 & 68.1 & 96.4 & 147.8 & 193.3 & 0.5 & 1.5 & 5.5 & 6.0 \\
\hline & $51-100$ & 57.0 & 77.5 & 95.5 & 147.8 & 183.0 & 1.8 & 1.8 & 5.8 & 5.3 \\
\hline & $101-150$ & 57.8 & 68.1 & 92.1 & 147.8 & 193.3 & 0.5 & 0.2 & 5.7 & 9.1 \\
\hline & $151-200$ & 55.3 & 66.6 & 91.3 & 148.7 & 194.1 & 1.7 & 3.0 & 5.4 & 4.0 \\
\hline & MEAN & 56.9 & 70.1 & 93.8 & 148.0 & 190.9 & 1.1 & 1.6 & 5.6 & 6.1 \\
\hline & SDT DEV & 1.1 & 5.0 & 2.5 & 0.4 & 5.3 & 0.7 & 1.2 & 0.2 & 2.2 \\
\hline \multirow[t]{6}{*}{ RDRB2 } & $1=50$ & 51.8 & 75.8 & 99.8 & 144.4 & 189.8 & 1.3 & 1.9 & 1.4 & 2.8 \\
\hline & $51-100$ & 57.0 & 75.0 & 96.4 & 139.3 & 189.0 & 1.4 & 1.1 & 2.4 & 4.3 \\
\hline & $101-150$ & 58.7 & 78.4 & 93.0 & 140.1 & 187.3 & 1.1 & 10 & 30 & 3.9 \\
\hline & $151-200$ & 55.3 & 79.3 & 93.0 & 143.1 & 188.1 & 3.0 & 1.7 & 3.0 & 5.3 \\
\hline & MEAN & 55.7 & 77.1 & 95.6 & 141.7 & 188.6 & 1.7 & 1.4 & 2.5 & 4.1 \\
\hline & SDT DEV & 2.9 & 2.1 & & 2.4 & 1.1 & 0.9 & 0.4 & 0.8 & 1.0 \\
\hline \multirow[t]{6}{*}{ RDLG2 } & $1-50$ & 64.7 & 76.7 & 92.1 & 147.0 & 187.3 & 2.1 & 2.4 & 3.2 & 5.2 \\
\hline & $51-100$ & 62.1 & 76.7 & 92.1 & 147.8 & 189.0 & 1.3 & 2.1 & 3.2 & 4.9 \\
\hline & $101-150$ & 63.8 & 76.7 & 92.1 & 147.0 & 188.0 & 1.0 & 2.3 & 30 & 3.5 \\
\hline & $151-200$ & 60.4 & 76.7 & 92.1 & 144.4 & 183.8 & 1.6 & 2.6 & 1.8 & 2.3 \\
\hline & MEAN & 62.8 & 76.7 & 92.1 & 146.6 & 187.0 & 1.5 & 2.4 & 2.8 & 4.0 \\
\hline & STD DEV & 1.9 & 0.0 & 0.0 & 1.5 & 2.3 & 0.5 & 0.2 & 0.7 & 1.3 \\
\hline \multirow[t]{6}{*}{$\mathrm{RDRG} 2$} & $1-50$ & 63.0 & 71.5 & 91.3 & 141.8 & 180.4 & 0.8 & 4.2 & 7.5 & 7.2 \\
\hline & $51-100$ & 60.4 & 74.1 & 92.1 & 1458 & 186.4 & 0.1 & 4.5 & 8.8 & 6.7 \\
\hline & $101-150$ & 60.4 & 70.1 & 92.1 & 145.3 & 183.0 & 1.3 & 4.9 & 5.6 & 7.2 \\
\hline & $151-200$ & 55.7 & 65.5 & 92.1 & 1453 & 1813 & 0.5 & 7.0 & 9.8 & 5.7 \\
\hline & MEAN & 59.9 & 70.3 & 91.9 & 144.6 & 182.8 & 0.7 & 5.2 & 7.9 & 6.7 \\
\hline & STD DEV & 3.0 & 3.6 & 0.4 & 1.8 & 2.6 & 0.5 & 1.3 & 1.8 & 0.7 \\
\hline
\end{tabular}


Mean Latencies from first recording - Subject RD

\section{LED-BOX STIMULATOR}

Left Eye

Right Eye

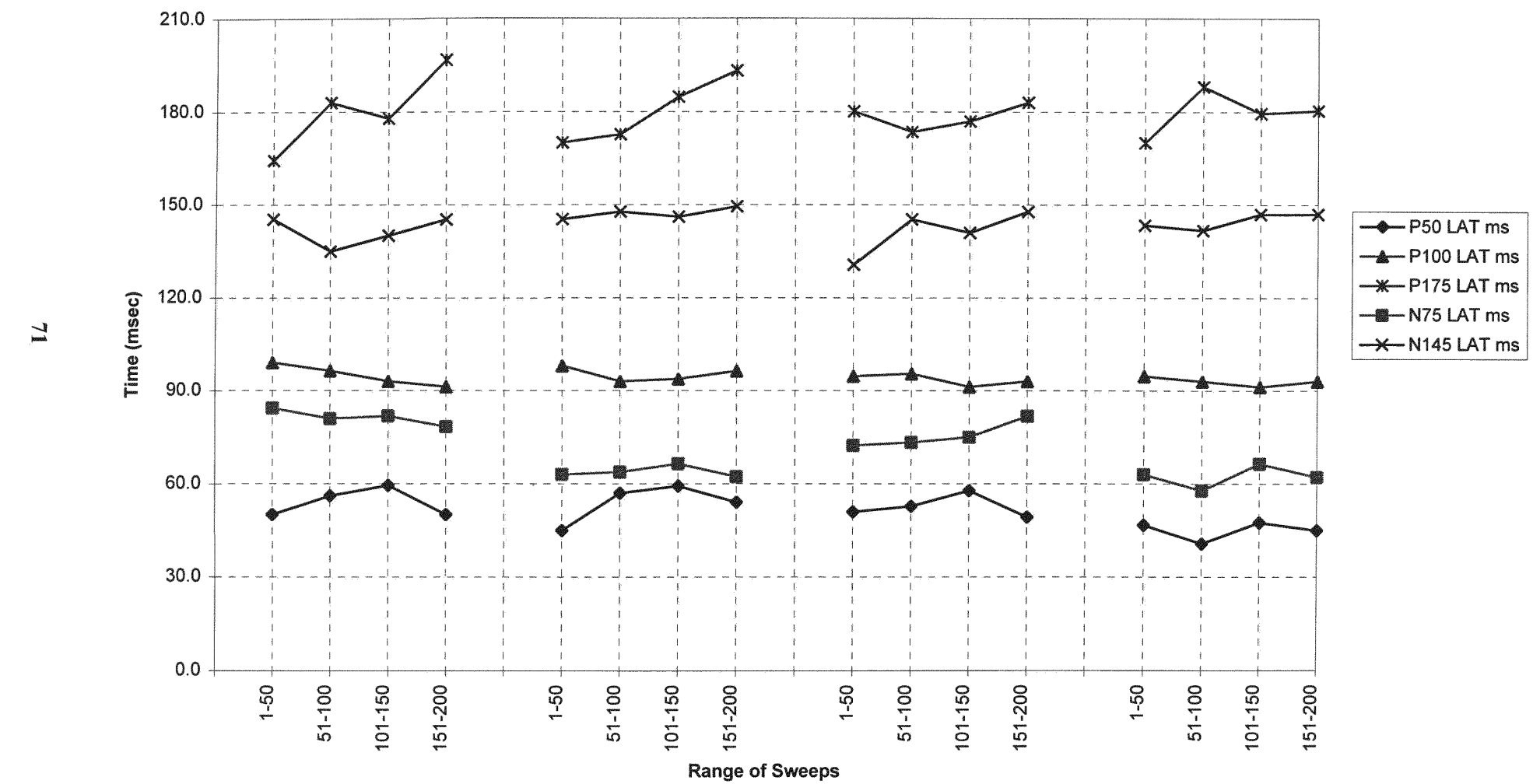

LED-GOGGLE STIMULATOR

Left Eye Right Eye

Figure 6.8a Mean latencies in four different ranges of sweeps using both stimulators 
Mean Latencies from second recording - Subject RD

\section{LED-BOX STIMULATOR \\ LED-GOGGLE STIMULATOR}

$\begin{array}{lll}\text { Left Eye } & \text { Right Eye Left Eye Right Eye }\end{array}$

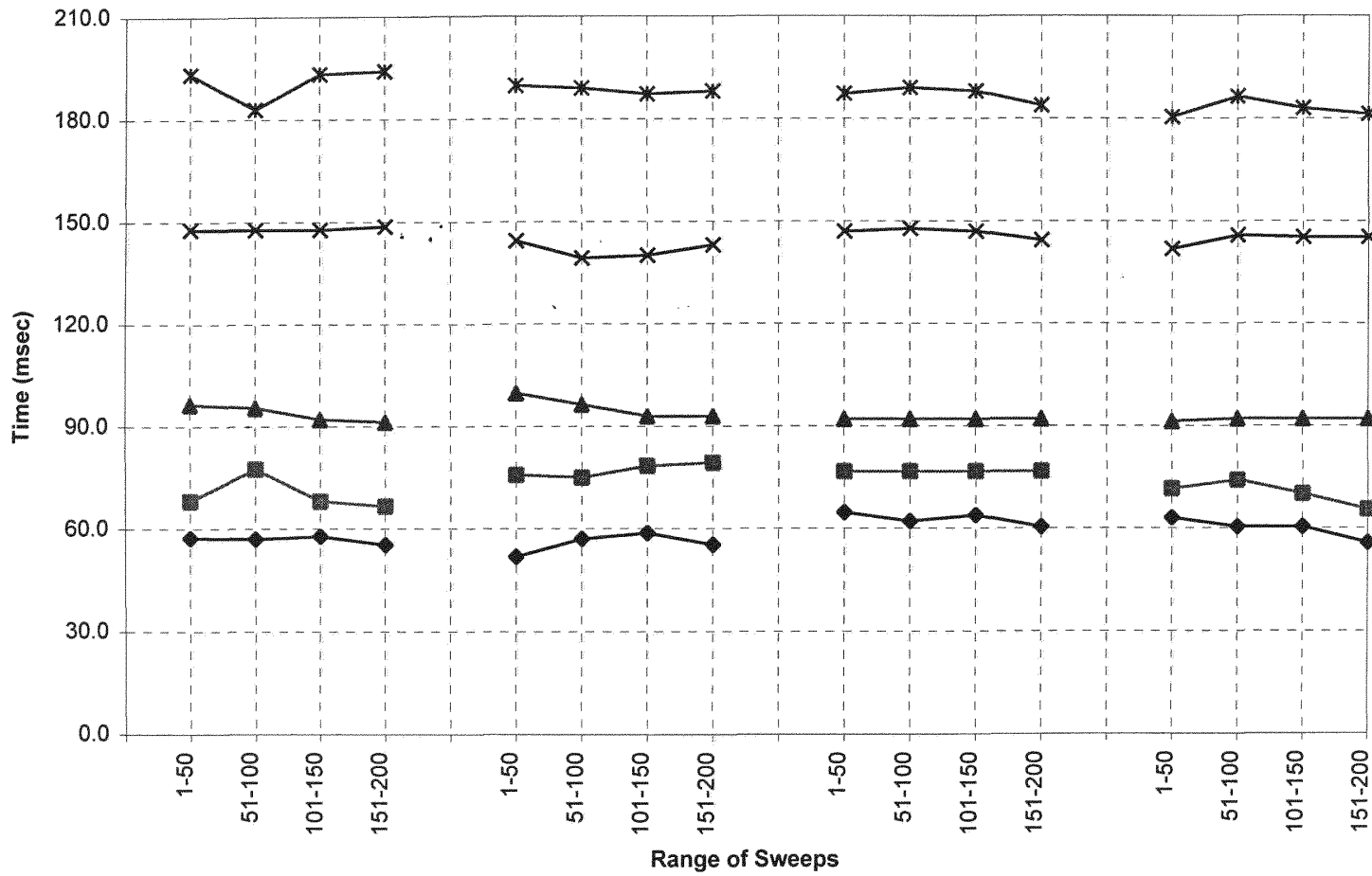

Figure 6.8b Mean latencies in four different ranges of sweeps using both stimulators 


\section{Interpeak Amplitudes from first recording - Subject RD}

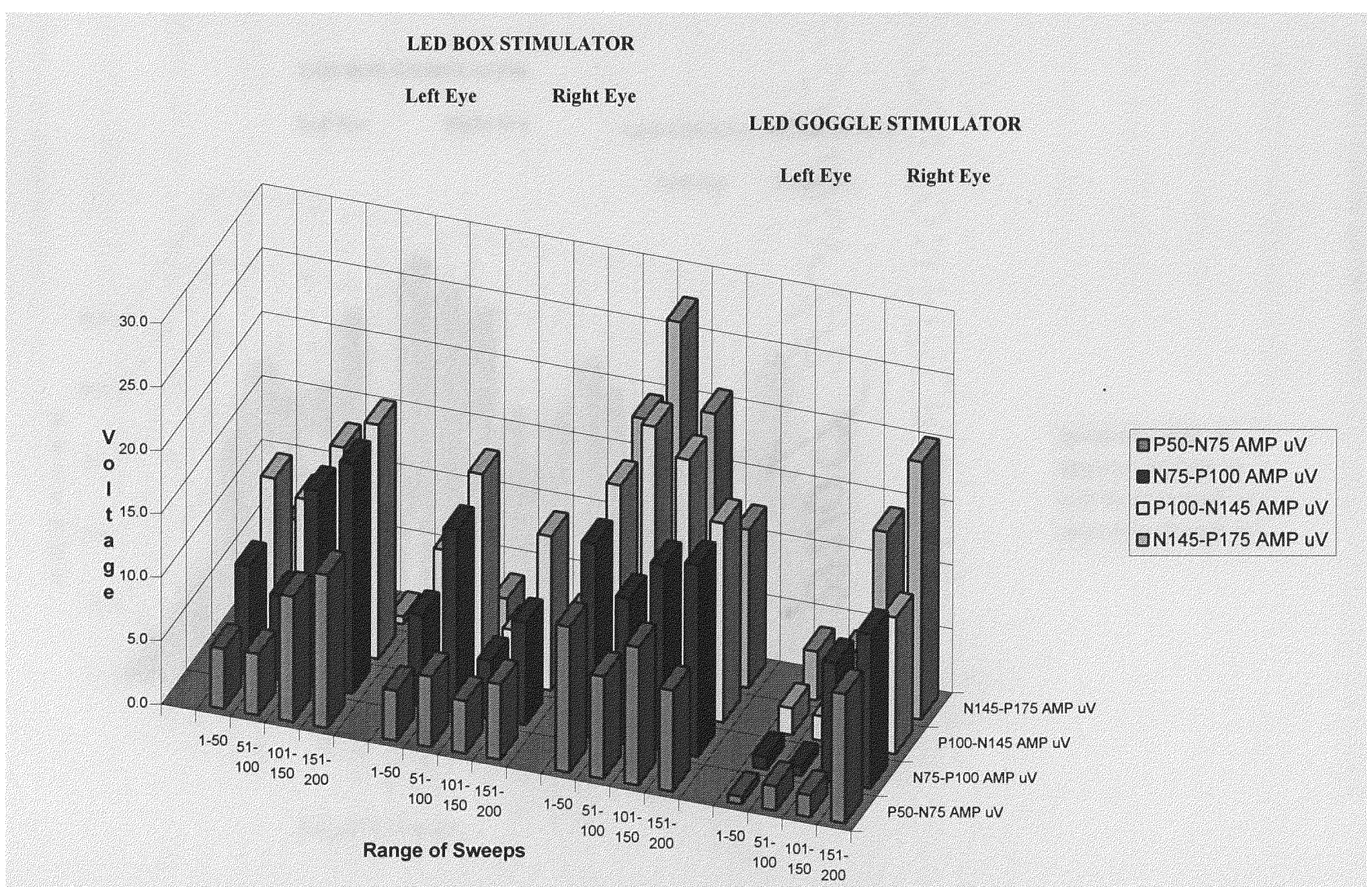

Figure $6.8 \mathrm{c}$ Interpeak amplitudes in four different ranges of sweeps using both stimulators 


\section{Interpeak Amplitudes from second recording - Subject RD}

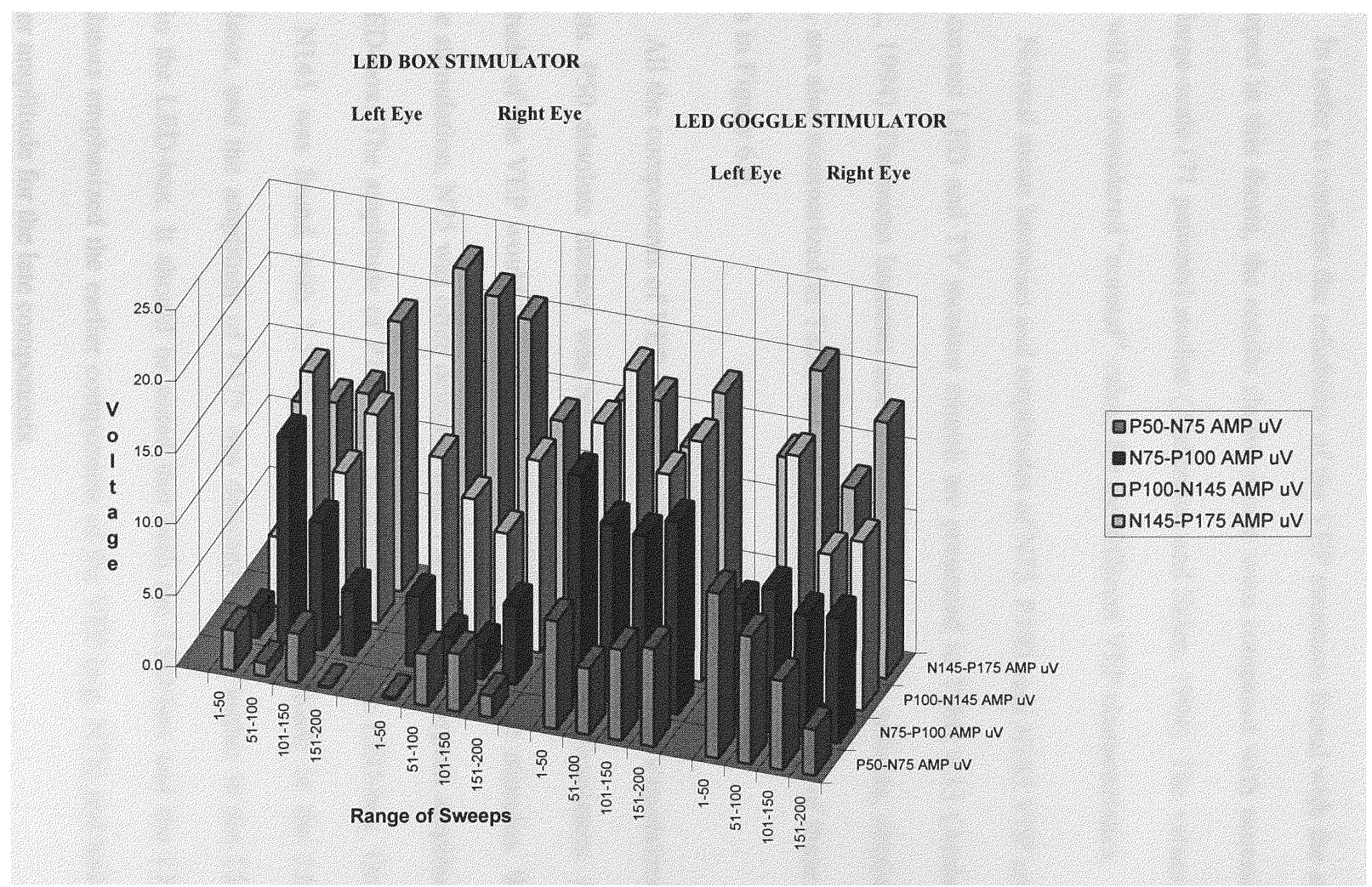

Figure 6.8d Interpeak amplitudes in four different ranges of sweeps using both stimulators 


\subsection{Observations on the results and comparison with other data}

In order to confirm the reliability of the VEP measures found with the stimulators developed in this thesis, the results shown above were compared with measures obtain from large-scale (71 patients) studies (Andersson and Siden, 1994). The values from that study will be considered "normal" values for the different VEP characteristics.

Normal mean latencies and amplitudes of N75, P100, and N145 VEP components for a current LED and TV monitor stimuli are presented in Table 6.13 (Andersson and Siden., 1994). The mean latencies and amplitudes from all the patients, obtained in this thesis, are also summarized in Table 6.9 and graphical representations of these tables are shown in Figure 6.9.

All the components of VEP recording were found with both stimulators in all the subjects. P50 absolute latency was relatively the same in both stimulators. A reduced amplitude of the VEP would be expected with the low-luminance stimulus. With LEDgoggle stimulation, N75 was often detected with $1 \mathrm{uV}$ higher interpeak amplitude than in the LED-box. The amplitude of P100 was $0.2 \mathrm{uV}$ higher in the LED-box. On the other hand, N145 was found with $1.5 \mathrm{uV}$ higher interpeak amplitude in the LED-goggle stimulator, and the amplitude of P175 was detected slightly higher in the LED-goggle than in the LED-box. It should be noted that both the LED-box and the LED-goggle stimulators emphasized the earlier components of the VEP (e.g. N75) and had a slightly smaller amplitude for the late components. 
The N75 mean amplitude of the LED-goggle was $4.8 \mathrm{uV}$ higher than the normal mean amplitude of the Andersson's LED-box. Furthermore, P100 mean amplitude was also $0.6 \mathrm{uV}$ higher than the Andersson's LED-box stimulator. However, N145 mean amplitude was $3.8 \mathrm{uV}$ lower than the normal mean amplitude found in the Andersson's LED-box.

With LED-goggle, N75, P100 and N145 were found 1.2, 5.6 and 1.0 milliseconds shorter absolute latency with respect to the LED-box absolute latencies. Furthermore, P175 was also found shorter latency in the LED-goggle rather than in the LED-box. On the other hand, the latencies for all peaks obtained with both the LED-box and LED-goggle stimulators always fall between the latencies found with Andersson's LED-box and TV monitor stimulators. Furthermore, the most clinically significant peak of the VEP, i.e., P100, (Spehlmann, 1985) had about the same latency for all stimulators. 


\begin{tabular}{|l|c|c|c|c|}
\hline Latencies & $\begin{array}{c}\text { Normal Mean } \\
\text { Latency } \\
(\mathrm{msec})\end{array}$ & $\begin{array}{c}\text { Standard } \\
\text { Deviation }\end{array}$ & $\begin{array}{c}\text { Normal Mean } \\
\text { Amplitude } \\
(\mathrm{uV})\end{array}$ & $\begin{array}{c}\text { Normal } \\
\text { Range }\end{array}$ \\
\hline N75 LED & 88.3 & 6.9 & 2.0 & $0-7.0$ \\
N75 TV & 72.8 & 3.4 & 3.3 & $0-10.0$ \\
P100 LED & 118.4 & 5.3 & 7.4 & $1.4-19.6$ \\
P100 TV & 97.4 & 5.8 & 7.6 & $2.1-19.0$ \\
N145 LED & 155.2 & 5.8 & 12.0 & $4.1-27.0$ \\
N145 TV & 131.2 & 11.6 & 8.4 & $1.2-25.0$ \\
\hline
\end{tabular}

\begin{tabular}{|c|c|c|c|c|}
\hline Latencies & $\begin{array}{c}\text { Mean Latency } \\
(\mathrm{msec})\end{array}$ & $\begin{array}{c}\text { Standard } \\
\text { Deviation }\end{array}$ & $\begin{array}{c}\text { Mean } \\
\text { Amplitude } \\
(\mathrm{uV})\end{array}$ & Range \\
\hline N75 BOX & 77.5 & 6.2 & 5.9 & $1.4-12.2$ \\
N75 GOGGLE & 76.3 & 6.0 & 6.8 & $1.4-14.0$ \\
P100 BOX & 111.4 & 11.6 & 8.2 & $2.8-18$ \\
P100 GOGGLE & 105.9 & 10.8 & 8.0 & $1.4-18.8$ \\
N145 BOX & 143.9 & 6.8 & 6.7 & $0.8-20$ \\
N145 GOGGLE & 142.8 & 7.8 & 8.2 & $1.0-20.2$ \\
\hline
\end{tabular}

Table 6.9 Top table shows the normal mean latencies and mean amplitudes of N75, P100 and N145 from 71 subjects' right eye ( 32 males and 39 females) performed by Andersson et al with a TV monitor and LED-Box stimulators. Bottom table shows the mean latencies and mean amplitude from this study using LED-Box and LED-Goggle. 


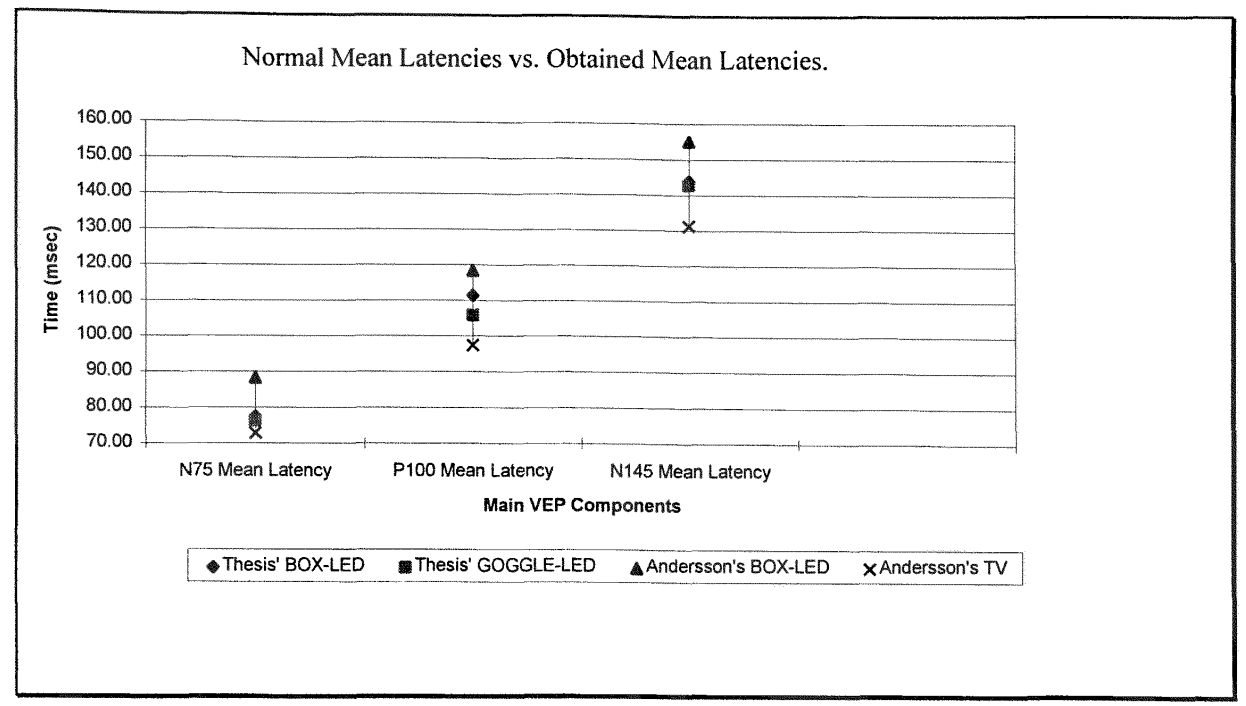

Figure 6.9a Comparison of normal mean latencies versus thesis' LED-Box and LEDGoggle mean latencies.

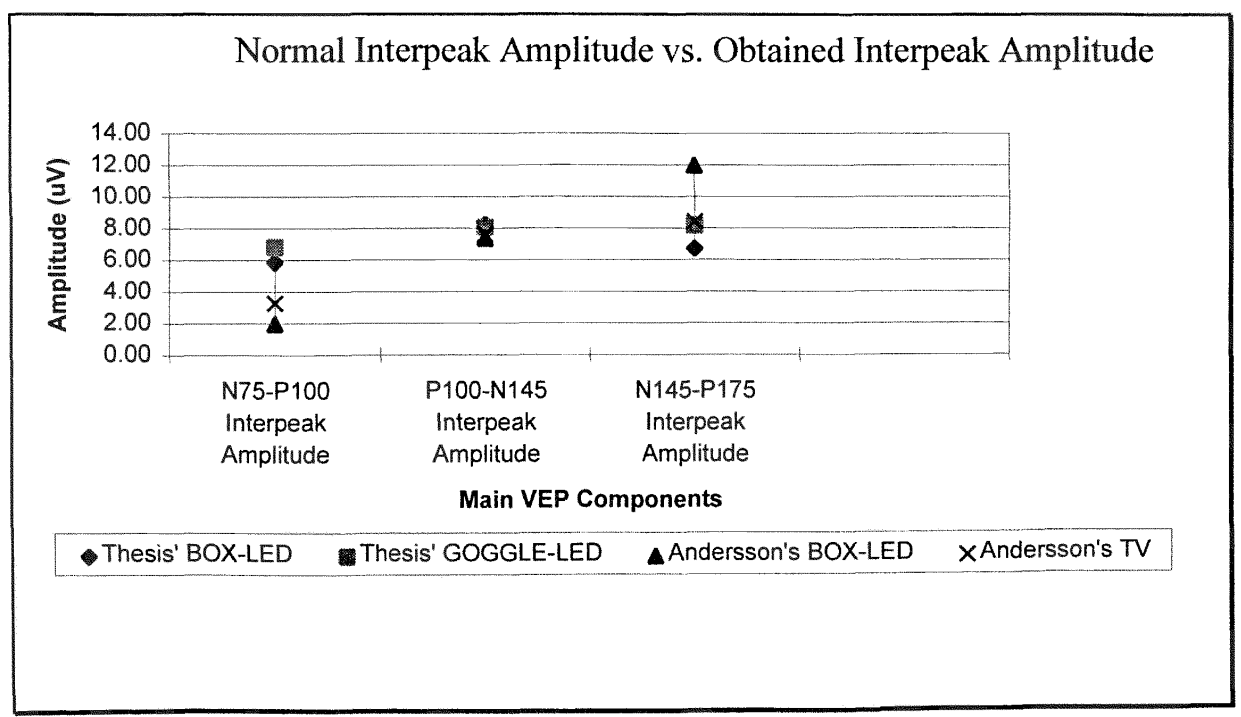

Figure 6.9b Comparison of normal interpeak amplitudes versus thesis' LED-Box and LED-Goggle interpeak amplitudes. 


\subsection{Interpeak Latencies}

Latencies measured between peaks help to localize lesions to the structure in the central sensory path assumed to generate these peaks. Interpeak latencies vary less than peak latencies and are more sensitive to pathology; they are therefore better indicator of abnormal functions (Spehlmann, 1985). Differences of interpeak latencies between VEP peaks recorded from midline in response to successive stimulations of the eye on either side (interocular latency difference) or between VEP recorded simultaneously on both sides of the head (left-right latency difference) may suggest an abnormality of the VEP having longer latency.

Interpeak latencies of the most studied peaks such as: P50-N75, N75-P100, and P100-N145, N145-P175 obtained with both stimulators are shown in Table $6.10-6.15$. Furthermore, these interpeak latencies are also compared with the Andersson's LED-box stimulator and TV monitor. 


\begin{tabular}{|c|c|c|c|c|c|}
\hline Subject & Sweeps & P50-N75 & N75-P100 & P100-N145 & N145-P175 \\
\hline Code & & LAT msec & LAT msec & LAT msec & LAT msec \\
\hline \multirow[t]{6}{*}{ CLLBI } & $1-50$ & 28.3 & 36.8 & 29.2 & 39.4 \\
\hline & $51-100$ & 27.3 & 40.4 & 26.6 & 36.8 \\
\hline & $101-150$ & 38.6 & 31.7 & 25.7 & 37.7 \\
\hline & $151-200$ & 40.3 & 36.0 & 29.1 & 29.2 \\
\hline & MEAN & 33.6 & 36.2 & 27.7 & 35.8 \\
\hline & SDT DEV & 6.8 & 3.6 & 1.8 & 4.5 \\
\hline \multirow[t]{6}{*}{ CLRB1 } & $1-50$ & 23.2 & 44.6 & 23.1 & 44.9 \\
\hline & $51-100$ & 24.8 & 42.9 & 26.6 & 41.1 \\
\hline & $101-150$ & 28.3 & 38.6 & 24.0 & 38.6 \\
\hline & $151-200$ & 28.3 & 26.5 & 24.9 & 37.7 \\
\hline & MEAN & 26.2 & 38.2 & 24.7 & 40.6 \\
\hline & SDT DEV & 2.6 & 8.2 & 1.5 & 3.2 \\
\hline \multirow[t]{6}{*}{ CLLG1 } & $1-50$ & 36.0 & 24.0 & 30.8 & 31.7 \\
\hline & $51-100$ & 28.3 & 17.2 & 23.1 & 19.7 \\
\hline & $101-150$ & 12.0 & 21.4 & 27.5 & 40.2 \\
\hline & $151-200$ & 26.4 & 19.7 & 30.0 & 43.7 \\
\hline & MEAN & 25.7 & 20.6 & 27.9 & 33.8 \\
\hline & STD DEV & 10.0 & 2.9 & 3.5 & 10.7 \\
\hline \multirow[t]{6}{*}{ CLRG1 } & $1-50$ & 28.3 & 29.2 & 39.4 & 39.4 \\
\hline & $51-100$ & 29.5 & 37.4 & 25.7 & 42.0 \\
\hline & $101-150$ & 25.7 & 42.0 & 27.4 & 20.6 \\
\hline & $151-200$ & 30.9 & 38.6 & 36.0 & 42.8 \\
\hline & MEAN & 28.6 & 36.8 & 32.1 & 36.2 \\
\hline & STD DEV & 2.2 & 5.4 & 6.6 & 10.5 \\
\hline
\end{tabular}

Table 6.10 a Interpeak latencies from the main components of SUBJECT CL first VEP response using both stimulators. 


\begin{tabular}{|c|c|c|c|c|c|}
\hline Subject & Sweeps & P50-N75 & N75-P100 & P100-N145 & N145-P175 \\
\hline Code & & LAT msec & IAT msec & LAT msec & LAT msec \\
\hline \multirow[t]{6}{*}{ CLLB2 } & $1-50$ & 28.2 & 38.7 & 24.9 & 35.1 \\
\hline & $51-100$ & 24.0 & 44.6 & 24.9 & 35.1 \\
\hline & $101-150$ & 20.6 & 56.5 & 29.2 & 26.6 \\
\hline & $151-200$ & 22.3 & 45.4 & 37.7 & 30.9 \\
\hline & MEAN & 23.8 & 46.3 & 29.2 & 31.9 \\
\hline & SDT DEV & 3.3 & 7.4 & 6.0 & 4.1 \\
\hline \multirow[t]{6}{*}{ CLRB2 } & $1-50$ & 32.5 & 27.5 & 34.3 & 35.1 \\
\hline & $51-100$ & 24.8 & 41.5 & 29.7 & 36.8 \\
\hline & $101-150$ & 18.4 & 45.4 & 29.2 & 40.2 \\
\hline & $151-200$ & 15.0 & 42.6 & 28.3 & 36.0 \\
\hline & MEAN & 22.7 & 39.3 & 30.4 & 37.0 \\
\hline & SDT DEV & 7.7 & 8.0 & 2.7 & 2.2 \\
\hline \multirow[t]{6}{*}{ CLLG2 } & $1-50$ & 9.5 & 36.8 & 31.4 & 39.9 \\
\hline & $51-100$ & 13.7 & 27.4 & 35.2 & 35.0 \\
\hline & $101-150$ & 12.4 & 27.9 & 40.3 & 36.0 \\
\hline & $151-200$ & 26.4 & 19.7 & 33.5 & 40.2 \\
\hline & MEAN & 15.5 & 28.0 & 35.1 & 37.8 \\
\hline & STD DEV & 7.5 & 7.0 & 3.8 & 2.7 \\
\hline \multirow[t]{6}{*}{ CLRG2 } & $1-50$ & 18.7 & 31.9 & 34.3 & 44.5 \\
\hline & $51-100$ & 25.0 & 30.9 & 27.4 & 42.9 \\
\hline & $101-150$ & 25.7 & 33.4 & 30.0 & 45.5 \\
\hline & $151-200$ & 16.3 & 36.0 & 30.9 & 48.8 \\
\hline & MEAN & 21.4 & 33.1 & 30.7 & 45.4 \\
\hline & STD DEV & 4.6 & 2.2 & 2.9 & 2.5 \\
\hline
\end{tabular}

Table $6.10 \mathrm{~b}$ Interpeak latencies from the main components of SUBJECT CL first VEP response using both stimulators. 


\begin{tabular}{|c|c|c|c|c|c|}
\hline Subject & Sweeps & P50-N75 & N75-P100 & P100-N145 & N145-P175 \\
\hline Code & & LAT msec & LAT msec & LAT msec & LAT msec \\
\hline \multirow[t]{6}{*}{ CQLBI } & $1-50$ & 19.7 & 25.8 & 38.5 & 44.6 \\
\hline & $51-100$ & 6.9 & 33.4 & 31.7 & 48.9 \\
\hline & $101-150$ & 13.7 & 23.2 & 39.4 & 43.7 \\
\hline & $151-200$ & 14.6 & 32.7 & 29.9 & 52.3 \\
\hline & MEAN & 13.7 & 28.8 & 34.9 & 47.4 \\
\hline & SDT DEV & 5.3 & 5.1 & 4.8 & 4.0 \\
\hline \multirow[t]{6}{*}{ CQRBI } & $1-50$ & 20.6 & 25.6 & 37.0 & 43.0 \\
\hline & $51-100$ & 6.9 & 34.3 & 31.7 & 48.0 \\
\hline & $101-150$ & 13.7 & 23.2 & 39.4 & 44.6 \\
\hline & $151-200$ & 12.9 & 33.4 & 30.0 & 50.6 \\
\hline & MEAN & 13.5 & 29.1 & 34.5 & 46.6 \\
\hline & SDT DEV & 5.6 & 5.6 & 4.4 & 3.4 \\
\hline \multirow[t]{6}{*}{ CQLG1 } & $1-50$ & 13.2 & 24.0 & 43.7 & 21.0 \\
\hline & $51-100$ & 7.7 & 40.3 & 37.7 & 24.0 \\
\hline & $101-150$ & 24.9 & 30.0 & 46.3 & 27.4 \\
\hline & $151-200$ & 11.7 & 37.1 & 27.4 & 23.2 \\
\hline & MEAN & 14.4 & 32.9 & 38.8 & 23.9 \\
\hline & STD DEV & 7.4 & 7.3 & 8.4 & 2.7 \\
\hline \multirow[t]{6}{*}{ CQRGI } & $1-50$ & 24.6 & 49.1 & 32.6 & 43.7 \\
\hline & $51-100$ & 12.0 & 48.7 & 31.9 & 54.8 \\
\hline & $101-150$ & 17.2 & 48.8 & 44.6 & 27.4 \\
\hline & $151-200$ & 12.6 & 41.4 & 59.1 & 31.6 \\
\hline & MEAN & 16.6 & 47.0 & 42.1 & 39.4 \\
\hline & STD DEV & 5.8 & 3.7 & 12.8 & 12.4 \\
\hline
\end{tabular}

Table 6.11a Interpeak latencies from the main components of SUBJECT CQ first VEP response using both stimulators. 


\begin{tabular}{|c|c|c|c|c|c|}
\hline Subject & Sweeps & P50-N75 & N75-P100 & P100-N145 & N145-P175 \\
\hline Code & & LAT msec & LAT msec & LAT msec & LAT msec \\
\hline \multirow[t]{6}{*}{ CQLB2 } & $1-50$ & 16.3 & 20.6 & 39.4 & 37.7 \\
\hline & $51-100$ & 12.9 & 27.4 & 39.4 & 42.9 \\
\hline & $101-150$ & 15.4 & 24.0 & 37.7 & 40.3 \\
\hline & $151-200$ & 12.0 & 28.3 & 38.6 & 46.3 \\
\hline & MEAN & 14.2 & 25.1 & 38.8 & 41.8 \\
\hline & SDT DEV & 2.0 & 3.5 & 0.8 & 3.7 \\
\hline \multirow[t]{6}{*}{ CQRB2 } & $1-50$ & 8.6 & 32.6 & 36.0 & 36.8 \\
\hline & $51-100$ & 12.8 & 31.7 & 39.5 & 30.0 \\
\hline & $101-150$ & 12.0 & 36.0 & 34.3 & 38.6 \\
\hline & $151-200$ & 12.8 & 36.0 & 43.8 & 33.4 \\
\hline & MEAN & 11.6 & 34.1 & 38.4 & 34.7 \\
\hline & SDT DEV & 2.0 & 2.3 & 4.2 & 3.8 \\
\hline \multirow[t]{6}{*}{ CQLG2 } & $1-50$ & 25.7 & 24.9 & 20.6 & 40.3 \\
\hline & $51-100$ & 22.3 & 23.1 & 36.7 & 31.9 \\
\hline & $101-150$ & 17.2 & 28.2 & 38.6 & 26.6 \\
\hline & $151-200$ & 22.3 & 18.9 & 41.1 & 32.6 \\
\hline & MEAN & 21.9 & 23.8 & 34.3 & 32.9 \\
\hline & STD DEV & 3.5 & 3.9 & 9.3 & 5.6 \\
\hline \multirow[t]{6}{*}{ CQRG2 } & $1-50$ & 10.4 & 34.3 & 43.7 & 26.6 \\
\hline & $51-100$ & 17.2 & 31.7 & 42.0 & 26.6 \\
\hline & $101-150$ & 14.7 & 47.9 & 35.1 & 23.2 \\
\hline & $151-200$ & 15.1 & 48.3 & 36.0 & 23.1 \\
\hline & MEAN & 14.4 & 40.6 & 39.2 & 24.9 \\
\hline & STD DEV & 2.9 & 8.8 & 4.3 & 2.0 \\
\hline
\end{tabular}

Table $6.11 \mathrm{~b}$ Interpeak latencies from the main components of SUBJECT CQ first VEP response using both stimulators. 


\begin{tabular}{|c|c|c|c|c|c|}
\hline Subject & Sweeps & P50-N75 & N75-P100 & P100-N145 & N145-P175 \\
\hline Code & & I.AT msec & LAT msec & LAT msec & LAT msec \\
\hline \multirow[t]{6}{*}{ IMLBI } & $1-50$ & 11.1 & 41.2 & 24.8 & 43.7 \\
\hline & $51-100$ & 8.6 & 42.8 & 20.6 & 43.1 \\
\hline & $101-150$ & 12.8 & 38.6 & 23.2 & 49.7 \\
\hline & $151-200$ & 12.9 & 40.9 & 22.5 & 48.9 \\
\hline & MEAN & 11.4 & 40.9 & 22.8 & 46.4 \\
\hline & SDT DEV & 2.0 & 1.7 & 1.7 & 3.4 \\
\hline \multirow[t]{6}{*}{ IMRBI } & $1-50$ & 12.0 & 41.1 & 24.0 & 52.3 \\
\hline & $51-100$ & 11.1 & 40.3 & 26.6 & 52.3 \\
\hline & $101-150$ & 12.9 & 43.7 & 18.9 & 52.3 \\
\hline & $151-200$ & 9.1 & 39.7 & 32.6 & 48.0 \\
\hline & MEAN & 11.3 & 41.2 & 25.5 & 51.2 \\
\hline & SDT DEV & 1.6 & 1.8 & 5.7 & 2.2 \\
\hline \multirow[t]{6}{*}{ IMLGI } & $1-50$ & 11.1 & 43.7 & 24.0 & 46.6 \\
\hline & $51-100$ & 9.5 & 46.3 & 20.5 & 48.0 \\
\hline & $101-150$ & 9.5 & 43.7 & 21.4 & 49.7 \\
\hline & $151-200$ & 10.3 & 41.1 & 24.0 & 53.2 \\
\hline & MEAN & 10.1 & 43.7 & 22.5 & 49.4 \\
\hline & STD DEV & 0.8 & 2.1 & 1.8 & 2.8 \\
\hline \multirow[t]{6}{*}{ IMRGI } & $1-50$ & 11.2 & 45.4 & 20.6 & 42.8 \\
\hline & $51-100$ & 10.3 & 43.0 & 20.4 & 50.6 \\
\hline & $101-150$ & 11.2 & 46.3 & 18.2 & 46.0 \\
\hline & $151-200$ & 9.5 & 44.5 & 19.7 & 50.9 \\
\hline & MEAN & 10.6 & 44.8 & 19.7 & 47.6 \\
\hline & STD DEV & 0.8 & 1.4 & 1.1 & 3.9 \\
\hline
\end{tabular}

Table $6.12 \mathrm{a}$ Interpeak latencies from the main components of SUBJECT IM first VEP response using both stimulators. 


\begin{tabular}{|c|c|c|c|c|c|}
\hline Subject & Sweeps & P50-N75 & N75-P100 & P100-N145 & N145-P175 \\
\hline Code & & LAT msec & LAT msec & LAT msec & LAT msec \\
\hline \multirow[t]{6}{*}{ IMLB2 } & $1-50$ & 14.6 & 44.5 & 24.9 & 33.4 \\
\hline & $51-100$ & 12.0 & 39.4 & 27.4 & 41.2 \\
\hline & $101-150$ & 12.0 & 35.2 & 26.5 & 44.6 \\
\hline & $151-200$ & 11.2 & 39.3 & 26.7 & 43.7 \\
\hline & MEAN & 12.5 & 39.6 & 26.4 & 40.7 \\
\hline & SDT DEV & 1.5 & 3.8 & 1.1 & 5.1 \\
\hline \multirow[t]{6}{*}{ IMRB2 } & $1-50$ & 10.3 & 42.9 & 24.0 & 29.1 \\
\hline & $51-100$ & 12.9 & 36.0 & 27.4 & 40.3 \\
\hline & $101-150$ & 7.8 & 44.5 & 23.2 & 37.7 \\
\hline & $151-200$ & 8.6 & 47.1 & 20.6 & 43.7 \\
\hline & MEAN & 9.9 & 42.6 & 23.8 & 37.7 \\
\hline & SDT DEV & 2.3 & 4.7 & 2.8 & 6.2 \\
\hline \multirow[t]{6}{*}{ IMLG2 } & $1-50$ & 11.2 & 43.7 & 23.1 & 48.0 \\
\hline & $51-100$ & 8.6 & 45.4 & 21.5 & 48.8 \\
\hline & $101-150$ & 8.6 & 46.3 & 20.6 & 48.0 \\
\hline & $151-200$ & 8.6 & 46.3 & 19.7 & 47.2 \\
\hline & MEAN & 9.3 & 45.4 & 21.2 & 48.0 \\
\hline & STD DEV & 1.3 & 1.2 & 1.5 & 0.7 \\
\hline \multirow[t]{6}{*}{ IMRG2 } & $1-50$ & 11.1 & 42.9 & 21.4 & 46.3 \\
\hline & $51-100$ & 12.0 & 44.5 & 18.9 & 48.0 \\
\hline & $101-150$ & 12.0 & 44.5 & 18.0 & 51.5 \\
\hline & $151-200$ & 9.5 & 44.5 & 20.6 & 49.7 \\
\hline & MEAN & 11.2 & 44.1 & 19.7 & 48.9 \\
\hline & STD DEV & 1.2 & 0.8 & 1.6 & 2.2 \\
\hline
\end{tabular}

Table $6.12 \mathrm{~b}$ Interpeak latencies from the main components of SUBJECT IM first VEP response using both stimulators. 


\begin{tabular}{|c|c|c|c|c|c|}
\hline Subject & Sweeps & P50-N75 & N75-P100 & P100-N145 & N145-P175 \\
\hline Code & & LAT msec & LAT msec & IAT msec & LAT msec \\
\hline \multirow[t]{6}{*}{ LOLBI } & $1-50$ & 23.8 & 32.0 & 40.2 & 9.5 \\
\hline & $51-100$ & 23.6 & 28.7 & 47.2 & 24.0 \\
\hline & $101-150$ & 28.3 & 26.5 & 29.2 & 36.0 \\
\hline & $151-200$ & 28.3 & 32.6 & 32.6 & 39.0 \\
\hline & MEAN & 26.0 & 30.0 & 37.3 & 27.1 \\
\hline & SDT DEV & 2.7 & 2.9 & 8.0 & 13.4 \\
\hline \multirow[t]{6}{*}{ LORBI } & $1-50$ & 34.3 & 42.8 & 24.0 & 6.9 \\
\hline & $51-100$ & 36.0 & 23.2 & 40.3 & 10.0 \\
\hline & $101-150$ & 36.8 & 21.4 & 40.3 & 23.5 \\
\hline & $151-200$ & 41.0 & 28.4 & 28.3 & 30.9 \\
\hline & MEAN & 37.0 & 29.0 & 33.2 & 17.8 \\
\hline & SDT DEV & 2.8 & 9.7 & 8.4 & 11.3 \\
\hline \multirow[t]{6}{*}{ LOLGI } & $1-50$ & 21.3 & 18.1 & 36.8 & 63.5 \\
\hline & $51-100$ & 22.3 & 22.3 & 35.1 & 64.3 \\
\hline & $101-150$ & 22.3 & 17.8 & 37.1 & 47.1 \\
\hline & $151-200$ & 9.4 & 23.1 & 41.2 & 45.1 \\
\hline & MEAN & 18.8 & 20.3 & 37.6 & 55.0 \\
\hline & STD DEV & 6.3 & 2.8 & 2.6 & 10.3 \\
\hline \multirow[t]{6}{*}{ LORGI } & $1-50$ & 16.4 & 20.5 & 42.0 & 33.5 \\
\hline & $51-100$ & 11.1 & 27.7 & 33.2 & 35.1 \\
\hline & $101-150$ & 10.3 & 18.8 & 42.9 & 26.5 \\
\hline & $151-200$ & 12.8 & 15.5 & 43.7 & 23.1 \\
\hline & MEAN & 12.7 & 20.6 & 40.5 & 29.6 \\
\hline & STD DEV & 2.7 & 5.2 & 4.9 & 5.7 \\
\hline
\end{tabular}

Table 6.13a Interpeak latencies from the main components of SUBJECT LO first VEP response using both stimulators. 


\begin{tabular}{|c|c|c|c|c|c|}
\hline Subject & Sweeps & P50-N75 & N75-P100 & P100-N145 & $\mathrm{N} 145-\mathrm{P} 175$ \\
\hline Code & & LAT msec & LAT msec & LAT msec & LAT msec \\
\hline \multirow[t]{6}{*}{ LOLB2 } & $1-50$ & 20.6 & 48.0 & 12.8 & 27.5 \\
\hline & $51-100$ & 18.0 & 41.2 & 23.1 & 13.7 \\
\hline & $101-150$ & 16.3 & 48.9 & 22.2 & 34.3 \\
\hline & $151-200$ & 16.6 & 51.4 & 18.9 & 30.0 \\
\hline & MEAN & 17.9 & 47.4 & 19.3 & 26.4 \\
\hline & SDT DEV & 2.0 & 4.4 & 4.7 & 8.9 \\
\hline \multirow[t]{6}{*}{ LORB2 } & $1-50$ & 33.4 & 42.0 & 14.6 & 34.3 \\
\hline & $51-100$ & 25.4 & 35.5 & 30.8 & 30.9 \\
\hline & $101-150$ & 43.2 & 15.9 & 30.9 & 33.4 \\
\hline & $151-200$ & 17.1 & 45.5 & 25.7 & 32.6 \\
\hline & MEAN & 29.8 & 34.7 & 25.5 & 32.8 \\
\hline & SDT DEV & 11.2 & 13.2 & 7.7 & 1.4 \\
\hline \multirow[t]{6}{*}{ LOLG2 } & $1-50$ & 19.7 & 18.9 & 24.0 & 81.4 \\
\hline & $51-100$ & 18.8 & 18.0 & 34.3 & 48.0 \\
\hline & $101-150$ & 20.5 & 14.6 & 34.3 & 49.7 \\
\hline & $151-200$ & 12.9 & 23.1 & 37.7 & 62.6 \\
\hline & MEAN & 18.0 & 18.7 & 32.6 & 60.4 \\
\hline & STD DEV & 3.5 & 3.5 & 5.9 & 15.4 \\
\hline \multirow[t]{6}{*}{ LORG2 } & $1-50$ & 24.8 & 25.7 & 36.0 & 42.3 \\
\hline & $51-100$ & 24.0 & 32.6 & 37.7 & 30.9 \\
\hline & $101-150$ & 21.5 & 31.7 & 30.8 & 36.0 \\
\hline & $151-200$ & 24.8 & 36.9 & 32.6 & 31.5 \\
\hline & MEAN & 23.8 & 31.7 & 34.3 & 35.2 \\
\hline & STD DEV & 1.6 & 4.6 & 3.1 & 5.3 \\
\hline
\end{tabular}

Table $6.13 \mathrm{~b}$ Interpeak latencies from the main components of SUBJECT LO first VEP response using both stimulators. 


\begin{tabular}{|c|c|c|c|c|c|}
\hline Subject & Sweeps & P50-N75 & N75-P100 & P100-N145 & N145-P175 \\
\hline Code & & LAT msec & LAT msec & LAT msec & LAT msec \\
\hline \multirow[t]{6}{*}{ MSLBI } & $1-50$ & 12.9 & 42.8 & 30.0 & 24.0 \\
\hline & $51-100$ & 19.8 & 41.1 & 23.1 & 30.0 \\
\hline & $101-150$ & 24.0 & 45.4 & 29.2 & 29.1 \\
\hline & $151-200$ & 24.0 & 43.7 & 30.0 & 25.7 \\
\hline & MEAN & 20.2 & 43.3 & 28.1 & 27.2 \\
\hline & SDT DEV & 5.2 & 1.8 & 3.3 & 2.8 \\
\hline \multirow[t]{6}{*}{ MSRBI } & $1-50$ & 19.7 & 38.6 & 25.7 & 24.0 \\
\hline & $51-100$ & 19.8 & 39.4 & 30.0 & 31.7 \\
\hline & $101-150$ & 20.6 & 42.0 & 23.2 & 21.4 \\
\hline & $151-200$ & 24.0 & 36.0 & 23.2 & 18.0 \\
\hline & MEAN & 21.0 & 39.0 & 25.5 & 23.8 \\
\hline & SDT DEV & 2.0 & 2.5 & 3.2 & 5.8 \\
\hline \multirow[t]{6}{*}{ MSLG1 } & $1-50$ & 17.1 & 19.7 & 50.6 & 37.7 \\
\hline & $51-100$ & 14.6 & 18.0 & 50.6 & 45.4 \\
\hline & $101-150$ & 27.4 & 21.5 & 46.3 & 50.5 \\
\hline & $151-200$ & 28.3 & 18.9 & 47.1 & 44.6 \\
\hline & MEAN & 21.9 & 19.5 & 48.7 & 44.6 \\
\hline & STD DEV & 7.0 & 1.5 & 2.3 & 5.3 \\
\hline \multirow[t]{6}{*}{ MSRGI } & $1-50$ & 13.7 & 22.3 & 49.7 & 42.0 \\
\hline & $51-100$ & 19.7 & 17.2 & 51.4 & 38.6 \\
\hline & $101-150$ & 23.1 & 18.9 & 40.3 & 39.4 \\
\hline & $151-200$ & 23.1 & 16.3 & 51.5 & 48.8 \\
\hline & MEAN & 19.9 & 18.7 & 48.2 & 42.2 \\
\hline & STD DEV & 4.4 & 2.6 & 5.3 & 4.6 \\
\hline
\end{tabular}

Table 6.14a Interpeak latencies from the main components of SUBJECT MS first VEP response using both stimulators. 


\begin{tabular}{|c|c|c|c|c|c|}
\hline Subject & Sweeps & P50-N75 & N75-P100 & P100-N145 & N145-P175 \\
\hline Code & & LAT msec & LAT msec & LAT msec & LAT msec \\
\hline \multirow[t]{6}{*}{ MSLB2 } & $1-50$ & 17.2 & 32.5 & 24.9 & 30.0 \\
\hline & $51-100$ & 12.8 & 33.5 & 27.4 & 31.0 \\
\hline & $101-150$ & 18.9 & 33.4 & 27.3 & 31.0 \\
\hline & $151-200$ & 14.6 & 16.3 & 42.0 & 30.8 \\
\hline & MEAN & 15.9 & 28.9 & 30.4 & 30.7 \\
\hline & SDT DEV & 2.7 & 8.4 & 7.8 & 0.5 \\
\hline \multirow[t]{6}{*}{ MSRB2 } & 1.50 & 11.2 & 13.7 & 42.0 & 38.7 \\
\hline & $51-100$ & 12.9 & 24.8 & 30.9 & 35.1 \\
\hline & $101-150$ & 11.3 & 17.1 & 36.2 & 36.1 \\
\hline & $151-200$ & 10.3 & 17.1 & 40.3 & 35.2 \\
\hline & MEAN & 11.4 & 18.2 & 37.4 & 36.3 \\
\hline & SDT DEV & 1.1 & 4.7 & 4.9 & 1.7 \\
\hline \multirow[t]{6}{*}{ MSLG2 } & $1-50$ & 17.1 & 18.9 & 52.3 & 41.8 \\
\hline & $51-100$ & 14.7 & 18.8 & 50.0 & 41.7 \\
\hline & $101-150$ & 14.5 & 20.6 & 48.9 & 40.3 \\
\hline & $151-200$ & 14.5 & 19.7 & 47.2 & 37.7 \\
\hline & MEAN & 15.2 & 19.5 & 49.6 & 40.4 \\
\hline & STD DEV & 1.3 & 0.8 & 2.1 & 1.9 \\
\hline \multirow[t]{6}{*}{ MSRG2 } & $1-50$ & 21.4 & 18.9 & 46.3 & 41.1 \\
\hline & $51-100$ & 19.7 & 42.0 & 27.4 & 46.6 \\
\hline & $101-150$ & 20.6 & 19.7 & 46.3 & 41.1 \\
\hline & $151-200$ & 14.6 & 24.8 & 43.7 & 45.3 \\
\hline & MEAN & 19.1 & 26.4 & 40.9 & 43.5 \\
\hline & STD DEV & 3.1 & 10.8 & 9.1 & 2.9 \\
\hline
\end{tabular}

Table 6.14b Interpeak latencies from the main components of SUBJECT MS first VEP response using both stimulators. 


\begin{tabular}{|c|c|c|c|c|c|}
\hline Subject & Sweeps & P50-N75 & N75-P100 & P100-N145 & N145-P175 \\
\hline Code & & LAT msec & LAT msec & LAT msec & LAT msec \\
\hline \multirow[t]{6}{*}{ RDLBI } & $1-50$ & 34.3 & 14.6 & 46.3 & 19.0 \\
\hline & $51-100$ & 24.9 & 15.4 & 38.6 & 48.0 \\
\hline & $101-150$ & 22.3 & 11.2 & 47.1 & 37.7 \\
\hline & $151-200$ & 28.3 & 12.9 & 54.0 & 51.4 \\
\hline & MEAN & 27.5 & 13.5 & 46.5 & 39.0 \\
\hline & SDT DEV & 5.2 & 1.9 & 6.3 & 14.6 \\
\hline \multirow[t]{6}{*}{ RDRBI } & $1-50$ & 18.0 & 35.1 & 47.2 & 24.8 \\
\hline & $51-100$ & 6.8 & 29.2 & 54.8 & 24.9 \\
\hline & $101-150$ & 7.1 & 27.4 & 52.3 & 38.6 \\
\hline & $151-200$ & 8.2 & 34.1 & 53.1 & 43.7 \\
\hline & MEAN & 10.0 & 31.5 & 51.9 & 33.0 \\
\hline & SDT DEV & 5.4 & 3.7 & 3.3 & 9.6 \\
\hline \multirow[t]{6}{*}{ RDLG1 } & $1-50$ & 21.4 & 22.3 & 36.0 & 49.4 \\
\hline & $51-100$ & 20.6 & 22.2 & 49.8 & 28.2 \\
\hline & $101-150$ & 17.2 & 16.3 & 49.7 & 36.0 \\
\hline & $151-200$ & 32.5 & 11.2 & 54.8 & 35.2 \\
\hline & MEAN & 22.9 & 18.0 & 47.6 & 37.2 \\
\hline & STD DEV & 6.6 & 5.3 & 8.1 & 8.9 \\
\hline \multirow[t]{6}{*}{ RDRG1 } & $1-50$ & 16.3 & 31.7 & 48.8 & 26.6 \\
\hline & $51-100$ & 17.1 & 35.2 & 48.8 & 46.3 \\
\hline & $101-150$ & 18.9 & 24.9 & 55.7 & 32.5 \\
\hline & $151-200$ & 17.1 & 30.9 & 54.0 & 33.4 \\
\hline & MEAN & 17.4 & 30.7 & 51.8 & 34.7 \\
\hline & STD DEV & 1.1 & 4.3 & 3.6 & 8.3 \\
\hline
\end{tabular}

Table 6.15a Interpeak latencies from the main components of SUBJECT RD first VEP response using both stimulators. 


\begin{tabular}{|c|c|c|c|c|c|}
\hline Subject & Sweeps & P50-N75 & N75-P100 & P100-N145 & N145-P175 \\
\hline Code & & LAT msec & IAT msec & LAT msec & LAT msec \\
\hline \multirow[t]{6}{*}{ RDLB2 } & $1-50$ & 10.8 & 28.3 & 51.4 & 45.5 \\
\hline & $51-100$ & 20.5 & 18.0 & 52.3 & 35.2 \\
\hline & $101-150$ & 10.3 & 24.0 & 55.7 & 45.5 \\
\hline & $151-200$ & 11.3 & 24.7 & 57.4 & 45.4 \\
\hline & MEAN & 13.2 & 23.8 & 54.2 & 42.9 \\
\hline & SDT DEV & 4.9 & 4.3 & 2.8 & 5.1 \\
\hline \multirow[t]{6}{*}{ RDRB2 } & $1-50$ & 24.0 & 24.0 & 44.6 & 45.4 \\
\hline & $51-100$ & 18.0 & 21.4 & 42.9 & 49.7 \\
\hline & $101-150$ & 19.7 & 14.6 & 47.1 & 47.2 \\
\hline & $151-200$ & 24.0 & 13.7 & 50.1 & 45.0 \\
\hline & MEAN & 21.4 & 18.4 & 46.2 & 46.8 \\
\hline & SDT DEV & 3.1 & 5.1 & 3.1 & 2.1 \\
\hline \multirow[t]{6}{*}{ RDLG2 } & $1-50$ & 12.0 & 15.4 & 54.9 & 40.3 \\
\hline & $51-100$ & 14.6 & 15.4 & 55.7 & 41.2 \\
\hline & $101-150$ & 12.9 & 15.4 & 54.9 & 41.0 \\
\hline & $151-200$ & 16.3 & 15.4 & 52.3 & 39.4 \\
\hline & MEAN & 14.0 & 15.4 & 54.5 & 40.5 \\
\hline & STD DEV & 1.9 & 0.0 & 1.5 & 0.8 \\
\hline \multirow[t]{6}{*}{ RDRG2 } & $1-50$ & 8.5 & 19.8 & 50.5 & 38.6 \\
\hline & $51-100$ & 13.7 & 18.0 & 53.7 & 40.6 \\
\hline & $101-150$ & 9.7 & 22.0 & 53.2 & 37.7 \\
\hline & $151-200$ & 9.8 & 26.6 & 53.2 & 36.0 \\
\hline & MEAN & 10.4 & 21.6 & 52.7 & 38.2 \\
\hline & STD DEV & 2.3 & 3.7 & 1.5 & 1.9 \\
\hline
\end{tabular}

Table $6.15 \mathrm{~b}$ Interpeak latencies from the main components of SUBJECT RD first VEP response using both stimulators. 
Interpeak latencies of the main VEP components were also compared with the Andersson's LED-Box stimulator. The results are shown in the following figure:

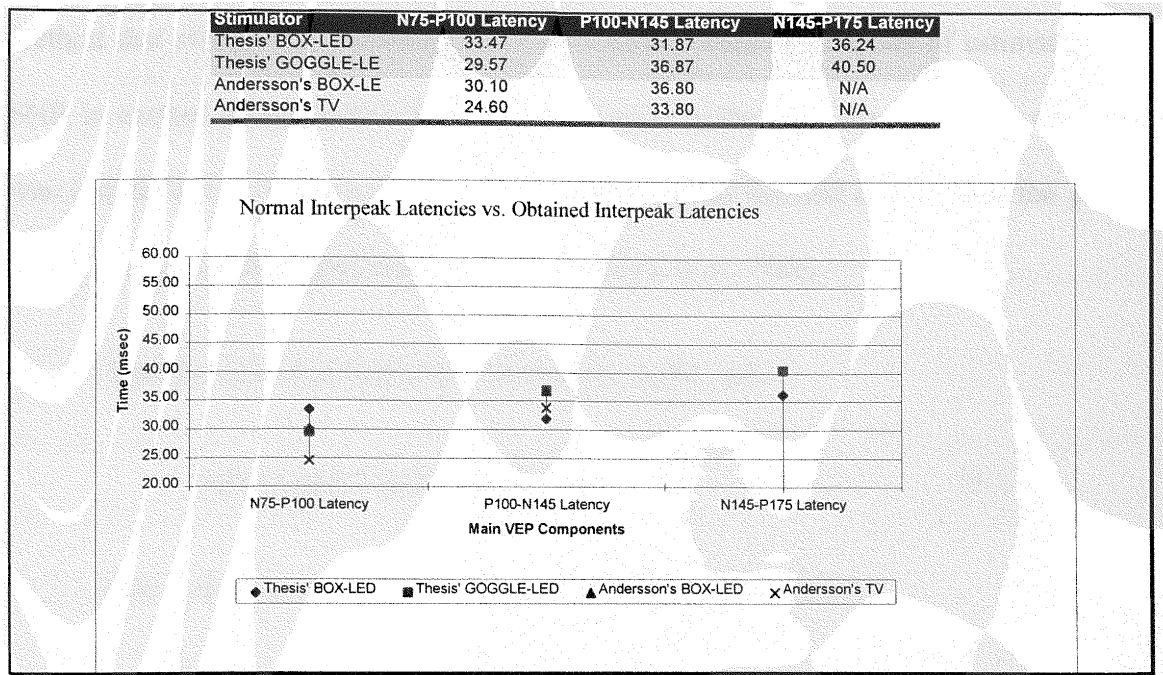

Figure 6.10 A graphical representation of the interpeak latencies obtained from the LEDGoggle and LED-Box versus the Andersson's LED-Box and TV monitor.

With the LED-goggle, the N75-P100 interpeak latency was found to be 3.9 milli-seconds shorter than in the LED-box. However, P100-N145 and N145-P175 were found to be 5 and 4.26 milli-seconds shorter with respect to the LED-goggle interpeak latencies. On the other hand, there were no significant differences in interpeak latencies in N75 and P100 for any of the four stimulators, which confirms the appropriateness of the measurements obtained. 


\subsection{Signal to Noise Ratio Analysis}

Signal-to-noise ratio (SNR) estimation technique is an objective, quantitative method that effectively determines the VEP quality. Various methods of estimating the SNR in evoked potential data have been developed in the past. An alternative approach was introduced by Möck et al. in 1984. This particular method is based on the power estimate ratios of the signal and the noise (Özdamar et al. 1995).

The post-stimulus response is denoted by $\mathbf{x}_{\mathbf{k}}(\mathbf{t})$, and

$$
x_{k}(t)=s_{k}(t)+n_{k}(t), \text { where }
$$

$\mathrm{s}_{\mathrm{k}}(\mathrm{t})$ is the post-stimulus VEP signal (assumed deterministic);

$\mathrm{n}_{\mathrm{k}}(\mathrm{t})$ is the post-stimulus noise (assumed random);

$\mathrm{k}$ is the number of sweeps averaged.

Let us assume that $\mathrm{s}(\mathrm{t})$ is a deterministic signal and is the same for every stimulus; then

$$
\begin{aligned}
& \mathrm{s}_{\mathrm{k}}(\mathrm{t})=\mathrm{s}(\mathrm{t}) \text { and the averaging result is } \\
& \bar{x}(\mathrm{t})=\frac{1}{K} \sum_{k} x_{k}(t)=\mathrm{s}(\mathrm{t})+\frac{1}{K} \sum_{k} n_{k}(t)
\end{aligned}
$$

Therefore, the signal power $P_{s}$ and the noise power $P_{n}$ for each particular sweep are defined as following: 


$$
\begin{array}{ll}
\mathrm{P}_{\mathrm{s}}(\mathrm{k})=\sum\left[\left(\mathrm{s}(\mathrm{t})-\mu_{\mathrm{s}}\right)^{2}\right]=\sigma_{k}^{2} & \text { (Signal variance) } \\
\mathrm{P}_{\mathrm{n}}(\mathrm{k})=\sum\left[\left(\mathrm{n}(\mathrm{t})-\mu_{\mathrm{N}}\right)^{2}\right]=\sigma_{n}^{2}(\mathrm{k}) & \text { (noise variance) }
\end{array}
$$

Due to the band-pass filtering implicit in EEG recordings, the mean value of the signal $\mu_{\mathrm{s}}$, and the mean value of the noise $\mu_{\mathrm{N}}$, are generally negligible (Özdamar et al. 1995). Noise power is dependent on the EEG characteristics of the subject, which is mostly stationary and cannot be reduced. On the other hand, other electromagnetic interfering signals such a $60 \mathrm{~Hz}$ power signal and electromylogram (EMG) can be reduced.

The signal power, $\mathrm{P}_{\mathrm{s}}$, is primarily dependent on neuron generators and can be altered by changing various experimental conditions such as stimulus parameters, and electrode montage. Because of the zero-mean assumption for the noise and the timelocked nature of $s(t)$ with respect to the stimulus, the SNR can be expected to improve as a progressively large number of the time-aligned sweeps are averaged. Under these assumptions, the SNR is a function of the number of sweeps averaged and can be expressed as follows:

$$
\operatorname{SNR}(\mathrm{k})=\mathrm{k} \sigma_{k}^{2} / \sigma_{n}^{2}
$$


Theoretically, the signal power, $\mathrm{P}_{\mathrm{s}}$, stays the same for all k. On the other hand, residual noise power decreases by the factor of $1 / \mathrm{k}$ as $\mathrm{k}$ increases. Therefore, SNR is a linear function of $\mathrm{k}$ with a slope equal to $\sigma_{k}^{2} / \sigma_{n}^{2}$. However, under actual recording conditions, the signal and noise power vary (in some cases substantially) from one sweep to another.

The noise power can be estimated from the following equation:

$$
\hat{P}_{n}=\hat{\sigma}^{2}{ }_{n}=\frac{1}{T K} \cdot \sum_{t}\left(\sum_{k}(x(t)-\bar{x}(t))^{2}\right)
$$

where the residual noise power is estimated after averaging as follows:

$$
\hat{P}=\frac{1}{T K^{2}} \cdot \sum_{t}\left(\sum_{k}(x(t)-\bar{x}(t))^{2}\right)
$$

Therefore, the signal power is finally estimated by the averaged signal power minus the noise power as follows:

$$
\bar{P}_{s}=\hat{P}_{\bar{x}}-\hat{P}_{\bar{n}}=\hat{\sigma}_{\bar{x}}^{2}-\hat{\sigma}_{\bar{n}}^{2}
$$

Recalling that the averaged signal power is given by the following equation:

$$
\begin{aligned}
& \hat{P}_{\bar{x}}=\frac{1}{T} \cdot \sum_{t}\left(\bar{x}(t)-\mu_{\bar{x}}\right)^{2}, \text { assuming that } \mu_{\bar{x}}=0 \text { then } \\
& \hat{P}_{\bar{x}}=\frac{1}{T} \cdot \sum_{t}(\bar{x}(t))^{2}
\end{aligned}
$$


Then the SNR estimate can be represented as follows:

$$
\hat{S N R} \bar{x}=\frac{\hat{P_{\bar{x}}}-\hat{P}_{\bar{n}}}{\hat{P_{\bar{n}}}}=\frac{\hat{P_{\bar{x}}}}{\hat{P}_{\bar{n}}}-
$$

If $P_{\bar{x}}$ approaches zero then $S N R_{\bar{x}}$ approaches -1 . The above equation cannot be computed easily in real-time during a running average since a large number of operations would be required and the huge memory storage is required to save all the single responses in memory. Therefore, these problems can be solved by deriving computational formulas which require only the storage of sums and squared sums for each time point (Özdamar et al. 1995).

A computational formula for $\hat{P}_{\bar{n}}$ was derived by Özdamar et al., by using the following expectation equation (with respect to $\mathrm{k}$ ):

$$
E_{k}\left[\left(x_{k}(t)-\bar{x}(t)\right)^{2}\right]=E_{k}\left[\left(x_{k}(t)\right]^{2}-\left(E_{k}[\bar{x}(t)]\right)^{2}\right.
$$

Therefore, the residual noise estimate can be expressed as

$$
\hat{P}_{\bar{n}}=\frac{1}{T K^{2}} \sum_{t}\left(\sum_{k} x_{k}^{2}(t)-\frac{1}{K}\left(\sum_{k} x_{k}(t)\right)^{2}\right)
$$

Then equation (6.3.9) and the above equation can be substituted into equation (6.3.8) and the simplified result is as follows: 


$$
\hat{P}_{s}=\left(\frac{1}{T K^{2}}\left(1+\frac{1}{K}\right)\right) \cdot \sum_{t}\left(\sum_{k} x_{k}(t)\right)^{2}-\frac{1}{T K^{2}}\left[\cdot \sum_{t}\left(\sum_{k} x_{k}^{2}(t)\right)\right]
$$

Substituting the above equation into equation (6.3.10) and further simplification, then the SNR estimate can be expressed as follows:

$$
\hat{S N R}=\frac{\left(1+\frac{1}{K}\right) \cdot\left(\sum_{t}\left(\sum_{k} x_{k}(t)\right)^{2}-\sum_{t}\left(\sum_{k} x_{k}^{2}(t)\right)\right.}{\sum_{t}\left(\sum_{k} x_{k}^{2}(t)\right)-\frac{1}{K} \cdot \sum_{t}\left(\sum_{k} x_{k}(t)\right)^{2}}(\text { Özdamar et al. 1995). }
$$

A running SNR estimation program, written in Pascal, was developed. The running SNR program calculated the pre-stimulus and the post-stimulus portions of the recorded EEG. Figure 6.17 shows the flow diagram of this estimation program. A maximum of 200 sweeps was acquired for each recording. Figure 6.12 shows a typical post-stimulus and pre-stimulus SNR estimates for the LED-Box and Figure 6.13 shows the SNR estimates for the LED-Goggle on the same subject. 


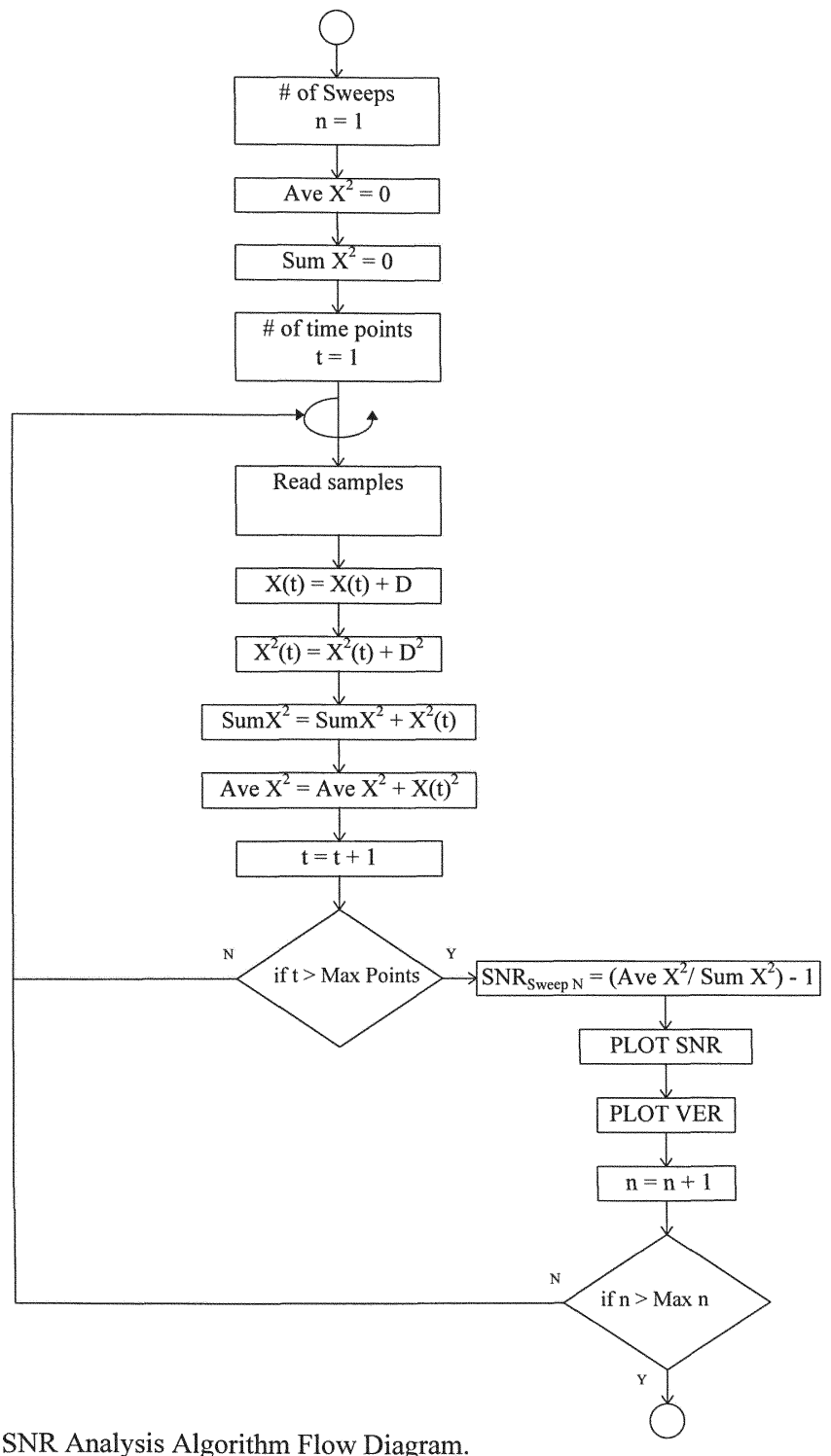

Figure 6.11 SNR Analysis Algorithm Flow Diagram. 
Left Eye

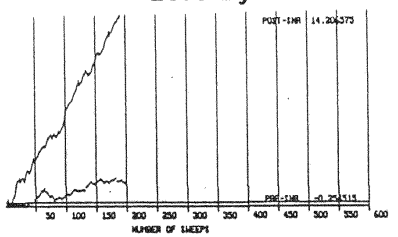

Sweeps

25

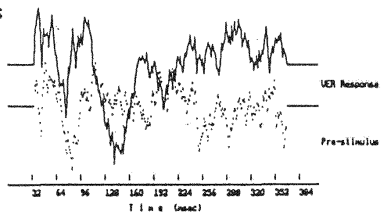

50
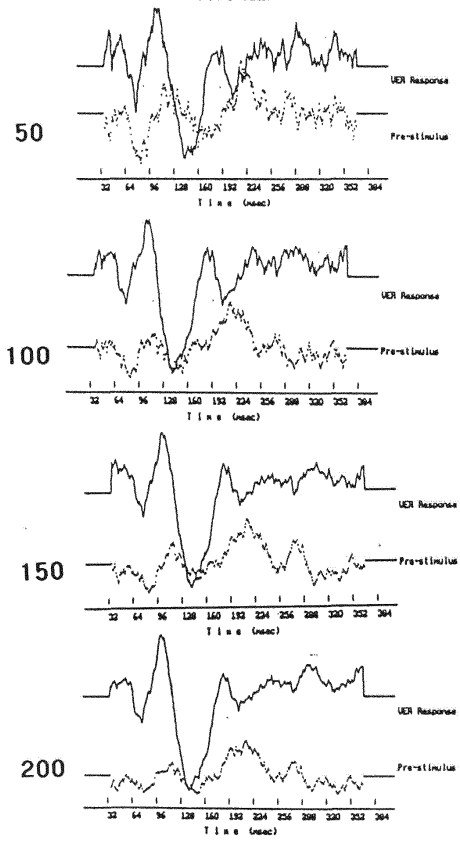

Right Eye
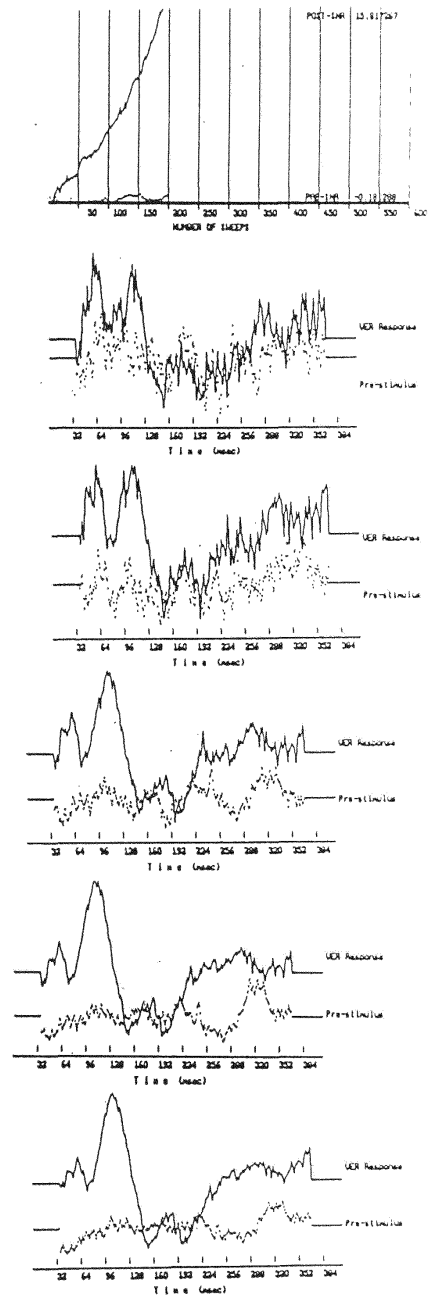

Figure 6.12 Pre- and post-stimulus SNR estimate (top graph) of a recording acquired with the LED-Box checkerboard stimulator. Average response after 25, 50, 100, 150, and 200 sweeps (bottom graphs) 

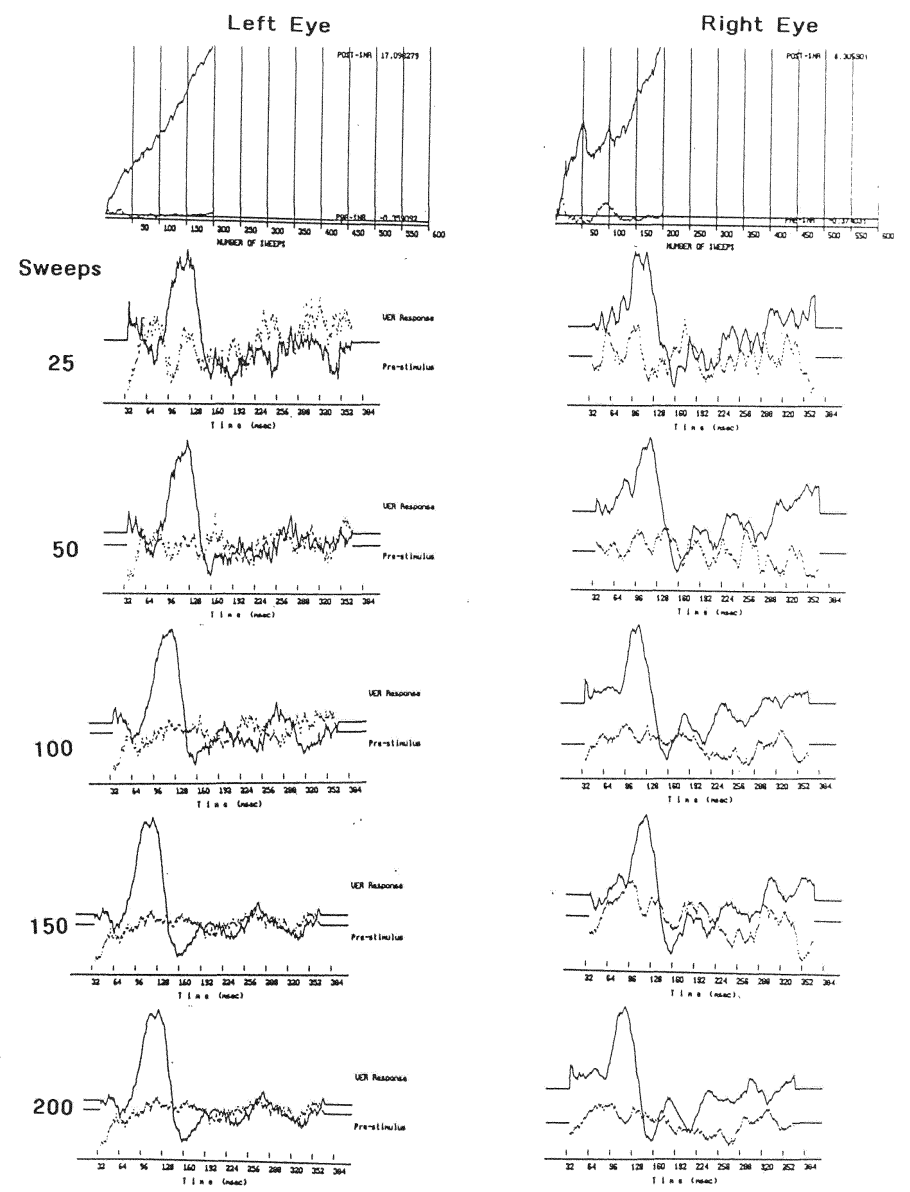

Figure 6.13 Pre- and post-stimulus SNR estimate (top graph) of a recording acquired with the LED- Goggle checkerboard stimulator. Average response after 25, 50,100, 150, and 200 sweeps (bottom graphs) 
The overall SNR calculations obtained from two recordings of three subjects are shown in Figures 6.14 through 6.16. These results shown the post-stimulus and the pre-stimulus SNR calculations obtained with 400 sweeps from both stimulators.
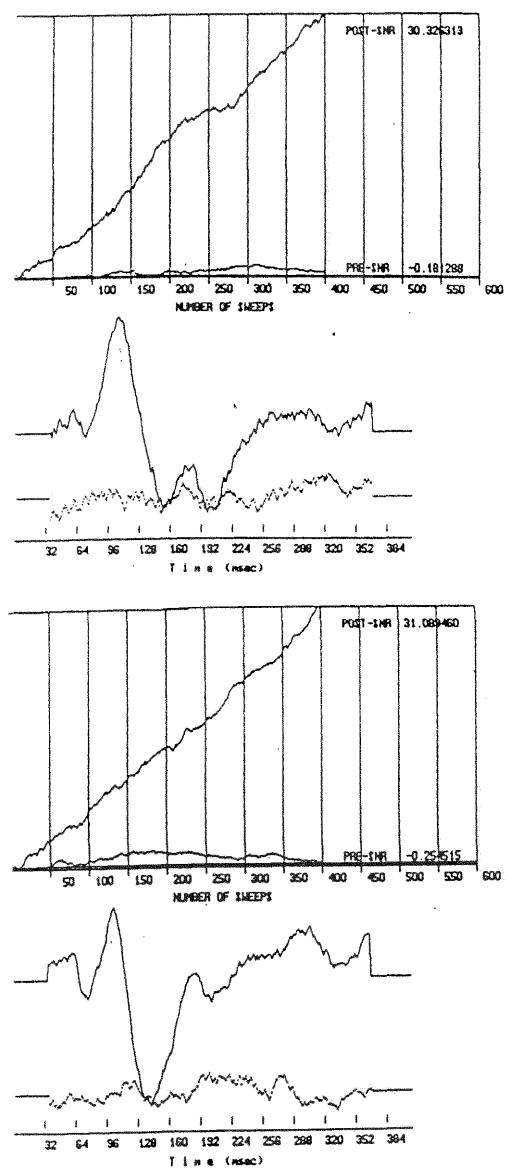
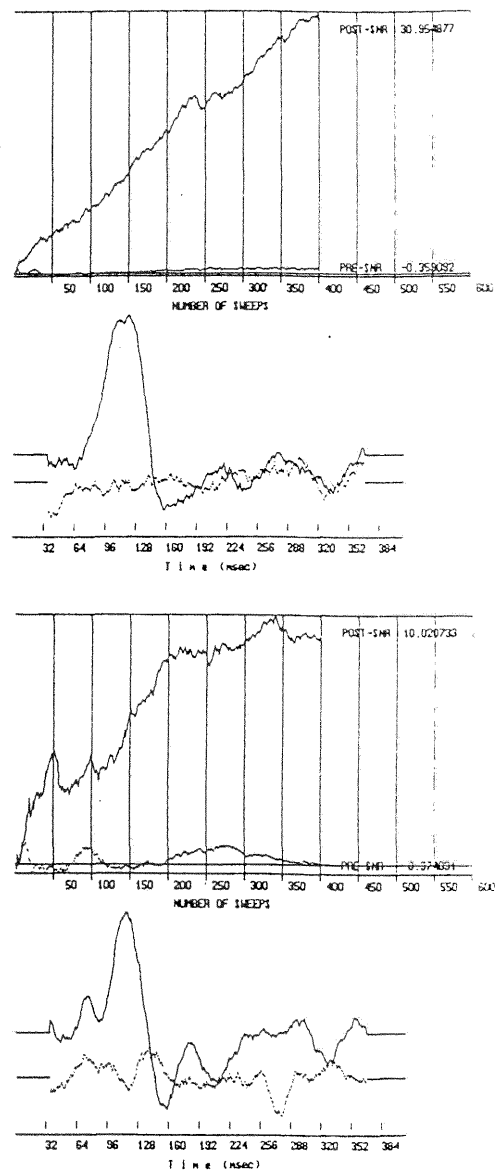

Figure 6.14 SNR calculations and its average VEP responses from subject CQ. The top and bottom recording from the left hand side correspond to the LED-Box recordings. The top and bottom right hand side recording correspond to the LED-Goggle recordings. 

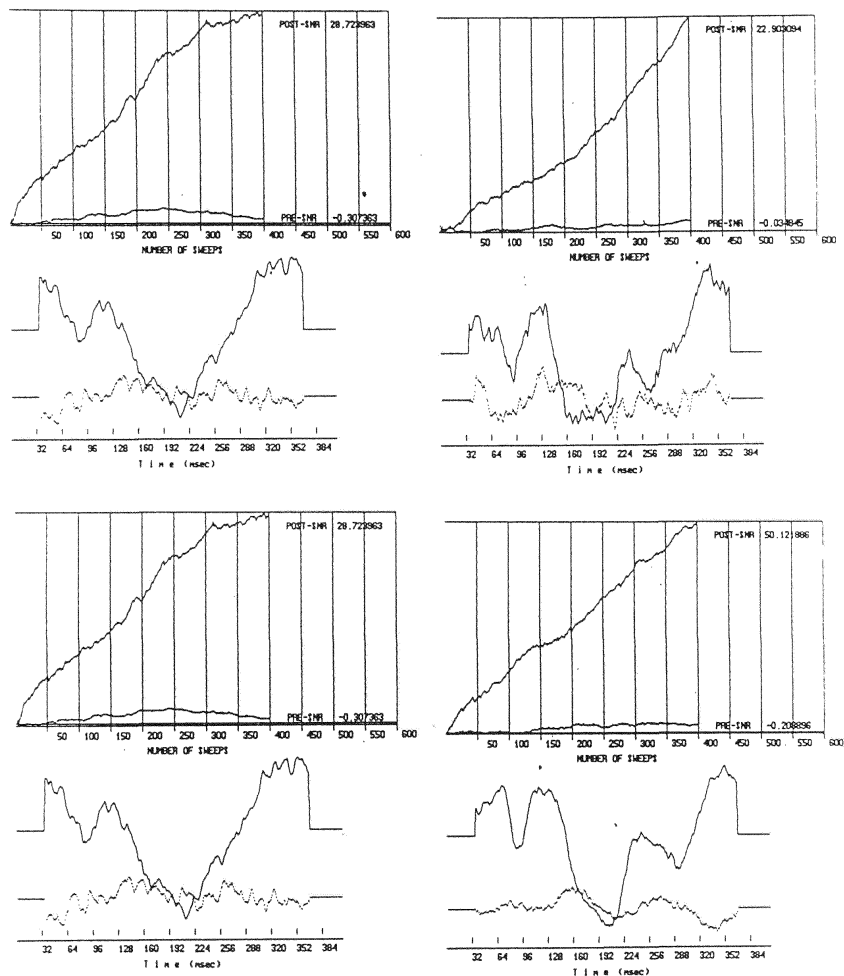

Figure 6.15 SNR calculations and its average VEP responses from subject LO. The top and bottom recording from the left hand side correspond to the LED-Box recordings. The top and bottom right hand side recording correspond to the LED-Goggle recordings. 

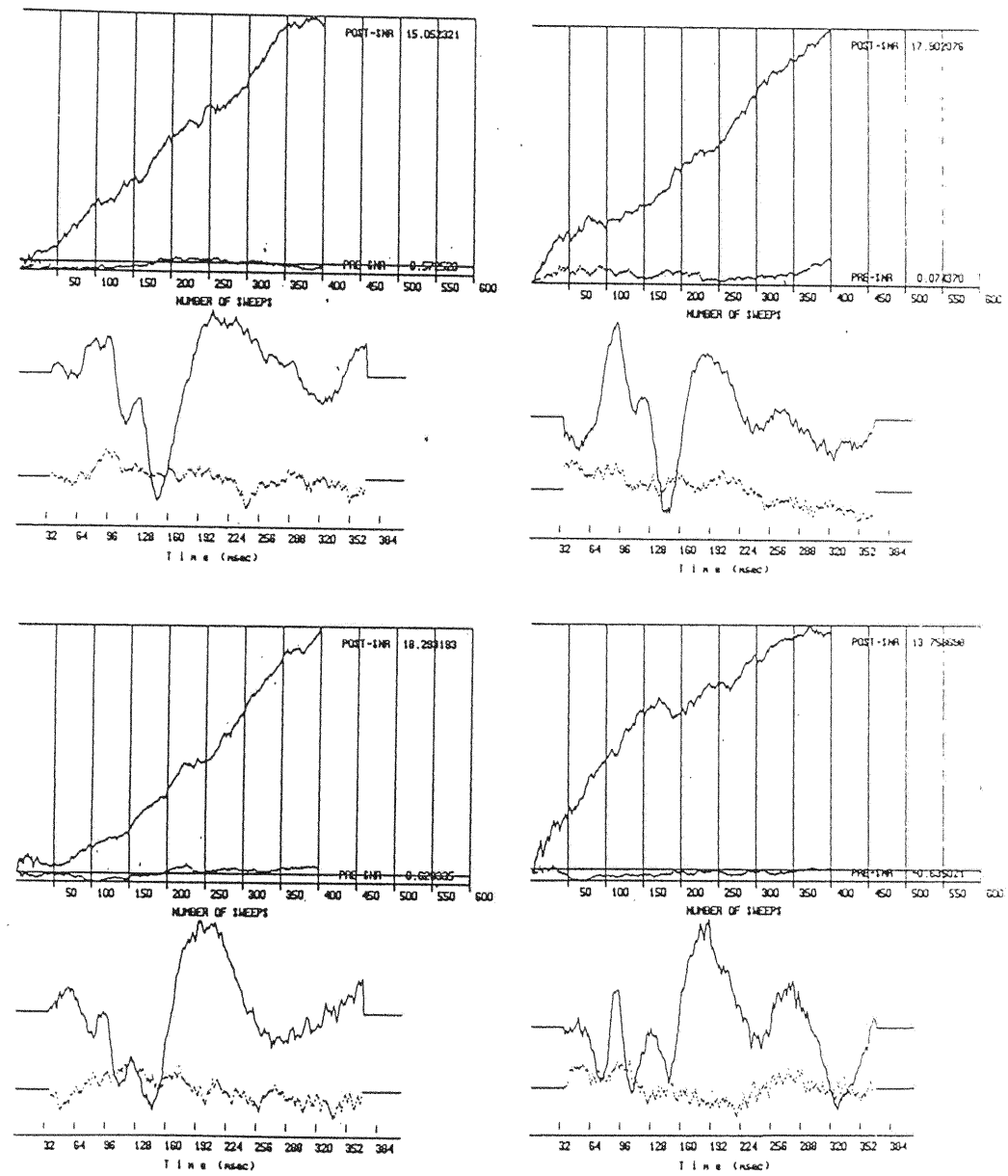

Figure 6.16 SNR calculations and its average VEP responses from subject RD. The top and bottom recording from the left hand side correspond to the LED-Box recordings. The top and bottom right hand side recording correspond to the LED-Goggle recordings. 


\subsection{Cross correlation Analysis}

Besides the SNR estimation, a windowed cross-correlation between two sequential recordings acquired at the same stimulation check size was also calculated. Cross correlation operations are used extensively in signal and statistical analysis, both in continuous-time systems and discrete-time systems. In this context, the cross correlation between consecutive recordings indicates the repeatability, and therefore, the reliability of the measurement. Correlation applications can be achieved with either a general-purpose computer or with a special-purpose digital processor using discrete-time techniques. For the sake of simplicity, the basic mathematical cross correlation relationships will first be discussed in terms of their discrete-time forms. The cross correlation coefficient $\left(\mathrm{R}_{\mathrm{xy}}\right)$ is calculated using the equation shown below:

$$
\begin{aligned}
& \mathrm{R}_{\mathrm{xy}}= \sum_{j-1}^{N i}\left[\left(X_{i}(j)-m_{x i}\right) \cdot\left(Y_{i}(j)-m_{y i}\right)\right] \\
& \sqrt{\sum_{j-1}^{N i}\left(X_{i}(j)-m_{x i}\right)^{2}} \cdot \sqrt{\sum_{j-1}^{N i}\left(Y_{i}(j)-m_{y i}\right)^{2}}
\end{aligned}
$$

where $\mathrm{X}_{\mathrm{i}}$ corresponds to the data points of the first recording, $\mathrm{Y}_{\mathrm{i}}$ corresponds to the data points of the second recording, $\mathrm{m}_{\mathrm{xi}}$ and $\mathrm{m}_{\mathrm{yi}}$ correspond to the mean values, and $\mathrm{N}_{\mathrm{i}}$ corresponds to the number of points. The value of $\mathrm{R}_{\mathrm{xy}}$ indicates how well two signals are correlated for that particular value. If the correlation has the value of 1 , then indicates 
a very good correlation, which means that the two signals match each other very well. Conversely, a very small or zero value indicates little or no correlation (Stanley, 1984).

A computational formula was derived from the above equation. A 200 milliseconds window was selected to calculate the cross correlation of the two averaged recordings starting at 40 milli-seconds to 240 milli-seconds. Table 6.16 shows the crosscorrelation calculation from two sequential recordings.

\begin{tabular}{|c|c|c|c|c|}
\hline $\begin{array}{c}\text { Subject } \\
\text { Code }\end{array}$ & $\begin{array}{c}\text { LED-Box } \\
\text { Right Eye } \\
\text { Cross- } \\
\text { Correlation }\end{array}$ & $\begin{array}{c}\text { LED-Box } \\
\text { Left Eye } \\
\text { Cross- } \\
\text { Correlation }\end{array}$ & $\begin{array}{c}\text { LED-Goggle } \\
\text { Right Eye } \\
\text { Cross- } \\
\text { Correlation }\end{array}$ & $\begin{array}{c}\text { LED-Goggle } \\
\text { Left Eye } \\
\text { Cross- } \\
\text { Correlation }\end{array}$ \\
\hline CQ & 0.94 & 0.97 & 0.80 & 0.88 \\
CL & 0.95 & 0.90 & 0.88 & 0.95 \\
IM & 0.89 & 0.88 & 0.91 & 0.97 \\
LO & 0.83 & 0.88 & 0.99 & 0.72 \\
MS & 0.88 & 0.72 & 0.91 & 0.72 \\
RD & 0.94 & 0.98 & 0.96 & 0.91 \\
\hline
\end{tabular}

Table 6.16 Cross Correlation results from VEP recordings using the LED-Box and LEDGoggle stimulators. 32' check size; full-field stimulation.

SNR estimation results indicated a slightly higher SNR estimate in the LEDGoggle than in the LED-Box in most of the collected data. The cross correlation values indicated high cross correlation result (at or above 0.72 ) for the LED-Box recordings. The average cross correlation value of the LED-Goggle was also ranging between 0.80 and 0.72 for left and right eye recordings respectively as shown in Figure 6.17.

The LED-box stimulator evoked similar potentials with equivalent latency and equal amplitude to those obtained with the LED-goggle. The LED-goggle stimulator can 
provide better peak amplitudes and morphology response. On the other hand, the LEDbox stimulator resulted in a better cross correlation of the recordings. However, the LEDgoggle stimulator resulted in a slightly higher SNR estimation in some of the recordings.

In summary, the overall VEP assessment provided by both stimulators in this study allows the clinician to use a new approach in stimulating VEPs. The ability of testing subjects using the same Evoked Potential acquisition system, but with two different stimulators, will improve the diagnostic results when both methods are combined.

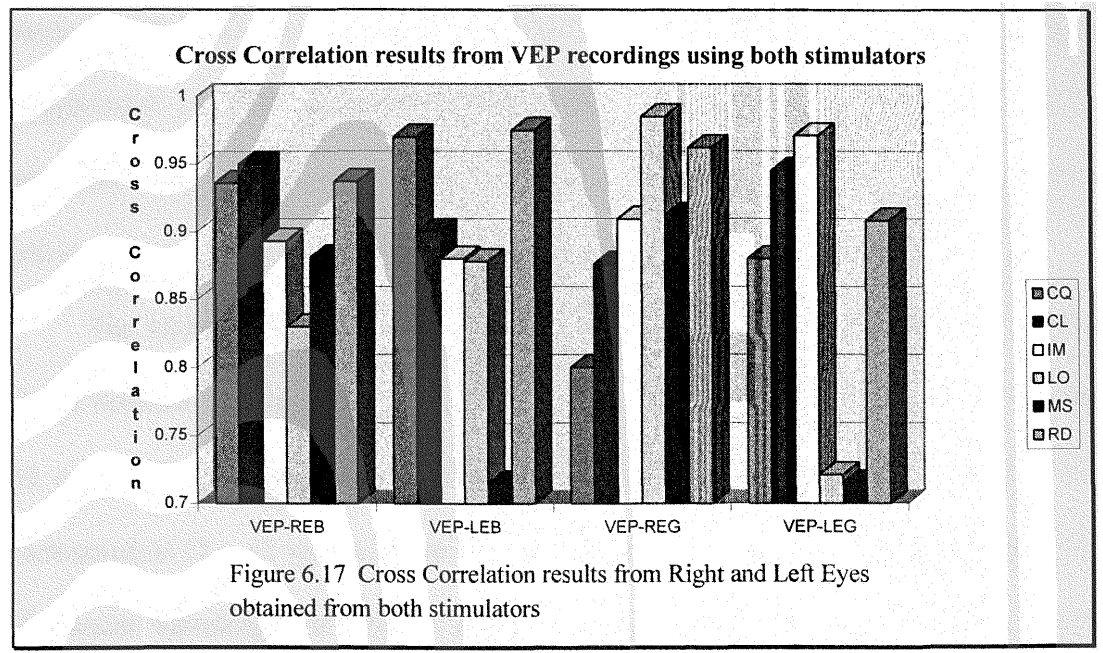




\section{CHAPTER 7 - DISCUSSION}

In this study, two computer-controlled pattern reversal checkerboard VEP stimulators were developed, evaluated, compared and their results presented. To the author's knowledge, there is no other pattern reversal VEP LED-goggle reported in the literature with the same capabilities as the one developed for this study.

\subsection{Comparison between stimulators}

A variety of checkerboard pattern reversal stimulators have been designed ranging from simple slide-projector to more complex designs such as television. However, TV monitors and LED are the most commonly used stimulators for diagnostic studies. Comparisons of VEPs elicited by LEDs and TV monitors have been performed previously. But comparison of VEPs elicited by two LEDs stimulators have not been performed.

The checkerboard LED-box is a versatile device allowing the user to perform pattern reversal checkerboard and bars; onset-offset checkerboard and bars at full, half, and quarter field stimulation, or even user-defined patterns. The LED patterns were constructed of $12 \times 12$ square LEDs. Each LED was 5 x $5 \mathrm{~mm}$ and emitting red light with a dominant wavelength of $650 \mathrm{~nm}$. A total of 144 resistors was needed to allow $10 \mathrm{~mA}$ through each LED. Furthermore, nine TMS27C210 EPROMs were needed to performed the patterns on the $12 \times 12$ array.

The LED-goggle was a similar design as the LED-box except that the LED patterns were constructed of two $8 \times 8$ square LEDs with each LED being $3 \times 3 \mathrm{~mm}$ and 
also emitting a red light with a wavelength of $635 \mathrm{~nm}$. Only 64 resistors and 4 TMS27PC10 PROMs were needed for each $8 \times 8$ array. One of the major differences was that the LED-goggle stimulator was constructed using surface mount (SMT) components which makes this stimulator smaller in size. One of the major advantages of the LEDgoggle stimulator was power consumption because fewer components were needed. Furthermore, other advantage of using the LED-goggle stimulator was that the clinician may stimulate each eye monocularly without occluding the other eye as in the LED-box stimulator. With respect to VEP responses, there were no significant differences in the VEP results between the proposed LED-goggle stimulator and the LED-Box. Although both stimulators' latency components were found to be slightly shorter comparing them with the results previously reported in the literature.

In summary, LED-goggle stimulation evoked VEP responses similar to the LEDbox. However, in several recordings, higher amplitudes were detected in some VEP components with the LED-goggle stimulator (e.g. larger N75 amplitude). 


\section{CHAPTER 8 - CONCLUSION}

\subsection{Accomplishments}

In this study two computer-controlled pattern reversal VEP stimulators were developed, evaluated, and compared. These results demonstrate that a different approach of stimulating VEP response can be used as an alternative method to verify the response absolute latencies and amplitudes, as well as, the interpeak latencies in the same subject. The study also demonstrated that a better result can be obtained when both stimulators are used.

\subsection{Future Developments}

The LED-box stimulator is currently in used at the University of Miami, Biomedical Department, where more volunteers will be tested using the same technique and protocol as was done in this thesis. Future study will be done to observe the change of latency and amplitude of the major components of the VEP responses using other patterns (bars, onset-offset checkerboard, etc.), colors (red, green, orange, etc.), and agerelated effects.

A new computational method will be developed to decrease testing time and provide an unbiased mathematical measurement of the response's validity. The joint use of signal-to-noise ratio estimation and cross correlation techniques will successfully reduce the number of sweeps necessary to obtain an VEP response and improve the 
current averaging method. The averaging process can be stopped sooner when the SNR pre-stimulus and post-stimulus portions can be compared and evaluated every 25 sweeps. A criterion can be developed to estimate the SNR for both pre-stimulus and post-stimulus portions and can successfully reduce the number of sweeps necessary to obtain an VEP response without reducing the quality of that response.

\subsection{Improvements}

A mechanical packaging for the LED-Goggle should be developed in the future. This new case should allow the clinician to adjust the stimulator's distance from the subject. The mechanical package will be similar to current virtual reality goggles. 


\section{APPENDIX}

\section{A.1 Calculation of size of the checks.}

The dimensions of each component or element can be determined by the visual angle that they subtend at the eye of the subject. Checks are expressed in minutes of arc and its visual angle can be calculated by the following formula:

$$
\mathrm{a}=\tan ^{-1}(\mathrm{~W} / 2 \mathrm{D}) \times 120
$$

where "a" is the visual angle in minutes of arc, "W" is the width of the check in millimeters and " $\mathrm{D}$ " is the distance of the pattern from the corneal surface in millimeters. Measurements of the visual angle in minutes or degrees can be converted to cycles per degree (cpd) by the following formula:

$$
\mathrm{cpd}=30 / \mathrm{W}
$$

where "W" is the diagonal measure of the check in minutes of arc. This measurement is defined as the spatial frequency of the stimulus (Celesia et al. 1993).

\section{A.2 Calculation of field size}

The field size of the stimulator can be described in terms of visual angle. Visual angle is commonly used to indicate the size of the pattern elements with shaper borders such as the square in checkerboard patterns. Visual angle can be calculated from

$$
\begin{aligned}
& \tan (\mathrm{B})=\mathrm{a} / \mathrm{b}, \\
& \text { so } \mathrm{B}=\tan ^{-1}(\mathrm{a} / \mathrm{b}),
\end{aligned}
$$

where "a" is the side length of the patterns and " $\mathrm{b}$ " is the distance from the eye. 


\section{A.3 Calculation of the Stimulus Luminance}

The luminance of the field is measured by a photometer and is expressed in candela per square meter $\left(\mathrm{cd} / \mathrm{m}^{2}\right)$. Note that $3.43 \mathrm{~cd} / \mathrm{m}^{2}$ is equal to 1 footLambert (fL) photometric unit. The mean luminance is measured at the center of the field and can be expressed for spatially mirror symmetric stimuli by the following formula:

$$
\left(\mathrm{L}_{\max }+\mathrm{L}_{\min }\right) / 2
$$

where $\mathrm{L}_{\max }$ and $\mathrm{L}_{\min }$ represent the maximum and minimum luminance value across the stimulus field (Celesia et al. 1993).

\section{A.4 Calculation of the Contrast}

Contrast is the difference in luminance between the bright and dark portion of the pattern and is expressed by the following formula:

$$
\mathrm{C}=\left[\left(\mathrm{L}_{\max }-\mathrm{L}_{\min }\right) /\left(\mathrm{L}_{\max }+\mathrm{L}_{\min }\right)\right] \times 100 \%
$$

where $\mathrm{C}$ is the contrast in percent; $\mathrm{L}_{\max }$ and $\mathrm{L}_{\min }$ are the maximum and minimum luminances of the pattern (Celesia et al. 1993). 


\section{Bibliography}

Aidley, D. J. 1989. The Physiology of Excitable Cells, pp. 402-407. 3rd. Ed., London: Cambridge University Press.

Andersson, T., Siden, A. 1994. Comparison of visual evoked potentials elicited by lightemitting diodes and TV monitor stimulation in patients with multiple sclerosis and potentially related conditions. Electroencephalogr. Clin. Neurophysiol. 92:473-479.

Arden, G. G., Bodis-Wollner, I., Halliday A. M. 1977. Visual Evoked Potentials in Man: New Developments, ed. J. E. Desmedt, pp. 3-15. Oxford: Clarendon Press.

Celesia, G. G., Bodis-Wollner, I., Chatrian, G. E., Harding G.F.A., Sokol, S., Spekeijse, H. 1993. Recommended standards for electroretinograms and visual evoked potentials. Report of an IFCN Committee. Electroencephalogr. Clin. Neurophysiol. 87:421-436.

Ciganek, L. 1961. The EEG response (evoked potential) to light stimulus in man. Electroencephalogr. Clin. Neurophysiol. 13:165-172.

Daly, D.D., Pedley, T. A. 1990. Current Practice of Clinical Electroencephalography, pp. 594-619. New York: Raven Press.

Delgado, R. E., Özdamar, Ö., Eilers R. B. 1993. On-line Auditory Brainstem Responses Signal-to-Noise Estimation for Response Detection, pp. 1-3. Poster presented at the IEEE Engineering in Medicine biology Society, 15th Annual International Conference in San Diego, California.

Epstein, C.M. 1979. True checkerboard pattern reversal with light-emitting diodes. Electroencephalogr. Clin. Neurophysiol. 47:611-613.

Evans, B. T., Binnie, C. D., Lloyd, D. S. 1974. A simple visual pattern stimulator. Electroenceph. clin. Neurophysiol. 37:403-406.

Jeffreys, D. 1977. Visual Evoked Potentials in Man: New Developments, ed. J. E. Desmedt, pp. 134-167. Oxford: Clarendon Press.

Halliday, A. M., Barrett, G., Halliday, E., Michael, W. F. 1977. Visual Evoked Potentials in Man: New Developments, ed. J. E. Desmedt, pp. 121-133. Oxford: Clarendon Press.

Moller, A. R., Sclabassi, R., Krieger, D. N. 1985. Evoked Potentials in Intraoperative Monitoring, pp. 88 - 93. Baltimore: Williams \& Wilkins Press. 
Özdamar, Ö., Delgado, R. E. 1996. Measurement of signal and noise characteristics in ongoing auditory brainstem response averaging, pp. 2-11. In press to Annals of Biomedical Engineering.

Pratt, H., Schacham, S., Bark, S. 1984. A pattern reversal stimulator using optical fibers. Electroencephalogr. Clin. Neurophysiol. 59:172-174.

Nilsson, B. Y. 1978. Visual Evoked Potential Responses in Multiple Sclerosis: comparison of two methods for pattern reversal. J. Neurol. Neurosurg. Psychiatry, 41:499-504.

Sokol, S. 1977. Visual Evoked Potentials in Man: New Developments, ed. J. E. Desmedt, pp. 410-417. Oxford: Clarendon Press.

Spehlmann, R., 1985. Evoked Potential Primer, pp. 1-134. Boston: ButterworthHeinermann Co.

Spekreijse, H., Estevez, 0., Reits D. 1977. Visual Evoked Potentials in Man: New Developments, ed. J. E. Desmedt, pp. 16-69. Oxford: Clarendon Press.

Stanley, W.D., Dougherty, G. R., Dougherty, R. 1984. Digital Signal Processing, pp. 303-314. 2nd. ed. Reston: Prentice Hall Co.

Taylor, M. J., McCullock, D. 1992. Visual Evoked Potential in infants and children. J. Clin. Neurophysiol. 3:357-372. 